\title{
Modeling Wakes and
}

Blockage in Large-Scale Wind Farms

Jessica M. I. Strickland 


\section{MODELING WAKES AND BLOCKAGE IN LARGE-SCALE WIND FARMS}

Jessica Marjorie Irene Strickland 


\section{Graduation committee:}

Prof. Dr. Jennifer L. Herek (Chair)

Prof. Dr. rer. nat. Detlef Lohse (Promoter)

Assoc. Prof. Dr. ir. Richard J. A. M. Stevens (Supervisor)

Prof. Dr. ir. Roberto Verzicco

Prof. Dr.-ing. Dr. rer. pol. Wilko Rohlfs

Prof. Dr. ir. Carlos Simão Ferreira

Assoc. Prof. Dr. Anna S. von der Heydt

\section{UNIVERSITY OF TWENTE.}

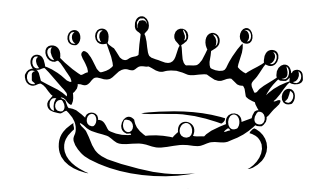

Physics of Fluids
University of Twente

University of Twente

University of Twente

University of Twente

University of Twente

Delft University of Technology

Utrecht University

The work in this thesis was carried out at the Physics of Fluids group of the Faculty of Science and Technology of the University of Twente. This thesis was financially supported by the Shell-NWO/FOM-initiative Computational sciences for energy research of Shell and Chemical Sciences, Earth and Live Sciences, Physical Sciences, FOM, and STW.

Dutch title:

HET MODELLEREN VAN WAKES EN BLOCKAGE IN GROTE WINDMOLENPARKEN

Publisher: Jessica M. I. Strickland, Physics of Fluids, University of Twente, P.O. Box 217, 7500 AE Enschede, The Netherlands

Cover: Design by the talented Dr. Luca Ricciardi. Front: Vertically staggered wind turbines. Back: A reflection of the turbines inspired by M.C. Escher's Three Worlds (1955).

ISBN: 978-90-365-5267-7

DOI: $10.3990 / 1.9789036552677$

๑ 2021 Jessica Marjorie Irene Strickland, The Netherlands. All rights reserved. No parts of this thesis may be reproduced, stored in a retrieval system or transmitted in any form or by any means without permission of the author. 


\section{MODELING WAKES AND BLOCKAGE IN LARGE-SCALE WIND FARMS}

\section{DISSERTATION}

to obtain

the degree of doctor at the Universiteit Twente, on the authority of the rector magnificus, prof. dr. ir. A. Veldkamp, on account of the decision of the Doctorate Board, to be publicly defended on Thursday the $2^{\text {nd }}$ of December, 2021, at 16:45 hours

by

Jessica Marjorie Irene Strickland

born on the $10^{\text {th }}$ of July, 1992

in Corner Brook, Newfoundland, CA. 
This dissertation has been approved by:

\section{Promoter}

Prof. dr. rer. nat. Detlef Lohse

\section{Co-Promoter}

Assoc. Prof. Dr. ir. Richard J. A. M. Stevens 
Dedicated to my mother,

to whom this thesis means so little and so much. 


\section{Contents}

Nomenclature ix

1 Introduction 1

1.1 General concept and motivation $\ldots \ldots \ldots \ldots \ldots \ldots$

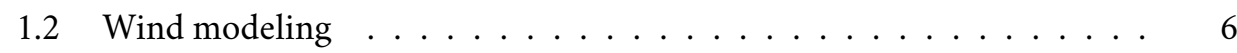

1.3 Downstream: Wakes . . . . . . . . . . . . . . . . . . . 9

1.4 Upstream: Induction and blockage $\ldots \ldots \ldots \ldots . \ldots \ldots$

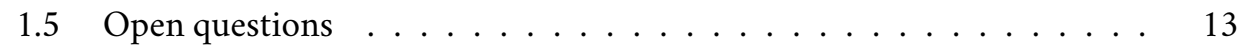

$1.6 \quad$ A guide through the thesis $\ldots \ldots \ldots \ldots \ldots \ldots$

\begin{tabular}{|l|l}
\hline I Vertically staggered wind farms & 17
\end{tabular}

2 Optimizing vertically staggered wind farms using an analytical model 19

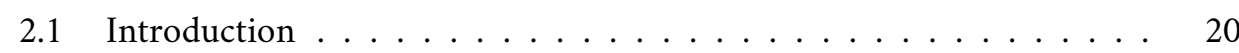

2.2 Model . . . . . . . . . . . . . . . . . . . . 22

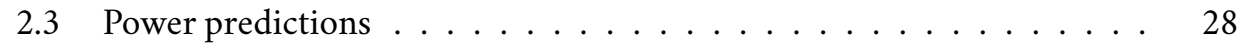

2.4 Optimizing inter-turbine spacing. . . . . . . . . . . . 34

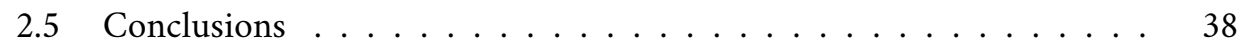

3 Large eddy simulations of vertically staggered wind farms 41

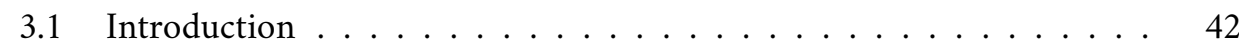

3.2 Numerical framework $\ldots \ldots \ldots \ldots \ldots$. . . . . . . . . . . . . . . . . . . . . . . . . . . . . .

3.3 Results and discussion . . . . . . . . . . . . . . . . . . . 51

3.4 Conclusions . . . . . . . . . . . . . . . . 60 
II Flow blockage in large-scale wind turbine arrays 63

4 Blockage effects in large wind turbine arrays in a neutral boundary layer 65

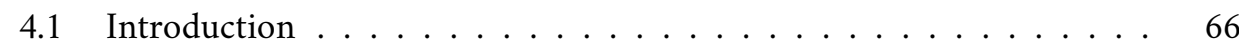

4.2 Numerical framework $\ldots \ldots \ldots$. . . . . . . . . . . . . . 68

4.3 Results and discussion $\ldots \ldots \ldots \ldots \ldots \ldots$. . . . . . . . . . . . . . . . . . . . .

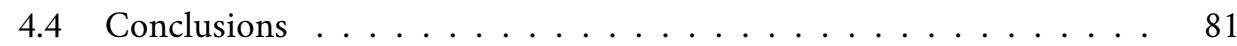

$5 \quad$ Effect of thrust coefficient on blockage in closely-spaced turbine arrays 83

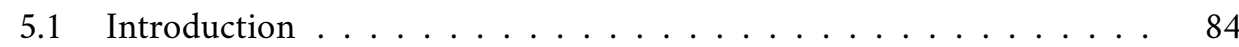

5.2 Numerical framework $\ldots \ldots \ldots \ldots \ldots$. . . . . . . . . . 85

5.3 Results and discussion $\ldots \ldots \ldots \ldots$

5.4 Conclusions . . . . . . . . . . . . . . . . . . 91

$6 \quad$ Wind farm blockage in a stable atmospheric boundary layer 93

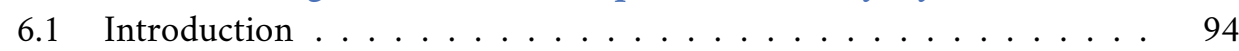

6.2 Numerical framework $\ldots \ldots \ldots \ldots$

6.3 Results and discussion . . . . . . . . . . . . . . . . . . . . 100

6.4 Conclusions . . . . . . . . . . . . . . . . . . . . . . . . 110

$\begin{array}{lll}7 & \text { Conclusions and outlook } & 111\end{array}$

$\begin{array}{ll}\text { Summary } & 121\end{array}$

$\begin{array}{ll}\text { Samenvatting } & 123\end{array}$

\begin{tabular}{ll}
\hline References & 127
\end{tabular}

\begin{tabular}{ll}
\hline Acknowledgements & 141
\end{tabular}

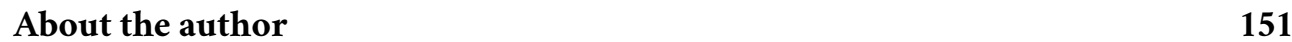




\section{Nomenclature}

\begin{tabular}{ll} 
Roman & \\
\hline Symbol & Description \\
\hline$a$ & Axial induction factor \\
$c^{f t}$ & Loading coefficient or planform thrust coefficient \\
$f_{c}$ & Coriolis parameter where $f_{c}=2 \Omega \sin \phi$ \\
$g$ & Acceleration due to gravity \\
$f_{i}$ & Turbine forcing \\
$p$ & Averaged pressure \\
$p^{*}$ & Modified pressure \\
$q$ & heat flux \\
$s$ & Dimensionless mean turbine spacing where $s=\sqrt{s_{x} s_{y}}$ \\
$s_{x}$ & Dimensionless streamwise turbine spacing where $s_{x}=S_{x} / D$ \\
$s_{y}$ & Dimensionless spanwise turbine spacing where $s_{y}=S_{y} / D$ \\
$u$ & Streamwise velocity along the $x$ direction \\
$u_{*}$ & Friction or shear velocity \\
$v$ & Spanwise velocity along the $y$ direction \\
$v_{\text {mag }}$ & wind magnitude where $v_{\text {mag }}=\sqrt{u^{2}+v^{2}}$ \\
$w$ & Vertical velocity along the $z$ direction \\
$z_{0}$ & Surface roughness scale \\
$z_{h}$ or $H_{T}$ & Turbine hub-height \\
\hline$A$ & Turbine's rotor-swept area where $A=\pi R^{2}$ \\
$C_{r}$ & Surface cooling rate \\
$C_{\text {smag }}$ & Smagorinsky coefficient \\
$C_{T}$ & Turbine thrust coefficient \\
$C_{T}^{\prime}$ & Disk-based turbine thrust coefficient \\
$D$ & Diameter of the turbine's rotor-swept area \\
$D_{\text {smag }}$ & Smagorinsky coefficient for sub-grid scale heat flux \\
& \\
\hline &
\end{tabular}


$F_{T} \quad$ Thrust force exerted by the turbines

$G \quad$ Geostrophic velocity magnitude

$H$ or $\delta \quad$ Height of the domain

$H_{\text {IBL }}$ or $\delta_{\text {IBL }} \quad$ Height of the internal boundary layer

$H_{T}$ or $z_{h} \quad$ Turbine hub-height

I Turbulence intensity

$L \quad$ Obukhov length

$L_{x} \quad$ Domain length in the $x$ direction

$L_{y} \quad$ Domain length in the $y$ direction

$L_{z} \quad$ Domain length in the $z$ direction

$N_{x} \quad$ Number of grid points in the $x$ direction

$N_{y} \quad$ Number of grid points in the $y$ direction

$N_{z} \quad$ Number of grid points in the $z$ direction

$N_{T} \quad$ Number of turbines

$N_{T, x} \quad$ Number of turbine rows

$N_{T, y} \quad$ Number of turbine columns

$R \quad$ Radius of the turbine rotor-swept

$P \quad$ Power

$S \quad$ Strain rate tensor

$S_{x} \quad$ Streamwise turbine spacing

$S_{y} \quad$ Spanwise turbine spacing

$U_{g} \quad$ Streamwise geostrophic velocity component

$U_{\infty} \quad$ Undisturbed upstream velocity

$V_{g} \quad$ Spanwise geostrophic velocity component 


\section{Greek}

\begin{tabular}{ll}
\hline Symbol & Description \\
\hline$\beta$ & Ch2 Linear cost coefficient, Ch 6 Buoyancy parameter \\
$\kappa$ & The von Kármán constant \\
$\delta$ or $H$ & Height of the domain \\
$\delta_{i j}$ & Kronecker Delta \\
$\delta_{\text {IBL }}$ or $H_{\text {IBL }}$ & Height of the internal boundary layer \\
$\nu$ & Wake eddy viscosity \\
$\nu_{T}$ & Turbulent eddy viscosity \\
$\nu_{\theta}$ & Eddy heat diffusivity \\
$\phi$ & Latitude, $(+)$ and (-) in the Northern and Southern hemisphere, respectively \\
$\psi$ & Stability corrections for momentum and heat flux \\
$\rho$ & Density of air \\
$\tau$ & Sub-grid scale stress \\
$\theta$ & Ch 2 Quadratic cost coefficient, Ch 6 Potential temperature \\
$\theta_{0}$ & Reference potential temperature \\
$\zeta$ & Revenue coefficient \\
\hline$\Delta$ & Resolved scale \\
$\Delta_{x}$ & Resolution in the $x$ direction where $\Delta_{x}=L_{x} / N_{x}$ \\
$\Delta_{y}$ & Resolution in the $y$ direction where $\Delta_{y}=L_{y} / N_{y}$ \\
$\Delta_{z}$ & Resolution in the $z$ direction where $\Delta_{z}=L_{z} / N_{z}$ \\
$\Omega$ & Rotation of the Earth \\
\hline
\end{tabular}




\section{Introduction

\section{1

\section{roduction} \\ (1)}

CHAPTER 1

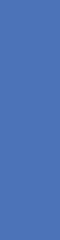

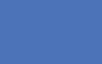

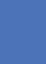

(

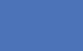

(1)

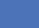

(

)

(

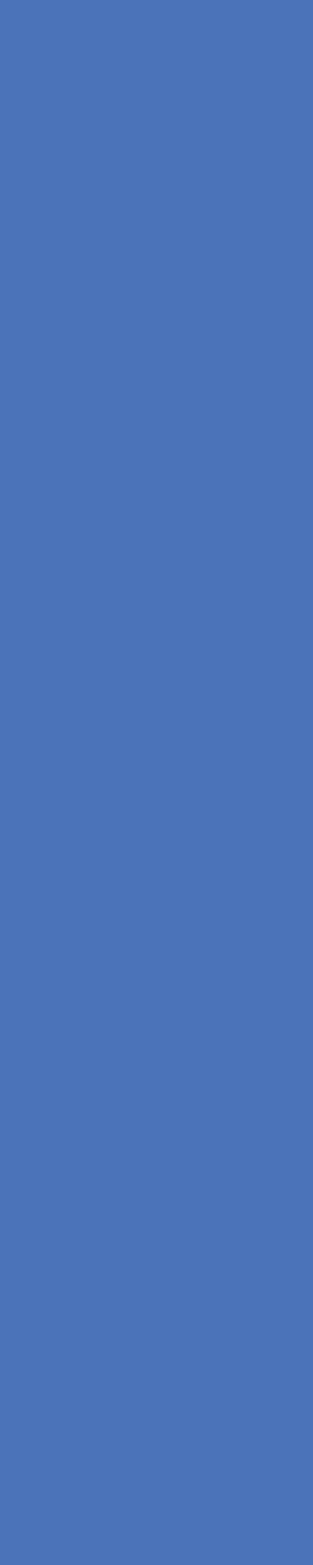




\subsection{General concept and motivation}

Turbulence in fluids is one of the oldest unsolved problems in classical physics according to the great Richard Feynman [1]. It has puzzled and delighted people for centuries, as you can see in Leonardo da Vinci's $16^{\text {th }}$ century sketch in Figure 1.1 We are exposed to turbulent, circulating fluid in everyday life, on many scales: the milk mixing into your coffee (or tea), the eddies dancing in a babbling brook, the wind that swirls around you, and so on. Understanding the physics of turbulence is as relevant as ever, especially regarding wind energy, in which turbulent physics determines the power output and longevity of wind farms. This thesis will shed light on the unsolved problem through the lens of wind farm productivity and performance.

Wind energy plays a vital role in mitigating the negative consequences of humandriven climate change. In today's environment, I should not have to lament about our predicament and explain why wind energy implementation, development, and research are both critical and urgent to achieve sustainability. But, of course, I will. The Earth is warming at an unprecedented rate as we continue to dump greenhouse gases into the atmosphere, in large part by consuming non-renewable energy resources. As we burn fossil fuels, the Earth burns with us, as global warming continues to cause irreversible damage [3]. In response, emerging industries for renewable energy sources, such as wind power, are expected to grow substantially in the coming decades. According to the Global Wind Energy Council, in order to avoid the worst impact of climate change, wind power growth must triple over the next decade alone [4]. However, harnessing wind energy for
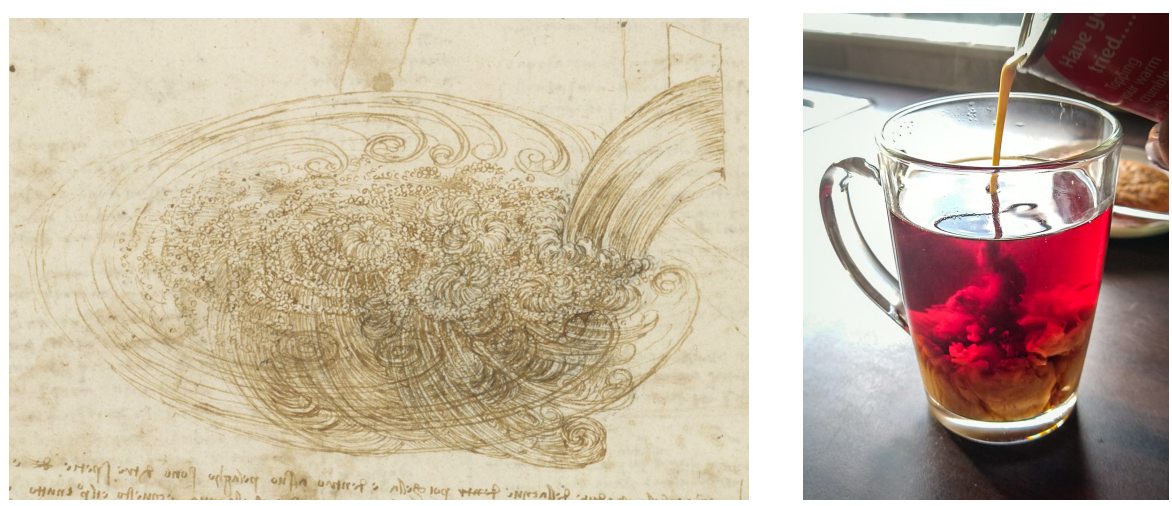

Figure 1.1: Left: Illustrated turbulent flow patterns exhibiting multiple scales of swirling eddies in water. Excerpt from Leonardo da Vinci's Studies of Water $(\sim 1510-1512$, Royal Collection Trust: RCIN 912660). Interesting details can be found in Reference 21. Right: Orange pekoe tea with evaporated milk mixing through turbulent motions driven by inertia and buoyancy. 

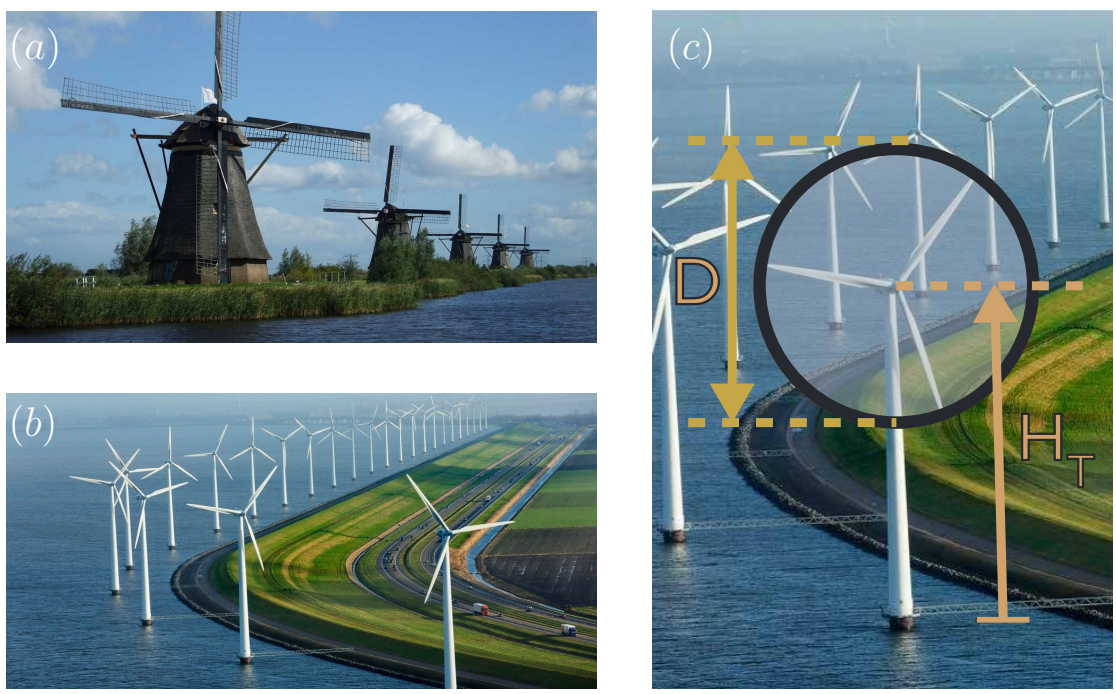

Figure 1.2: (a) Kinderdijk Windmolen in the Netherlands. Credit to www.theculturetrip.com. (b) First-generation Irene Vorrink wind farm. Credit to www.electrek.co. (c) A zoom-in of (b) indicating rotor diameter $D$ and hub-height $H_{T}$.

mechanical purposes is a long-established practice and dates back to ancient times [5-7]. In fact, you need not look farther than the Netherlands to discover a long history of wind power: from sail boats, grain-crushing water-pumping wind mills, to more modern electricity-generating wind turbines. Modern turbines convert the wind's kinetic energy into electrical power as an alternative to burning the depleting supply of fossil fuels. For example, in the Netherlands, they claim that all electric trains are now powered by wind energy [8]. Wind energy has come a long way and is being installed across the globe at an increasing capacity each year. To that end, more work is necessary and an obvious question persists: how can we optimize wind energy to produce as much power as possible?

We need to understand the fluid dynamics involved in order to optimize the performance of wind farms. Wind is a turbulent flow driven by atmospheric pressureand temperature-gradients. As this airflow passes through a wind turbine with a speed $u[\mathrm{~m} / \mathrm{s}]$, momentum is extracted and the kinetic energy $E[\mathrm{~J}]$ is converted into power $P[\mathrm{~W}]$ according to the following idealized equation which will be updated later $[9]$ :

$$
P \propto E / t=\frac{1}{2} m u^{2} / t=\frac{1}{2}(\rho A u) u^{2}=\frac{1}{2} \rho A u^{3}
$$

where $t[\mathrm{~s}]$ is time, $m[\mathrm{~kg}]$ is mass, $\rho\left[\mathrm{kg} / \mathrm{m}^{3}\right]$ is the density of air, and $A=\pi D^{2} / 4\left[\mathrm{~m}^{2}\right]$ is the rotor-swept area. The power generated by turbines will depend on multiple factors 


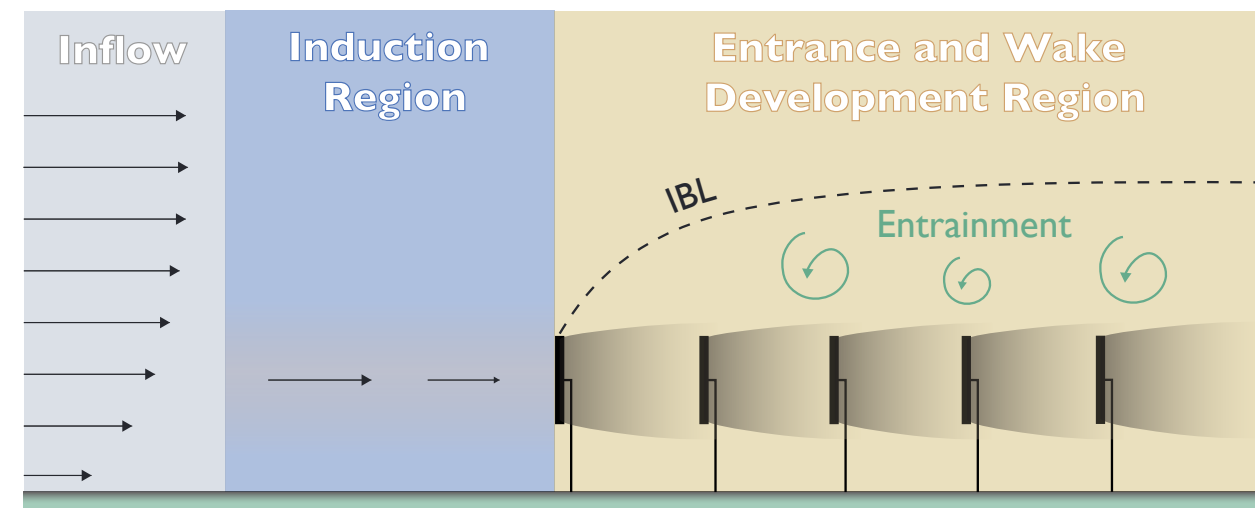

Figure 1.3: Sketch of the wind farm adjustment regions which are relevant to this research. The streamwise inflow is shown, followed by the upstream slowing of the incoming wind at hub-height in the induction region. An internal boundary layer (IBL) is formed by the wind farm at the entrance and develops downstream as wake production and recovery are balanced.

such as the turbine features, the terrain and layout, as well as the atmospheric conditions. The main challenge when predicting wind farm performance is understanding the turbulent multi-scale, two-way interactions with the atmosphere.

In this thesis, I focus on two physical phenomena affecting wind farm performance: wakes and blockage. Wind turbines reside in the atmospheric boundary layer (ABL), which is the lowest part of the atmosphere in contact with and influenced by the surface. Inherently, a turbine influences the ABL both downstream and upstream and the adjustment regions are shown in Figure 1.3 After air passes through a turbine, the downstream region is characterized by a velocity deficit that is even more turbulent, defined as a wake. Additionally, flow is affected in the induction region preceding the turbines. Wind turbines act as an obstruction, reducing the velocity upstream and deflecting flow, known as blockage. Essentially, wind farm performance depends on the relative positioning of turbines and how they interact with the atmosphere and each other. Modeling is an excellent method for better understanding these complex interactions and investigating wind farm productivity prior to construction. The work in this thesis uses modeling and simulations to investigate both wake and blockage effects in large-scale wind farms. Therefore, brief insight into modeling basics and parameters for a turbulent ABL are provided in the next section. Then, turbine wakes and blockage are explained and the relevant open questions that this thesis addresses are stated. Finally, a guide through the thesis is outlined at the end of this introduction. 


\subsection{Wind modeling}

In order to predict the performance of large-scale wind farms, we must understand the wind profile and the fluid physics driving it. Fluid models are a great tool for exploring turbulent behavior. The fidelity level of the preferred model depends on the desired information and accuracy as well as the required time, cost, and resources. In other words, some models are more detailed and computationally expensive, while others achieve sufficient insight and are less demanding by sacrificing accuracy with assumptions. In this section, relevant theory is described pertaining to the two types of modeling used in this thesis: a top-down analytical model and large eddy simulations. Chiefly, Figure 1.4 shows the normalized velocity profile of a neutral ABL (without thermal stratification or turbines) using both approaches. In general, the wind profile is influenced by the roughness of the surface and increases logarithmically as distance from the surface increases. As expected [10 11], The results of large eddy simulations (points) and the analytical model (lines) match quite well here.

(a)

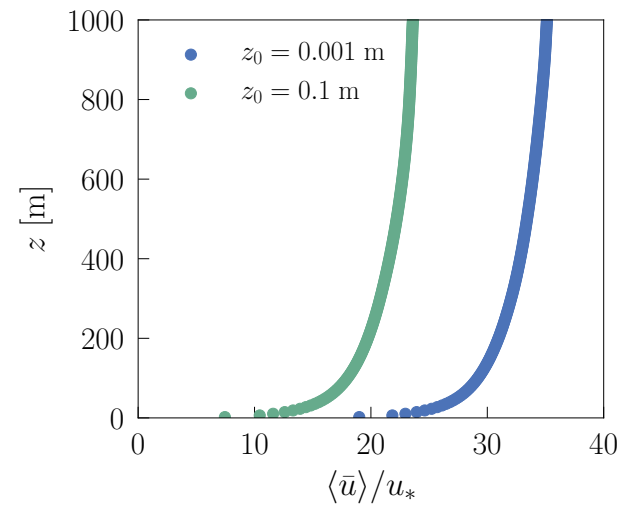

(b)

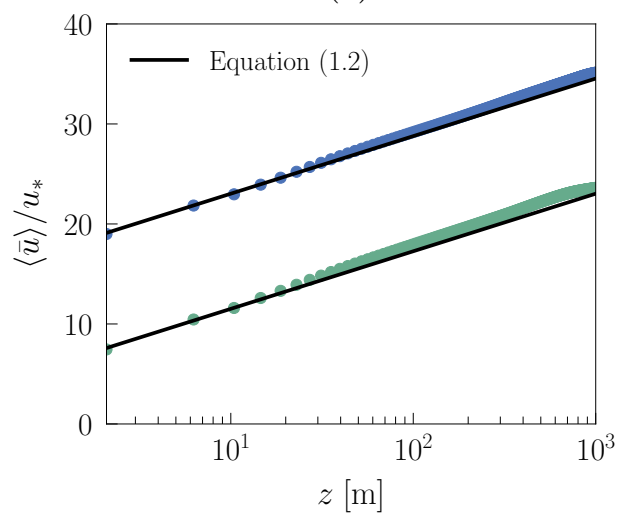

Figure 1.4: (a) Time- and horizontally-averaged streamwise velocity $u$ normalized by the friction velocity $u_{*}$ as a function of height $z$ for a neutral ABL using large eddy simulations. The roughness scales $z_{0}=0.1 \mathrm{~m}$ (green) and $z_{0}=0.001 \mathrm{~m}$ (blue) correspond to onshore and offshore conditions, respectively. (b) The same data compared to the logarithmic-law for turbulent rough-wall-bounded flow. Note that it is often common practice in wind farm literature to plot the distance from the surface on the horizontal axis. 


\subsubsection{Analytical top-down model}

Analytical modeling describes a closed-form mathematical solution of physical equations, typically derived from the conservation of flow properties. Top-down means that the system is represented without knowing the details of the driving forces. To make an analogy, the bottom-up approach would be like reading a list of ingredients and guessing what kind of cake would be made, while the top-down approach would be tasting the finished cake without knowing the ingredients. Regarding wind energy, bottom-up is modeling the turbine wake and how that influences the atmosphere [12-14], while topdown emulates the atmosphere without the turbulent details [15- 18]. I use the top-down approach, representing an ABL without wind turbines based on the classic logarithmiclaw for turbulent rough-wall-bounded flow (Equation 1.2). The average streamwise velocity profile $u(z)$ is obtained by integrating the velocity gradient of a constant stress layer as it relates to the shear momentum flux:

$$
\left(\kappa z u_{*}\right) \frac{d u(z)}{d z}=u_{*}^{2} \rightarrow u(z)=\frac{u_{*}}{\kappa} \ln \left(\frac{z}{z_{0}}\right)
$$

where $\kappa=0.4$ is the von Kármán constant, $u_{*}[\mathrm{~m} / \mathrm{s}]$ is the friction velocity which reflects the shear stress, and $z_{0}[\mathrm{~m}]$ is the surface roughness scale which represents the friction of the ground and affects the turbulent intensity of the atmosphere [19-21]. In this case, the roughness scale will characterize either onshore or offshore environments, corresponding to $z_{0}=0.1 \mathrm{~m}$ and $z_{0}=0.001 \mathrm{~m}$, respectively [22]. Examples for a neutral ABL without turbines are shown in Figure $1.4(\mathrm{~b})$. A common approach to introduce wind turbines is modeling them as an effective surface roughness within a wake layer [18, 23. The application of this equation to fully-developed wind farms is described in more detail in Chapter 2. The simplicity of this representation makes this type of modeling quick and inexpensive, which is favorable for elucidating basic trends in large-scale wind farms. Unfortunately, this approach is based on several assumptions and the accuracy of the results is less reliable than more complex models, i.e. those detailed in the following section. Nonetheless, in Figure $1.4(\mathrm{~b})$, the logarithmic law matches quite well with higher-fidelity numerical simulations which are described in the next section. 


\subsubsection{Numerical modeling: Navier-Stokes equations}

For more detail, one turns to the Navier-Stokes equations which are used to describe the motion of a viscous fluid based on the conservation of momentum (Equation 1.3], mass (Equation 1.4), and heat (Equation 1.5] [19, 21, 24]:

$$
\begin{aligned}
& \frac{\partial \boldsymbol{u}}{\partial t}+(\boldsymbol{u} \cdot \nabla) \boldsymbol{u}=-2 \Omega \times \boldsymbol{u}-\frac{1}{\rho} \nabla p+\nu \nabla^{2} u-g \beta\left(\theta-\theta_{0}\right) e_{z}+\boldsymbol{f}, \\
& \boldsymbol{\nabla} \cdot \boldsymbol{u}=0 \\
& \frac{\partial \theta}{\partial t}+(\boldsymbol{u} \cdot \nabla) \theta=q
\end{aligned}
$$

where $\boldsymbol{u}(x, y, t)[\mathrm{m} / \mathrm{s}]$ is the velocity, $\boldsymbol{\Omega}[\mathrm{rad} / \mathrm{s}]$ is the Earth's rotation rate, $p\left[\mathrm{~kg} / \mathrm{ms}^{2}\right]$ is the pressure, $\rho\left[\mathrm{kg} / \mathrm{m}^{3}\right]$ is the density of the fluid, $\nu\left[\mathrm{m}^{2} / \mathrm{s}\right]$ is the kinematic viscosity, $\boldsymbol{g}\left[\mathrm{m} / \mathrm{s}^{2}\right]$ is the gravitational acceleration, $\beta\left[\mathrm{K}^{-1}\right]$ is the buoyancy parameter, and $\theta-\theta_{0}[\mathrm{~K}]$ is the difference between the potential temperature of a point and a reference value. So, how the fluid motion changes in time $\left(\frac{\partial \boldsymbol{u}}{\partial t}\right)$ depends on the advection of the fluid, the Coriolis force, the pressure gradient, viscous forces, buoyancy, and an external force $f$ per unit mass. In this thesis, the latter term represents the wind turbines which can be modeled as disks that exert a thrust force on the flow as illustrated in Figure 1.5(a). Simplifications are made, including the Boussinesq approximation. The atmosphere is very turbulent which can be gauged using the dimensionless Reynolds number, $R e=U L / \nu$ where the flow speed $U[\mathrm{~m} / \mathrm{s}]$ and the characteristic length $L[\mathrm{~m}]$ are compared to the viscosity of the fluid. The atmosphere has a high $R e \sim \mathcal{O}\left(10^{7}\right)-\mathcal{O}\left(10^{9}\right)[25$ [26], and therefore, viscous forces in Equation 1.3 are often neglected. The continuity equation (Equation 1.4 reflects how the mass changes, but assuming that the fluid is incompressible and the density along a streamline remains constant $(d \rho / d t=0)$, the right side of the equation is zero. The conservation of energy (Equation 1.5 ) is characterized by changes in the thermal energy of the system which depends on $q[\mathrm{Km} / \mathrm{s}]$, the turbulent heat flux. These equations can be solved using different approaches depending on the required accuracy and available resources.

This complex system of equations can be solved numerically, resolving a wide range of time and length scales. When considering the spectrum of eddy sizes, sketched in Figure 1.5 b), different simulation techniques calculate or model different ranges. For example, the Navier-Stokes equations are solved for all spatial scales using direct numerical simulation (DNS). Therefore, the generated velocity field is detailed and time-dependent, but the process is computationally expensive and not feasible for large-scale wind farm studies. On the other hand, assumptions can be made such that all scales are modeled, i.e. through Reynolds averaged Navier-Stokes (RANS) equations, where the mean values are determined. With RANS, the process is faster and less demanding, but accuracy is reduced 
(a)

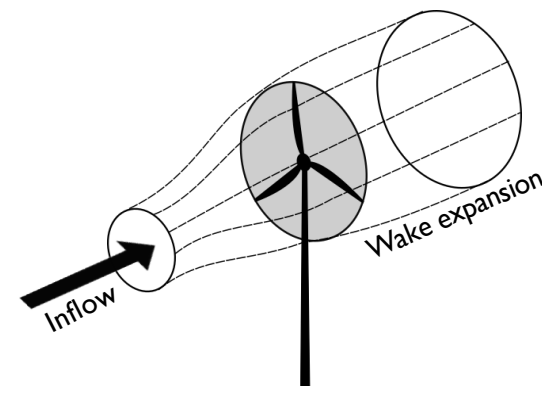

(b)

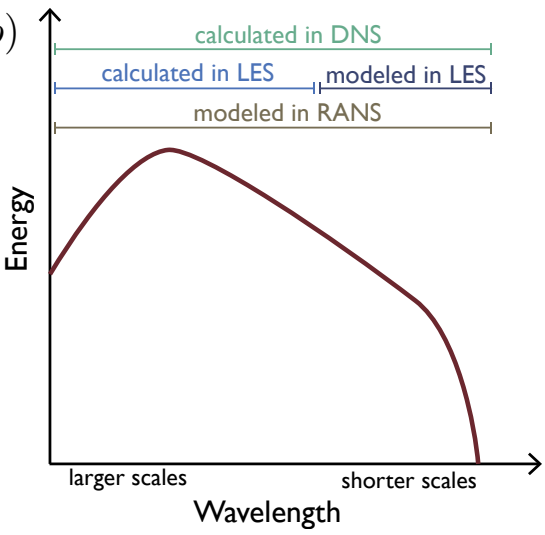

Figure 1.5: (a) A horizontal axis wind turbine can be represented by a disk (gray). (b) Sketch depicting the energy spectrum for isotropic turbulence within a range of wavelengths [19] accompanied by an indication of how ranges are handled by different numerical techniques: Direct numerical simulations (DNS), large eddy simulations (LES), and Reynolds averaged Navier-Stokes (RANS).

and time-dependent information is sacrificed. Throughout this work, I primarily use large eddy simulations which compromise some detail while still capturing the complex turbulent motions [27]. In the atmosphere, large-scale eddies are the most energetic [19. and the smaller scales are the most intensive to resolve. Therefore, large eddy simulations compute the Navier-Stokes equations for large length scales explicitly while filtering out and modeling the small scales using a sub-grid scale model [28 29]. In this manner, time-dependent velocity field data for large-scale wind farms can be obtained.

\subsection{Downstream: Wakes}

A wind turbine extracts momentum creating a wake downstream which is characterized by reduced velocity and increased turbulence. A wake expands laterally and vertically and then decays downstream due to turbulent diffusion 30. However, other turbines are often placed within the far-wake region of upstream turbines. Figure 1.6 is an infamous example visualizing turbine wakes as a low fog passes through the Horns Rev 1 wind farm, revealing the complex turbulent motions. These turbine trails are detrimental to downstream turbines, increasing their structural load due to increased turbulence while having a lower amount of kinetic energy available for extraction. Therefore, understanding wake interactions and how a wake recovers is necessary in order to optimize wind farm productivity while minimizing costs. 
(a)

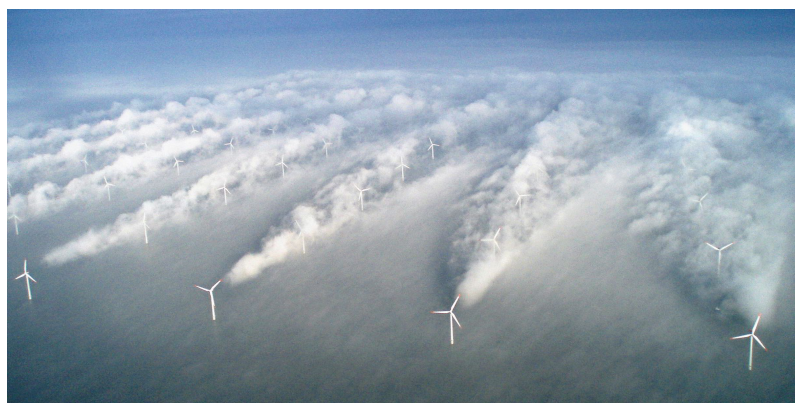

(b)

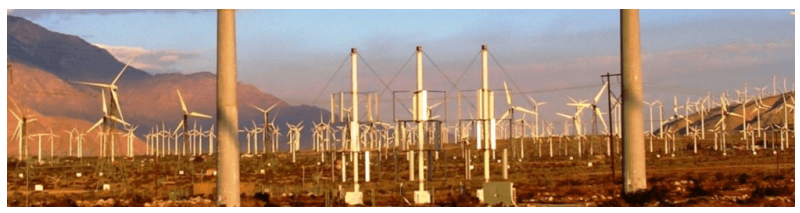

Figure 1.6: (a) Photograph of the Horns Rev 1 wind farm (12/02/2008 around 10:10 UTC). Photographer: Christian Steiness. (b) Short vertical axis turbines among tall horizontal axis turbines in San Gorgonio Wind Resource Area, California. Credit to: Windpower Engineering and Development.

Wind turbine wakes merge and the interaction between the wind farm and the lower part of the ABL forms an internal boundary layer (IBL) as illustrated in Figure $1.3,31,32$. The IBL develops at the entrance of the wind farm due to the sudden change in surface drag and the deceleration of the wind causes an upwards displacement of low-velocity flow. The IBL height reaches an asymptote downstream as a balance between wake production and recovery is achieved and above this height, the ABL is not disturbed by the turbines. The wind farm wake recovers via vertical turbulent motions replenishing the flow with higher velocity flow from above the wind farm, a process known as entrainment. If the distance between turbines is sufficient, the wake region is elongated and recovers before reaching the downstream turbines. However, this is often not the case. The first row of a wind farm will produce the most power because it is not subjected to any wakes. In contrast, subsequent turbine rows produce less power, even though an equilibrium is maintained between momentum extraction and higher velocity flow entrained from above [23]. Therefore, minimizing wake exposure will increase power production of the overall wind farm, but doing so by spreading the turbines out is not always feasible nor cost-effective.

Avoiding wind farm wake losses is imperative as the wind energy industry thrives, turbines become larger, and wind farms occupy an increasing amount of area. Interturbine wake interactions increase when the turbine size increases or when the spacing between them decreases. Turbine staggering is an innovative technique which can reduce 


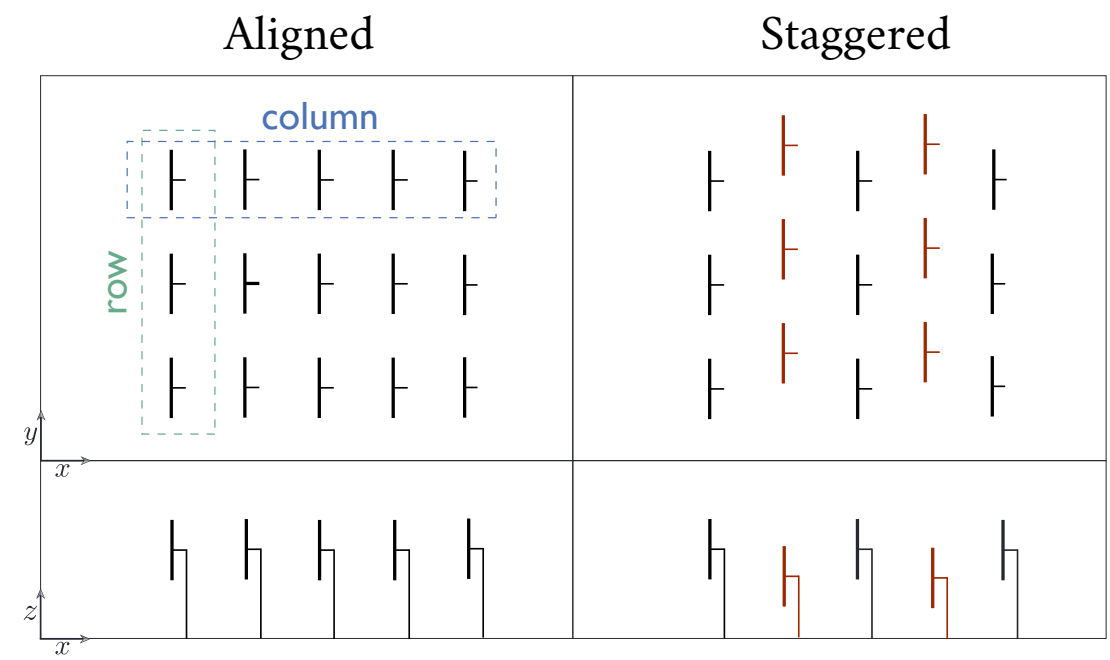

Figure 1.7: Sketch depicting an aligned wind farm $(l e f t)$ and a staggered wind farm (right). The top boxes are a top view showcasing horizontal staggering and the bottom boxes are a side view showcasing vertical staggering.

wake interactions without requiring more land area. This alternative involves misaligning the rotor areas, reducing overlap in either vertical or horizontal planes as illustrated in Figure 1.7 In this way, wake losses can be circumvented while taking advantage of relatively undisturbed flow. Horizontal staggering misaligns turbines with respect to the inflow direction so each consecutive turbine is not directly downstream of the preceding turbines. The benefits of this popular technique are well-established [33-36]. Vertical staggering involves adjusting the turbine height or diameter so that the turbine rotors do not wholly overlap in the vertical direction. Figure 1.6 b) shows an example of short vertical axis turbines placed among taller horizontal axis turbines. However, vertical staggering is relatively under-investigated, despite studies showing that this method can also increase turbine productivity [37-39]. Part I of this thesis focuses on the benefit of large-scale vertically staggered wind farms.

\subsection{Upstream: Induction and blockage}

The first row of a wind farm is often used as a reference under the assumption that it receives undisturbed flow. However, this is not the case because turbines influence the flow upstream. The wind slows down as it approaches a turbine, a phenomenon known as induction. In fact, this inherent velocity deficit preceding a single turbine at hub-height 


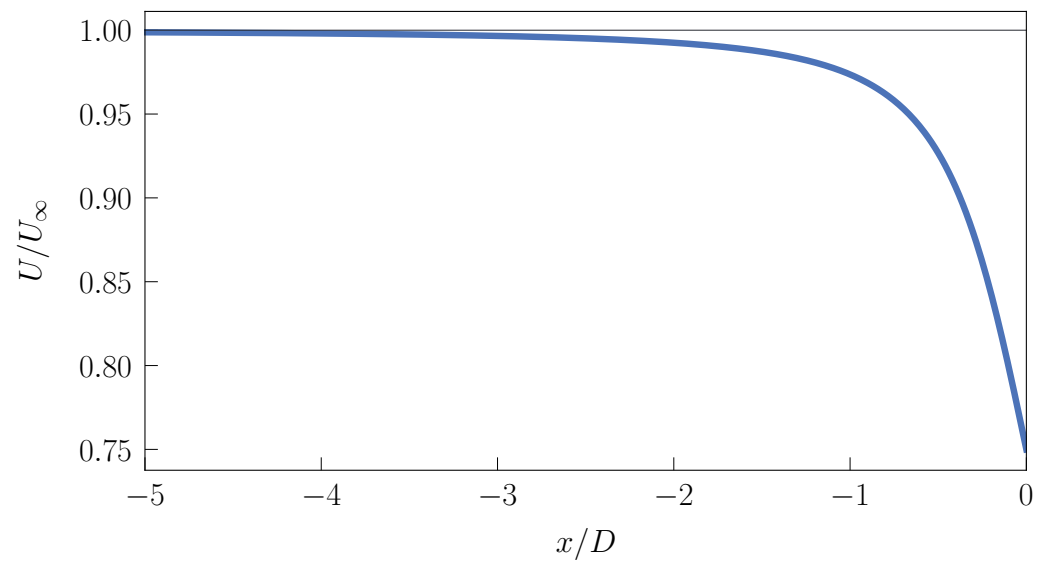

Figure 1.8: Streamwise centerline velocity preceding a wind turbine $(D=100 \mathrm{~m})$ placed at $x / D=0$, as described by Equation 1.6

is described by the Equation 1.6 obtained from actuator disk theory [40]. The velocity $U$ relative to the undisturbed upstream velocity $U_{\infty}$ along the symmetry axis is plotted in Figure 1.8

$$
\frac{U}{U_{\infty}}=1-a\left[1+\frac{x}{\left(x^{2}+R^{2}\right)^{1 / 2}}\right]
$$

where $x$ is the distance upstream, $R=D / 2$ is the rotor radius, and $a$ is the axial induction factor 40 41].

Wind not only slows down, but is deflected by the upcoming turbine, which I refer to as flow blockage. In this way, turbines can alter the kinetic energy available to neighboring turbines, ultimately influencing power production (refer to Equation 1.1). Therefore, the manner in which turbines are placed in both the spanwise and streamwise direction relative to each other will affect the airflow at the entrance of a wind farm. This phenomenon is only recently being given attention and is considerably less investigated compared to wake effects. As a result, blockage is relatively unaccounted for in wind farm planning. The impacts of blockage are subtler than wake losses, but nonetheless, interested parties are continuously striving for greater accuracy when predicting wind farm performance. Great strides have been made in the last few years, but a lack of consensus and several unanswered questions remain. Therefore, Part II of this thesis focuses on understanding flow blockage and the relevant parameters which affect power production. 


\subsection{Open questions}

- How effective is vertical staggering at mitigating wake losses and increasing profitability of large-scale wind farms? How are these results influenced by the spacing and relative position of the comparatively shorter turbines? Furthermore, how does the potential benefit of vertical staggering differ in either onshore or offshore conditions?

- What is the magnitude of flow blockage experienced by large-scale wind farms and how is blockage influenced by certain wind farm parameters? For instance, to what degree is flow blockage dependent on the turbine spacing, relative position, and thrust coefficient? Also, what role does thermal stratification of the atmosphere play in flow blockage effects?

\subsection{A guide through the thesis}

Optimizing power production in large-scale wind farms requires understanding the impacts of both wake and flow blockage effects. This thesis is divided into two parts devoted to each, addressing the open questions using fluid models. In Part I, I explore the potential benefit of vertically staggering wind turbines as an approach to mitigate wake losses. In Part II, I expand the relatively small knowledge base regarding flow blockage and the impact of this physical phenomenon on wind farm performance. A brief description of each topic is given below while a more detailed introduction prefaces each respective chapter.

\subsubsection{Part I: Vertically staggered wind farms}

Chapter 2 The benefits of vertically staggering wind turbines can be captured using an analytical model. Analytical models have been used extensively with the goal of optimizing wind farm performance. We develop on an analytical model for vertically staggered wind farms based on the logarithmic-law for turbulent rough-wall-bounded flow (Equation 1.2. This model is then used to explore the relative importance of different parameters, finding that the surface roughness as well as the turbine height, diameter, and spacing, greatly affect the vertically staggered wind farm's productivity. Thereafter, a simplified cost analysis reveals that adding short turbines to an array of tall turbines may not increase the profitability compared to a fixed array of only tall turbines. 
Chapter 3 Large eddy simulations are performed for a selection of vertically staggered wind farm configurations, inspired by the analytical model from Chapter 2 in order to provide more accurate results. In addition, a brief, but comprehensive introduction to the implementation of large eddy simulations is provided which is also applicable in Part II. We again find that adding short turbines to a fixed array of tall turbines increases the overall wind farm productivity, providing a greater benefit in offshore conditions versus onshore conditions. However, here we focus on the relative placement and proximity of the short turbines because this cannot be captured by the analytical model and is found to impact the relative benefit of vertical staggering.

\subsubsection{Part II: Flow blockage in large-scale wind turbine arrays}

Chapter 4 Wind farm flow blockage is quantified and explored in a neutral ABL using large eddy simulations to shed light on this relatively under-investigated phenomenon. A single turbine, a solitary row of turbines, and a wind farm with eight rows are compared in order to distinguish between streamwise and spanwise blockage effects. We determine that, due to the deflection of flow, spanwise neighbors can increase power production while downstream turbines hinder the productivity of the first row of a wind farm. Overall, blockage is enhanced when the turbine spacing is small, but is not dependent on the turbulence intensity at hub-height.

Chapter 5 Blockage in a neutral $\mathrm{ABL}$ is re-visited, determining how the blockage effect is dependent on the turbine thrust coefficient. Again, we compare the performance of a single turbine, a solitary row of turbines, and a wind farm with eight rows, but now while systematically varying the thrust coefficient. Trends emerge and we observe that the blockage effect is enhanced as the thrust coefficient increases because a higher thrust relates to more production and therefore more blockage by magnitude.

Chapter 6. We investigate how thermal stratification of the atmosphere influences streamwise flow blockage by comparing the first row of a wind farm to a solitary row of turbines. The previous chapters considered neutral ABL conditions, but here, we perform large eddy simulations with stable ABL conditions. The stability of the atmosphere is varied by cooling the surface at different rates which will affect flow motions within the ABL. We observe that blockage is enhanced when the atmosphere is stably stratified. 


\subsubsection{Conclusions and outlook}

Chapter 7: I summarize the findings of this research, propose potential improvements, and highlight unanswered questions. Additionally, I briefly explore topics which sparked interest but fell outside the scope of this thesis. These reflections are intended to inspire future research. 


\section{Vertically staggered wind farms \\ Verd}

Part I

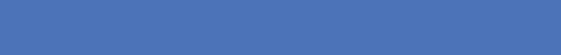

\section{Part I}

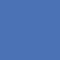





\section{Chapter 2: \\ OPTIMIZING VERTICALLY STAGGERED WIND FARMS USING AN ANALYTICAL MODEL}

Staggering wind turbines in the vertical direction can take advantage of undisturbed flow and circumvent wake losses. In this Chapter, we have adapted a top-down analytical model to investigate the potential benefit and profitability of vertically staggered wind farms taking into account the overall production as well as simplified costs. The productivity of the wind farm configuration depends on characteristics such as the roughness scale, turbine spacing, diameter, and hub-height, which can readily be adjusted and optimized using this analytical model. For instance, we observe that even though smaller turbines may avoid wake losses, when the short turbine diameter and height increase, they contribute more power due to their size. In other words, avoiding wake exposure at all costs is not necessarily optimal. Overall, we find that short turbines placed in an array of tall turbines increase the wind farm's total power production and that this benefit is greater in offshore conditions compared to onshore conditions. Nonetheless, power production should not be the sole consideration as we observe that increasing the number of short turbines does not produce a linear increase in power and these turbines also incur additional costs. Our simplified cost analysis suggests that placing short turbines in an array of tall turbines may not be more profitable than having only tall turbines.

To be submitted as: Jessica M. I. Strickland and Richard J. A. M. Stevens, Optimizing vertically staggered wind farms using an analytical model, Applied Energies. 


\subsection{Introduction}

Wind farms are becoming commonplace as the world grasps at renewable energy sources. These farms can take up costly land area and compete for space with sprawling populations and other developments. However, if turbines are arranged too closely, their wake interactions are known to negatively impact power production. One method to circumvent these losses is vertically staggering the turbines such that shorter turbines can take advantage of undisturbed airflow beneath taller turbines as illustrated in Figure 2.1 However, the potential benefit of these additional turbines will depend on multiple factors such as the turbine size and spacing. For instance, if the short turbines either have a smaller diameter, are placed more sparsely or closer to the ground, they will suffer less from wake interactions but their overall power contribution is less due to their small size, number, and the fact that wind near the surface has a lower velocity. Balancing these effects is required in order to optimize a multi-scale wind farm layout.

Modeling is a useful tool for investigating this balance and determining if a vertically staggered wind farm configuration can be advantageous prior to construction. Highfidelity large eddy simulations have shown that vertically staggered wind farms can be more productive than vertically aligned wind farms $39,42,43$. While these large eddy simulations are an excellent tool for studying turbulence, their high cost limits the variety of scenarios which can be explored. Wind tunnel experiments have supported these findings, also showing that vertically staggered wind farms can be more productive [44, 45]. Beyond productivity, other studies have approached the problem considering the costs of such layouts [46-55]. Although these studies use models that are less computationally expensive than large eddy simulations, many are bottom-up models which do not account

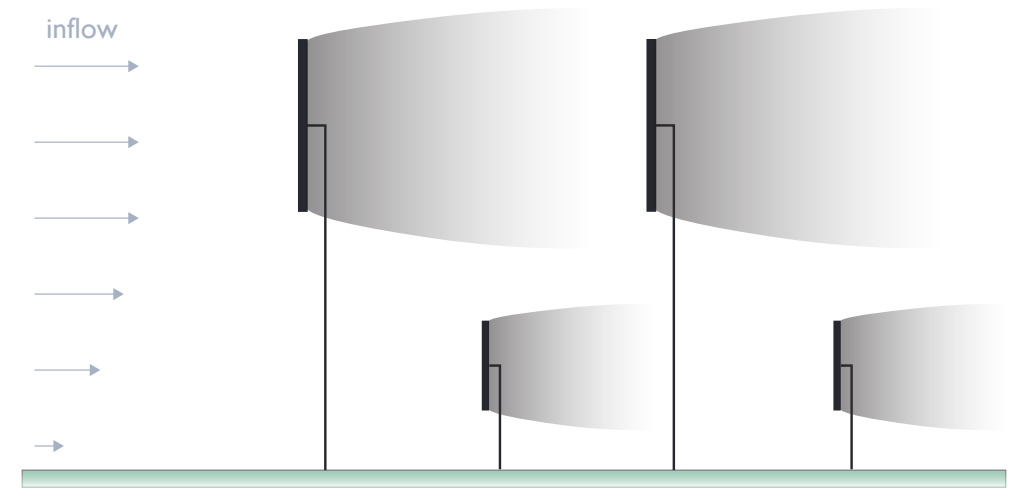

Figure 2.1: Sketch of how vertically staggered turbines can avoid wake losses and take advantage of undisturbed flow. 
for the large-scale interactions which are important when considering the influence of tall and short turbines. Furthermore, the majority of these studies present the results of very particular cases. Therefore, we aim to use a simple top-down model to investigate the importance of a variety of parameters which are detailed below.

The analytical model we use to study vertically staggered wind farms is based on a well-established top-down model which we have adapted to be more applicable and realistic. Initially, the top-down modeling approach was introduced by Frandsen et al. [16, 17] and has been further developed by Calaf et al. [18] to include wake effects [56]. The model has been developed further to incorporate physics which affect real wind farms. For instance, the neutrally-stratified atmospheric boundary layer $(\mathrm{ABL})$ is often capped by a stably-stratified free atmosphere which can affect the interaction between the ABL and large wind farms. With that in mind, Abkar and Porté-Agel [56] modified the Frandsen model to account for the effect of free-atmosphere stability. Later, Meneveau [31] extended the model to represent large but finite-size wind farms, with validation given by Stevens et al. [32]. Though still infinite, Xie et al. 37] extended the approach to consider vertically staggered wind farms with horizontal and vertical axis wind turbines in a neutral ABL. Altogether, we emphasize that this modeling approach has been extensively verified against large eddy simulation results in previous work for infinite arrays [18, large finite arrays 32 , and vertically staggered wind farms [37]. In fact, the agreement observed by Xie et al. 37] improves when a typo is amended, appearing in their Equations $(17,22)$. In the present work, we use this analytical model to investigate the potential benefit of vertical staggered wind farms, following and adapting the approach outlined by Xie et al. 37]. In contrast, we replace the vertical axis wind turbines by more conventional horizontal axis wind turbines and permit a gap between the tall and short turbines' rotor-swept area. Furthermore, we consider the effects of the internal boundary layer (IBL) generated by the wind farm. The complete approach is outlined in Section 2.2 for consistency and clarity.

We use this adapted analytical model to provide insight into how the combination of tall and short turbines interact. The simplicity of the model allows us to explore multiple parameters, such as the roughness scale, hub-height, rotor diameter, and turbine density (number of turbines per unit area). Unlike higher-fidelity modeling, this analytical model can be used to readily explore and optimize a plethora of wind farm configurations. We begin by investigating the influence of these parameters on the power production in Section 2.3 Next, in Section 2.4 we select cases of interest to examine the model's capability of addressing the potential profitability of vertically staggered wind farms. We focus on the turbine density, as sufficient spacing is crucial for reducing wake losses. Meyers and Meneveau [57] use a simple top-down analytical model to optimize the spacing of large wind farms based on relative power production and Stevens et al. [58] applies this simple model while also considering the profitability of such vertically aligned configurations. 
Although power production may increase when the turbine spacing increases, so do the accompanying costs. Therefore, we also consider both productivity and simplified costs when evaluating performance by examining the normalized power per unit cost as well as normalized profitability. Information such as this can provide useful insight into which cases may be of interest for more-costly, high-fidelity simulations as well as designing vertically staggered farms.

\subsection{Model}

\subsubsection{General aspects}

Here, we outline the top-down analytical model which we adapted from Xie et al. [37]. In brief, we generate the average streamwise velocity profile for six layers as indicated in Figure 2.2 (a). The profile is comprised of classic logarithmic layers $(1,3,5$, and 6$)$ and two wake layers $(2,4)$, for the small and the tall turbines, respectively. Layer 6 is above the wind farm's IBL height $\delta_{\mathrm{IBL}}$ where the ABL is assumed to be undisturbed. An example of the resultant velocity profile is shown in Figure 2.2 (b).

The horizontally averaged velocity in the non-wake layers is described by the wellestablished logarithmic law for a turbulent boundary layer with a rough-wall, which is obtained by vertically integrating the velocity gradient of a constant stress layer:

$$
\left(\kappa z u_{*}\right) \frac{d u(z)}{d z}=u_{*}^{2} \rightarrow u(z)=\frac{u_{*}}{\kappa} \ln \left(\frac{z}{z_{0}}\right) .
$$

The eddy viscosity $\left(\kappa z u_{*}\right)$ contains $\kappa=0.4$ the von Kármán constant, $u_{*}$ the friction velocity, and $z_{0}$ the surface roughness scale. Three possible wind farm effective surface roughness scales $\left(z_{0, \mathrm{lo}}, z_{0, \mathrm{mi}}, z_{0, \mathrm{hi}}\right)$ and three corresponding friction velocities $\left(u_{*, \mathrm{lo}}, u_{*, \mathrm{mi}}, u_{*, \mathrm{hi}}\right)$ are applied to the different regions as shown in Figure 2.2

The wake layer velocity profiles are expected to be flatter than the non-wake layers due to increased mixing. Therefore, we use a similar approach, but the wake layers are parameterized by adding a constant wake eddy viscosity $\nu$ :

$$
\left(\kappa z u_{*}+\nu\right) \frac{d u(z)}{d z}=u_{*}^{2}, \text { where } \nu=\sqrt{\frac{c^{f t}}{2}} u\left(H_{\text {hub }}\right) D .
$$

Regarding the wake eddy viscosity, the characteristic length scale is the turbine diameter $D$ and the characteristic velocity scale is $\sqrt{\frac{c^{f t}}{2}} u\left(H_{\text {hub }}\right)$ reflecting the momentum deficit imposed by the turbines. $H_{\text {hub }}$ is the hub-height of either the tall or short turbines. The 
$(a)$

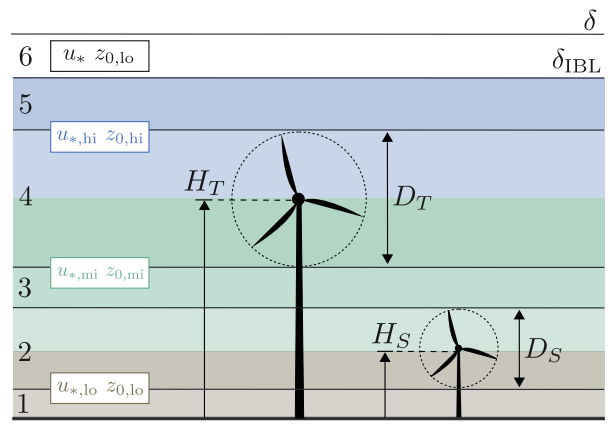

(b)

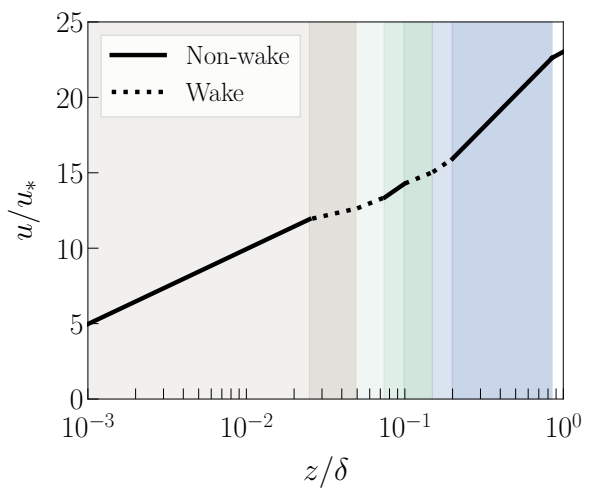

Figure 2.2: (a) Sketch of top-down model applied to a vertically staggered wind farm depicting six layers that are represented by different velocity profiles based on variables such as the boundary layer height $(\delta)$, turbine rotor diameters $\left(D_{T}, D_{S}\right)$, hub-heights $\left(H_{T}, H_{S}\right)$, friction velocity $\left(u_{*}\right)$, and roughness scale $\left(z_{0}\right)$. Regions 'low', 'middle', and 'high', are denoted by subscripts lo, mi, and hi, respectively. (b) The average velocity profile for a vertically staggered wind farm using model input listed in Table 2.1

required loading coefficient

$$
c^{f t}=\frac{C_{T} A}{S_{x} S_{y}}
$$

depends on the turbine thrust coefficient $C_{T}$, the rotor-swept area of the turbines $A=$ $\pi(D / 2)^{2}$, and the streamwise $S_{x}$ and spanwise $S_{y}$ inter-turbine spacing in meters. This loading coefficient also influences the momentum balance between the different layers and will be re-visited. Equation 2.2 is rewritten such that the wake eddy viscosity is transformed into the effective eddy viscosity $\nu^{*}$, and each wake layer is represented using two values above $\nu_{u}^{*}$ and below $\nu_{l}^{*}$ the hub-height of the turbines. As a result, the velocity profile of a wake layer can be obtained by integrating the following expression:

$$
\left(1+\nu^{*}\right) \frac{d u(z)}{d \ln \left(\frac{z}{H_{\text {hub }}}\right)}=\frac{u_{*}}{\kappa}, \text { where } \nu^{*}=\frac{\nu}{\kappa u_{*} z} .
$$




\subsubsection{The six velocity layer profiles}

Here we state the average streamwise velocity for each layer and then use these equations in combination with known physical relationships to derive the governing equations of the model. The turbine features and characteristics corresponding to each layer are displayed in Figure 2.2 a). Of these parameters, $C_{T}, D_{S}, D_{T}, H_{S}, H_{T}$, and $z_{0, \text { lo }}$ must be provided as model input, while $u_{*, i}, z_{0, \mathrm{mi}}, z_{0, \mathrm{hi}}$, and $\nu_{i}^{*}$ are calculated by the model. These variables are then used to generate the velocity profile, an example of which is shown in Figure 2.2 b). Based on Equations 2.1 and 2.4 the velocity profile for each layer is as follows:

1. Logarithmic layer between the surface and the lower edge of the short turbine rotor-swept area. This layer is characterized by the surface roughness scale $z_{0, \text { lo }}$ and the friction velocity $u_{*, \mathrm{lo}}$ :

$$
u(z)=\frac{u_{*, \mathrm{lo}}}{\kappa} \ln \left(\frac{z}{z_{0, \mathrm{lo}}}\right), \text { for } z_{0, \mathrm{lo}} \leqslant z \leqslant H_{S}-\frac{D_{S}}{2} .
$$

2. Wake layer corresponding to the rotor-swept area of the short turbines, parameterized using two effective eddy coefficients, which characterize the wake above $\nu_{S, u}^{*}$ and below $\nu_{S, l}^{*}$ the hub-height of the short turbines $H_{S}$ :

$$
\nu_{S, l}^{*}=\frac{\nu_{S}}{\kappa H_{S} u_{*, \mathrm{lo}}} \quad \nu_{S, u}^{*}=\frac{\nu_{S}}{\kappa H_{S} u_{*, \mathrm{mi}}} .
$$

The ratio between the effective eddy viscosity is thus given by

$$
\frac{\nu_{S, u}^{*}}{\nu_{S, l}^{*}}=\frac{u_{*, \mathrm{lo}}}{u_{*, \mathrm{mi}}}
$$

where the wake eddy viscosity is approximated as $\nu_{S}=\sqrt{\frac{c_{S}^{f t}}{2}} u\left(H_{S}\right) D_{S}$ and the loading coefficient of the short turbines is $c_{S}^{f t}=\frac{C_{T}^{S} A_{S}}{S_{x S} S_{y S}}$. Therefore, Equation 2.4 for this layer becomes

$$
\left(1+\nu_{S}^{*}\right) \frac{d u(z)}{d \ln \left(\frac{z}{H_{S}}\right)}=\frac{u_{*}}{\kappa}, \text { for } H_{S}-\frac{D_{S}}{2} \leqslant z \leqslant H_{S}+\frac{D_{S}}{2} .
$$

The velocity profile is obtained by vertically integrating and restricting the bound- 
ary by matching the mean velocity of the logarithmic layers at $z=H_{S} \pm \frac{D_{S}}{2}$ :

$$
\begin{array}{r}
u(z)=\frac{u_{*, \mathrm{lo}}}{\kappa} \ln \left[\left(\frac{z}{H_{S}}\right)^{\frac{1}{1+\nu_{S, l}^{*}}}\left(\frac{H_{S}}{z_{0, \mathrm{lo}}}\right)\left(\frac{H_{S}-\frac{D_{S}}{2}}{H_{S}}\right)^{\left.\frac{\nu_{S, l}^{*}}{1+\nu_{S, l}^{*}}\right],}\right. \\
\text { for } H_{S}-\frac{D_{S}}{2} \leqslant z \leqslant H_{S}, \\
u(z)=\frac{u_{*, \mathrm{mi}}}{\kappa} \ln \left[\left(\frac{z}{H_{S}}\right)^{\frac{1}{1+\nu_{S, u}^{*}}}\left(\frac{H_{S}}{z_{0, \mathrm{mi}}}\right)\left(\frac{H_{S}+\frac{D_{S}}{2}}{H_{S}}\right)^{\left.\frac{\nu_{S, u}^{*}}{1+\nu_{S, u}^{*}}\right],}\right. \\
\text { for } H_{S} \leqslant z \leqslant H_{S}+\frac{D_{S}}{2} .
\end{array}
$$

3. Logarithmic layer spanning the gap between the rotor-swept areas of the short and tall turbines which was outlined but not utilized by Xie et al. [37]. This layer is characterized by the roughness scale $z_{0 \text {,mi }}$ and the friction velocity $u_{*, \mathrm{mi}}$ :

$$
u(z)=\frac{u_{*, \mathrm{mi}}}{\kappa} \ln \left(\frac{z}{z_{0, \mathrm{mi}}}\right), \text { for } H_{S}+\frac{D_{S}}{2} \leqslant z \leqslant H_{T}-\frac{D_{T}}{2} .
$$

4. Wake layer corresponding to the rotor-swept area of the tall turbines, parameterized using two values of effective eddy viscosity below $\nu_{T, l}^{*}$ and above $\nu_{T, u}^{*}$ the hub-height of the tall turbines $H_{T}$ :

$$
\nu_{T, l}^{*}=\frac{\nu_{T}}{\kappa u_{*, \mathrm{mi}} H_{T}} \quad \nu_{T, u}^{*}=\frac{\nu_{T}}{\kappa u_{*, \mathrm{hi}} H_{T}} .
$$

The ratio between the effective eddy viscosity is thus given by

$$
\frac{\nu_{T, u}^{*}}{\nu_{T, l}^{*}}=\frac{u_{*, \mathrm{mi}}}{u_{*, \mathrm{hi}}}
$$

where $\nu_{T}=\sqrt{\frac{c_{T}^{f t}}{2}} u\left(H_{T}\right) D_{T}$ and $c_{T}^{f t}=\frac{C_{T}^{T} A_{T}}{S_{x T} S_{y T}}$, such that Equation 2.4 becomes

$$
\left(1+\nu_{T}^{*}\right) \frac{d u(z)}{d \ln \left(\frac{z}{H_{T}}\right)}=\frac{u_{*}}{\kappa}, \text { for } H_{T}-\frac{D_{T}}{2} \leqslant z \leqslant H_{T}+\frac{D_{T}}{2} .
$$


Similar to layer 2, this expression is integrated to arrive at the following velocity profile:

$$
\begin{array}{r}
u(z)=\frac{u_{*, \mathrm{mi}}}{\kappa} \ln \left[\left(\frac{z}{H_{T}}\right)^{\frac{1}{1+\nu_{T, l}^{*}}}\left(\frac{H_{T}}{z_{0, \mathrm{mi}}}\right)\left(\frac{H_{T}-\frac{D_{T}}{2}}{H_{T}}\right)^{\frac{\nu_{T, l}^{*}}{1+\nu_{T, l}^{*}}}\right], \\
\text { for } H_{T}-\frac{D_{T}}{2} \leqslant z \leqslant H_{T}, \\
u(z)=\frac{u_{*, \mathrm{hi}}}{\kappa} \ln \left[\left(\frac{z}{H_{T}}\right)^{\frac{1}{1+\nu_{T, u}^{*}}}\left(\frac{H_{T}}{z_{0, \mathrm{hi}}}\right)\left(\frac{H_{T}+\frac{D_{T}}{2}}{H_{S}}\right)^{\frac{\nu_{T, u}^{*}}{1+\nu_{T, u}^{*}}}\right], \\
\text { for } H_{T} \leqslant z \leqslant H_{T}+\frac{D_{T}}{2} .
\end{array}
$$

5. Logarithmic layer above the tall turbines but within the IBL. This layer is characterized by the roughness scale $z_{0, \mathrm{hi}}$ and the friction velocity $u_{*, \mathrm{hi}}$ :

$$
u(z)=\frac{u_{*, \mathrm{hi}}}{\kappa} \ln \left(\frac{z}{z_{0, \mathrm{hi}}}\right), \text { for } H_{T}+\frac{D_{T}}{2} \leqslant z \leqslant \delta_{\mathrm{IBL}} .
$$

6. Logarithmic layer above the IBL. This layer is characterized by the surface roughness scale $z_{0, \text { lo }}$ and the friction velocity $u_{*}$, reflective of the case without turbines:

$$
u(z)=\frac{u_{*}}{\kappa} \ln \left(\frac{z}{z_{0, \mathrm{lo}}}\right), \text { for } \delta_{\mathrm{IBL}} \leqslant z \leqslant \delta .
$$

Now we can use the streamwise velocity in combination with other relationships to close the system of equations and express the variables which are required to plot the velocity profile, i.e. the roughness scales $z_{0, \mathrm{mi}}, z_{0 \text {,hi. }}$. First, we consider the velocity at the hub-height of the short and tall turbines using the profile of the wake layer (Equations 2.92 .10 and 2.15 2.16:

$$
\begin{array}{r}
u\left(H_{S}\right)=\frac{u_{*, \mathrm{lo}}}{\kappa} \ln \left[\left(\frac{H_{S}}{z_{0, \mathrm{lo}}}\right)\left(\frac{H_{S}-\frac{D_{S}}{2}}{H_{S}}\right)^{\frac{\nu_{S, l}^{*}}{1+\nu_{S, l}^{*}}}\right] \\
=\frac{u_{*, \mathrm{mi}}}{\kappa} \ln \left[\left(\frac{H_{S}}{z_{0, \mathrm{mi}}}\right)\left(\frac{H_{S}+\frac{D_{S}}{2}}{H_{S}}\right)^{\frac{\nu_{S, u}^{*}}{1+\nu_{S, u}^{*}}}\right],
\end{array}
$$




$$
\begin{aligned}
u\left(H_{T}\right) & =\frac{u_{*, \mathrm{mi}}}{\kappa} \ln \left[\left(\frac{H_{T}}{z_{0, \mathrm{mi}}}\right)\left(\frac{H_{T}-\frac{D_{T}}{2}}{H_{T}}\right)^{\frac{\nu_{T, l}^{*}}{1+\nu_{T, l}^{*}}}\right] \\
& =\frac{u_{*, \mathrm{hi}}}{\kappa} \ln \left[\left(\frac{H_{T}}{z_{0, \mathrm{hi}}}\right)\left(\frac{H_{T}+\frac{D_{T}}{2}}{H_{S}}\right)^{\frac{\nu_{T, u}^{*}}{1+\nu_{T, u}^{*}}}\right] .
\end{aligned}
$$

The ratios corresponding to each equation are rearranged to express the relationship between the friction velocities:

$$
\begin{gathered}
\frac{u_{*, \mathrm{mi}}}{u_{*, \mathrm{lo}}}=\frac{\ln \left(\frac{H_{S}}{z_{0, \mathrm{lo}}}\right)+\frac{\nu_{S, l}^{*}}{1+\nu_{S, l}^{*}} \ln \left(\frac{H_{S}-\frac{D_{S}}{2}}{H_{S}}\right)}{\ln \left(\frac{H_{S}}{z_{0, \mathrm{mi}}}\right)+\frac{\nu_{S, u}^{*}}{1+\nu_{S, u}^{*}} \ln \left(\frac{H_{S}+\frac{D_{S}}{2}}{H_{S}}\right)}, \\
\frac{u_{*, \mathrm{hi}}}{u_{*, \mathrm{mi}}}=\frac{\ln \left(\frac{H_{T}}{z_{0, \mathrm{mi}}}\right)+\frac{\nu_{T, l}^{*}}{1+\nu_{T, l}^{*}} \ln \left(\frac{H_{S}-\frac{D_{T}}{2}}{H_{T}}\right)}{\ln \left(\frac{H_{S}}{z_{0, \mathrm{hi}}}\right)+\frac{\nu_{S, u}^{*}}{1+\nu_{T, u}^{*}} \ln \left(\frac{H_{T}+\frac{D_{T}}{2}}{H_{T}}\right)} .
\end{gathered}
$$

The friction velocity $u_{* \text {,hi }}$ is also restricted by the condition that the logarithmic layer must be continuous with the unperturbed velocity at the edge of the IBL such that

$$
u_{*, \mathrm{hi}}=u_{*} \frac{\ln \left(\delta_{\mathrm{IBL}} / z_{0, \mathrm{lo}}\right)}{\ln \left(\delta_{\mathrm{IBL}} / z_{0, \mathrm{hi}}\right)} .
$$

Next, the relationships between the momentum layers are expressed using the horizontally averaged momentum balance equations considering that turbine thrust causes momentum loss in the wake region:

$$
\begin{aligned}
& u_{*, \mathrm{mi}}^{2}=u_{*, \mathrm{lo}}^{2}+\frac{1}{2} c_{S}^{f t} u\left(H_{S}\right)^{2}, \\
& u_{*, \mathrm{hi}}^{2}=u_{*, \mathrm{mi}}^{2}+\frac{1}{2} c_{T}^{f t} u\left(H_{T}\right)^{2} .
\end{aligned}
$$

Finally, by substituting Equations 2.21 and 2.22 into the 2.24 and 2.25, respectively, the roughness scales can be expressed as follows making our list a closed system of equations which can be solved iteratively:

$$
\begin{array}{r}
z_{0, \mathrm{mi}}=H_{S}\left(\frac{H_{S}+\frac{D_{S}}{2}}{H_{S}}\right)^{\frac{\nu_{S, u}^{*}}{1+\nu_{S, u}^{*}}} \times \\
\exp \left(-\left[\frac{c_{S}^{f t}}{2 \kappa^{2}}+\left\{\ln \left[\frac{H_{S}}{z_{0, \mathrm{lo}}}\left(\frac{H_{S}-\frac{D_{S}}{2}}{H_{S}}\right)^{\left.\left.\frac{\nu_{S, l}^{*}}{1+\nu_{S, l}^{*}}\right]\right\}^{-2}}\right]^{-\frac{1}{2}}\right),\right.\right.
\end{array}
$$




$$
\begin{array}{r}
z_{0, \mathrm{hi}}=H_{T}\left(\frac{H_{T}+\frac{D_{T}}{2}}{H_{T}}\right)^{\frac{\nu_{T, u}^{*}}{1+\nu_{T, u}^{*}}} \times \\
\exp \left(-\left[\frac{c_{T}^{f t}}{2 \kappa^{2}}+\left\{\ln \left[\frac{H_{T}}{z_{0, \mathrm{mi}}}\left(\frac{H_{T}-\frac{D_{T}}{2}}{H_{T}}\right)^{\left.\left.\frac{\nu_{T, l}^{*}}{1+\nu_{T, l}^{*}}\right]\right\}^{-2}}\right]^{-\frac{1}{2}}\right) .\right.\right.
\end{array}
$$

\subsection{Power predictions}

This simple model allows us to readily explore multiple wind farm parameters and the potential benefits of vertically staggered wind farms. First, we compare the productivity of multiple vertically staggered wind farms to a reference case without short turbines, investigating the relative importance of the short turbine hub-height and diameter, the roughness scale, and the short turbine density. Then, we focus on the short turbine spacing, comparing the power output of vertically staggered turbines to an ideal free-standing turbine.

\subsubsection{Vertically aligned wind farm as a control case}

First, we quantify the power production of vertically staggered wind farms. The average power output of the tall $P_{T}$ and short turbines $P_{S}$, is proportional to the velocity at hub-height cubed:

$$
P_{T, S} \propto u\left(H_{T, S}\right)^{3}
$$

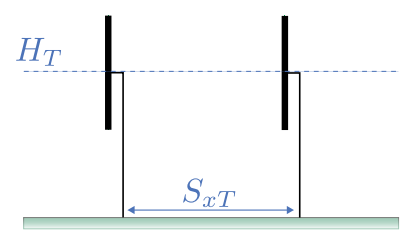

(a)

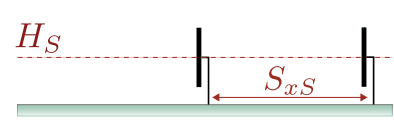

(b)

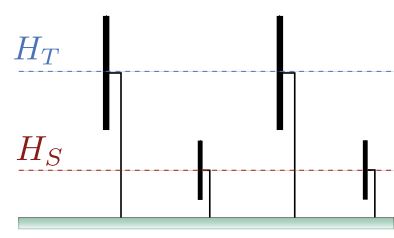

(c)

Figure 2.3: Schematic of (a) a tall vertically aligned wind farm, (b) a short vertically aligned wind farm, and (c) a vertically staggered wind farm in which both the vertically aligned configurations are combined. 
The velocity at hub-height for any particular setup is calculated using the model output and Equations 2.19 and 2.20. Also, the relative power of the short turbines is scaled by the difference in the thrust coefficient and rotor-swept area $\left(A \propto D^{2}\right)$. We note that although the model allows for different values of thrust coefficient for short and tall turbines, we proceed assuming that they are the same value $C_{T}$.

We explore the influence of the roughness scale $z_{0 \text {,lo }}$, the short turbine hub-height $H_{T}$, rotor diameter $D_{T}$, and relative number $N_{S}$, on the wind farm power output. The input parameters which remain constant throughout the study are summarized in Table 2.1 We begin by comparing the average power output of tall and short turbines in the vertically staggered wind farm $\left(P_{\text {total }}=P_{T}+N_{S} P_{S}\right.$, Figure 2.3 (c) $)$ to the average power of a tall turbine in a control case without short turbines $\left(P_{\text {control }}\right.$, Figure $\left.2.3(\mathrm{a})\right)$. The power output ratio is calculated while simultaneously varying the short turbine hub-height and diameter for three different scenarios presented in Figure 2.4

In Figure 2.4 (a), the tall and short turbines have the same spacing $s^{2}$ and therefore the ratio between the number of tall and short turbines is $N_{T}: N_{S}=1: 1$. The roughness scale is $z_{0,1 \mathrm{o}} / \delta=10^{-6}$, corresponding to offshore conditions [59]. Overall, we observe that the combined power output in the vertically staggered wind farm is higher than that of the control case with only tall turbines. Therefore, although the tall turbines may suffer due to the increased wake and kinetic energy extraction caused by the short turbines, the additional power provided by the short turbines makes the vertically staggered layout more productive. We observe that the relative productivity is highly dependent on the short turbine hub-height and diameter. The model results suggest that

\begin{tabular}{ll}
\hline Variable & Input \\
\hline$C_{T}$ & 0.84 \\
$\delta$ & $1000 \mathrm{~m}$ \\
$\delta_{\mathrm{IBL}}$ & $850 \mathrm{~m}$ \\
$z_{0, \mathrm{lo}}$ & $0.1 \mathrm{~m}$ \\
$H_{T}$ & $150 \mathrm{~m}$ \\
$D_{T}$ & $100 \mathrm{~m}$ \\
$H_{S}$ & $50 \mathrm{~m}$ \\
$D_{S}$ & $50 \mathrm{~m}$ \\
$s^{2}$ & $7.85 \times 5.24$ \\
$\beta$ & $5 \times 10^{-3}$ \\
\hline
\end{tabular}

Table 2.1: Vertically staggered wind farm constants unless otherwise specified. Each variable is defined in a symbols list provided after the table of contents on page $i x$. 
(a) Offshore, $N_{T}: N_{S}=1: 1$

$\mathrm{P}_{\text {total }} / \mathrm{P}_{\text {control }}$

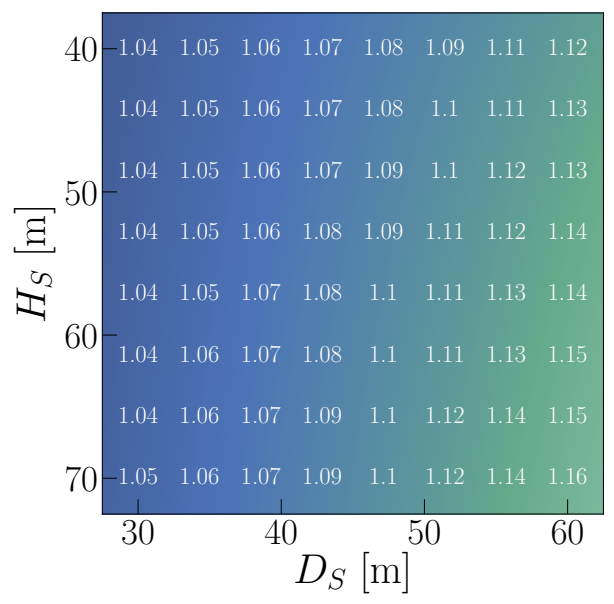

(b) Onshore, $N_{T}: N_{S}=1: 1$

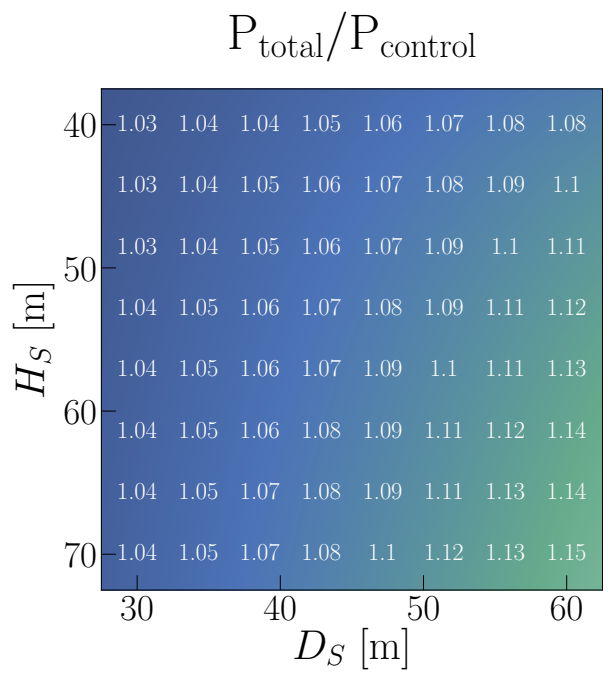

(c) Onshore, $N_{T}: N_{S}=1: 4$

$\mathrm{P}_{\text {total }} / \mathrm{P}_{\text {control }}$

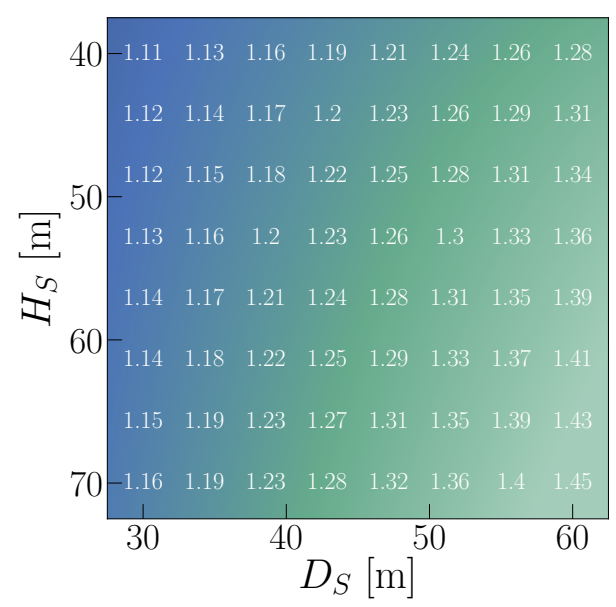

Figure 2.4: Average power output of turbines in a vertically staggered wind farm (Figure 2.3(c)) compared to the control case without short turbines (Figure 2.3 a)) for offshore and onshore conditions corresponding to $z_{0, \mathrm{lo}} / \delta=10^{-6}$ and $z_{0, \mathrm{lo}} / \delta=10^{-4}$, respectively. 
low ranges of hub-height and rotor diameter are not largely advantageous, increasing the relative power by $<5 \%$. In fact, increasing the height and diameter of the short turbines appears to be generally more productive. Essentially, although decreasing the size of the short turbines reduces overlap with tall turbines, and ergo wake interactions, smaller and shorter turbines ultimately generate and contribute less power.

In Figure 2.4(b), we maintain the turbine ratio $N_{T}: N_{S}=1: 1$, but the roughness scale is changed to $z_{0, \mathrm{lo}} / \delta=10^{-4}$, corresponding to onshore conditions. Comparing Figures 2.4(a) and 2.4(b), we observe that though the difference is subtle, the offshore case appears to be more impacted by vertical staggering, with slightly higher values across the board. Vasel-Be-Hagh and Archer [52] also observed a maximum benefit in offshore conditions using their modeling approach. Furthermore, we observe changes in the general trend with hub-height and diameter. In the onshore case, the short turbine hub-height appears to be more influential than in the offshore case where adjusting the diameter dominates changes in relative productivity. The ability to estimate in which conditions these parameters become most influential is a useful tool. In the remainder of the paper, we use $z_{0, \mathrm{lo}} / \delta=10^{-4}$ because we later apply a simplified cost analysis which is more applicable to onshore conditions.

Figure 2.4(c) is comparable to 2.4(b) where the roughness scale remains the same, but here the spacing between the short turbines is reduced such that there are four short turbines per tall turbine $N_{T}: N_{S}=1: 4$. We observe that incorporating more short turbines appears to be even more productive compared to the control case, with increased values across the board in Figure 2.4(c). However, this relative power increase is not linear with the number of turbines, implying that the turbines will suffer more when there are more short turbines present because the overall turbine density, and thus amount of wake interaction, increases. Therefore, the question arises: Does the increase in performance outweigh the costs and at what turbine spacing is this optimized? For simplicity, the remainder of the results use constant values of hub-height and diameter as the goal of the research is not to show a multitude of combinations but to explore the capability of using the simple model to determine the potential advantage of vertically staggered wind farms. Additionally, although we showed that larger values for the short turbine diameter and hub-height are beneficial, we select $D_{S}=50 \mathrm{~m}, H_{S}=50 \mathrm{~m}$, in order to study cases with minimal overlap between the wake of tall and short turbines. 


\subsubsection{Free-standing tall turbine as a control case}

We investigate the effect of spacing on the power output by partially following the analysis of Meyers and Meneveau [57] and Stevens et al. [32 58]. The average power output of a turbine in a wind farm $P \propto u\left(H_{T, S}\right)^{3}$ is normalized by the power output expected without wake interactions using the velocity at hub-height of a free-standing tall turbine $P_{T, 0} \propto u_{0}\left(H_{T}\right)^{3}$. Again, the relative power of the short turbines is scaled by the difference in the rotor-swept area. Therefore, the normalized power output of tall and short turbines can be expressed as follows:

$$
\begin{aligned}
& \frac{P_{T}}{P_{T, 0}}=\frac{u\left(H_{T}\right)^{3}}{u_{0}\left(H_{T}\right)^{3}}= \\
& \left(\frac{u_{\mathrm{hi}}^{*}}{\kappa} \ln \left[\left(\frac{H_{T}}{z_{0, \mathrm{hi}}}\right)\left(1+\frac{D_{T}}{2 H_{T}}\right)^{\frac{\nu_{T, u}^{*}}{1+\nu_{T, u}^{*}}}\right]\right)^{3} /\left(\frac{u^{*}}{\kappa} \ln \left(\frac{H_{T}}{z_{0, \mathrm{lo}}}\right)\right)^{3}, \\
& \frac{P_{S}}{P_{T, 0}}=\frac{A_{S}}{A_{T}} \frac{u\left(H_{S}\right)^{3}}{u_{0}\left(H_{T}\right)^{3}}= \\
& \frac{D_{S}^{2}}{D_{T}^{2}}\left(\frac{u_{\mathrm{mi}}^{*}}{\kappa} \ln \left[\left(\frac{H_{S}}{z_{0, \mathrm{mi}}}\right)\left(1+\frac{D_{S}}{2 H_{S}}\right)^{\frac{\nu_{S, u}^{*}}{1+\nu_{S, u}^{*}}}\right]\right)^{3} /\left(\frac{u^{*}}{\kappa} \ln \left(\frac{H_{T}}{z_{0, \mathrm{lo}}}\right)\right)^{3} .
\end{aligned}
$$

Figure 2.5 (a) shows the relative power as a function of dimensionless turbine spacing $\left(s_{x}=S_{x} / D, s_{y}=S_{y} / D\right)$ for vertically aligned wind farms with either only tall turbines or only short turbines as depicted in Figures 2.3 a) and (b), respectively. We observe that for both cases, the relative power increases as the turbine spacing increases, which is due to reduced wake interactions. As expected, the larger vertically aligned turbines have a higher relative power than the short vertically aligned turbines. Moreover, the relative power of the tall turbines approaches a value of one when the spacing is very large because the turbines will perform the same as free-standing tall turbines when spaced sufficiently apart. Meanwhile, the short turbines do not approach the same limit due to the fact that the power output is normalized by a free-standing tall turbine. This model was validated by comparing vertically aligned turbines to real wind farms in references [32, 57].

We extend this analysis to vertically staggered wind farms (Figure 2.3 (c)). In Figure 2.5 (b), the relative power is shown for a vertically staggered wind farm where the tall turbine spacing remains constant and the short turbine spacing is varied. These results are presented alongside the corresponding vertically aligned scenarios where only short turbine spacing is varied. Most importantly, we observe that the turbines within the vertically staggered farm are influenced by the large-scale interactions, a result that can 
(a)

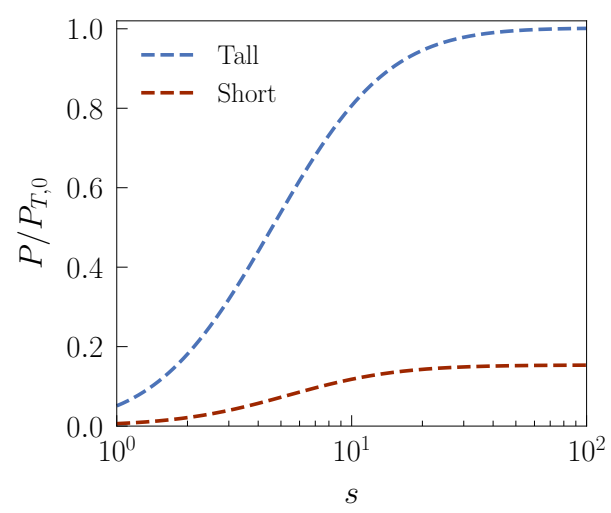

(b)

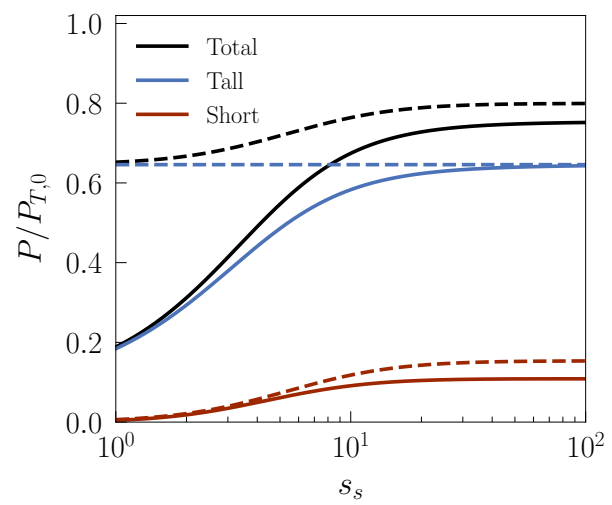

Figure 2.5: Average turbine power output in a wind farm normalized by a free-standing tall turbine for (a) two vertically aligned wind farms as a function of the dimensionless turbine spacing and (b) separate vertically aligned wind farms (dashed) and both combined making a vertically staggered wind farm (solid) while varying the short turbine spacing.

be observed using this top-down approach as opposed to the commonly used bottom-up models. More specifically, despite the fact that the tall turbine spacing remains constant, the relative power of the tall turbines in the vertically staggered case is influenced by the presence and spacing of the short turbines. The relative power of the tall turbines approaches the value of the tall vertically aligned case when the short turbine spacing is large and their impact is negligible. Using this model, we are able to gain insight into which short turbine spacing allows tall turbines in a vertically staggered wind farm to perform approximately as they would without additional short turbines. We also observe that the relative power of the short turbines within the vertically staggered wind farm also increases as the spacing increases but remains less than the vertically aligned case due to interactions with the fixed tall turbines. Nonetheless, both scenarios are similar at small spacing, when the short turbines are very dense, suggesting that the influence of the tall turbines is less in this case. When comparing the total average contribution of both short and tall turbines, the vertically aligned case is more productive. That being said, two separate vertically aligned cases are expected to perform better than combining the two wind farms into the vertically staggered scenario because of the increase in the total turbine density, having twice the amount of turbines interacting and extracting flow momentum. This result emphasizes that the costs of implementing these configurations should be considered for a more useful comparison when quantifying the benefits of vertically staggered wind farms. 


\subsection{Optimizing inter-turbine spacing}

We observed that a vertically staggered wind farm can be more productive than a vertically aligned wind farm. Here, we address whether this benefit outweighs the additional expense by considering simplified costs which would accompany placing short turbines into a layout of tall turbines. Specifically, we quantify the normalized power per unit cost and normalized profit per unit cost.

\subsubsection{Normalized power per unit cost}

We normalize the power by simplified costs in order to determine the optimal spacing at which short turbines should be placed within a wind farm. Specifically, we consider the land cost, the linear costs (cables, etc.), and the turbine costs. Following the approach of Stevens et al. [58], we define the normalized power per unit cost as

$$
P^{*}=\frac{P}{P_{0}} \frac{1}{1+\beta s+\theta s^{2}},
$$

where $s=\sqrt{s_{x} s_{y}}$ is the dimensionless turbine spacing and the dimensionless cost coefficients are

$$
\theta=\frac{\text { Cost }_{\text {land }}}{\text { Cost }_{\text {turbine }} / D^{2}}, \quad \beta=\frac{\text { Cost }_{\text {linear }}}{\text { Cost }_{\text {turbine }} / D} .
$$

$P^{*}$ is calculated while varying the dimensionless turbine spacing $\left(s_{T}\right.$ or $s_{S}$ ) for $\theta$ values deemed reasonable by references $[57,58]$. The spacing at which the normalized power per unit cost is the highest is selected as the optimal spacing $s_{\text {opt }}$. The optimal spacing for each $\theta$ is shown in Figure 2.6 (a) for tall or short turbines belonging to independent vertically aligned wind farms (Figure 2.3 a) and (b)). We observe that when $\theta$ is high, i.e. land cost is high or turbine cost is low, turbines should be placed closer together. In contrast, when $\theta$ is low, turbines should be placed farther apart. Furthermore, we observed that our results agree with Meyers and Meneveau [57] when the linear costs $\beta$ are excluded. In addition, we observe that although the difference is small, at each $\theta$, a wind farm with taller turbines should ideally have turbines placed closer together than in the case with short turbines.

When considering a vertically staggered wind farm, the normalized power per unit cost will depend on the relative power contribution of both the tall and short turbines in the unit. When adding and adjusting the spacing of the short turbines, we 
(a)

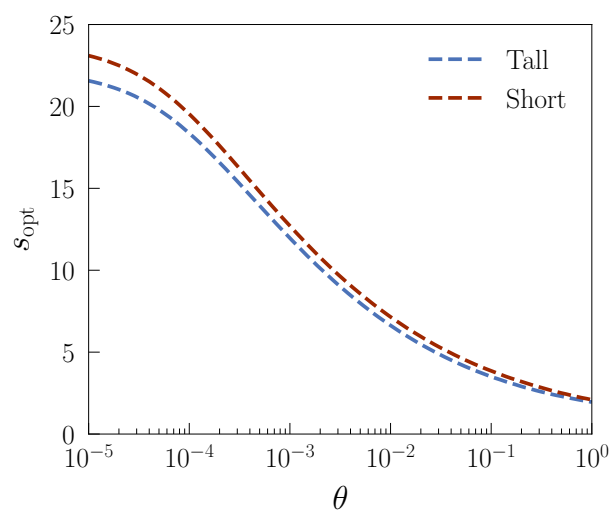

(b)

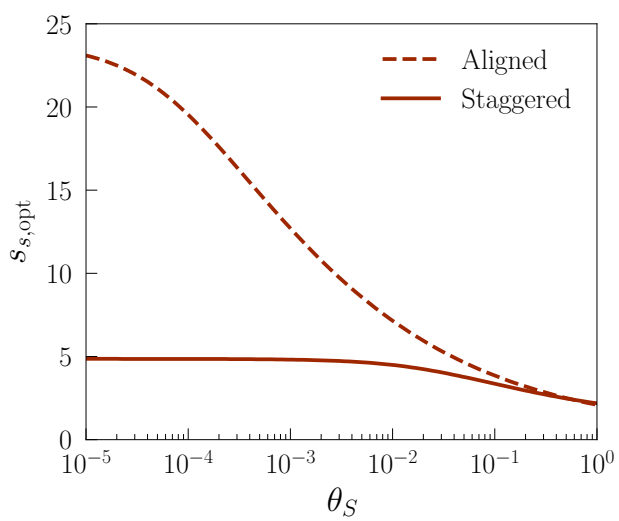

Figure 2.6: The optimal spacing of turbines based on the maximum normalized power per unit cost for (a) two vertically aligned wind farms (Figure $2.3(\mathrm{a})$ and (b)), and (b) for a vertically aligned farm containing only short turbines compared to the same short turbines within a vertically staggered wind farm (Figure $2.3 \mathrm{~b}$ ) and (c)).

must consider a unit such that $P_{\text {unit }}=N_{T} P_{T}+P_{S}$ where the tall turbine density is $N_{T}=S_{S}^{2} / S_{T}^{2}$, in order to compare to the vertically aligned case with only short turbines. The cost of both the short and tall turbines must be included and we assume Cost $_{\text {turbine, } T}=\left(D_{T}^{2} / D_{S}^{2}\right)$ Cost $_{\text {turbine }, S}$ for simplicity. Therefore, the modified expression for normalized power per unit cost for a vertically staggered wind farm is:

$$
P^{*}=\frac{P_{\text {unit }}}{P_{0, \text { unit }}} \frac{1}{1+\left(\frac{s_{S}^{2}}{s_{T}^{2}}\right)+\beta s_{S}+\theta s_{S}^{2}} \text {, where } \theta_{S}=\frac{\text { Cost }_{\text {land }}}{\text { Cost }_{\text {turbine }, \mathrm{S}} / D_{S}^{2}} .
$$

In Figure 2.6(b), the vertically staggered and vertically aligned wind farms are compared to determine the optimal separation of short turbines while the tall turbines remain fixed $\left(s_{T}^{2}=7.85 \times 5.24\right)$. At high $\theta$, the optimal spacing is low and similar for both the vertically aligned and staggered cases as the cost of land is high and turbines are ideally placed closer together. However, it is evident that as $\theta$ decreases, the optimal spacing of the short turbines becomes limited when tall turbines are present. Moreover, at low $\theta$ when the cost of turbines is high, if the short turbine spacing increases, the fraction of tall turbines included in the unit increases and the cost of these turbines outweighs the benefit of further reducing the wake interaction. Though simplified, this model provides useful insight into the basic trends and which short turbine spacing is most suitable in a vertically staggered farm to offset the costs. 


\subsubsection{Normalized profit per unit cost}

Ultimately, power translates into revenue which must be higher than the costs in order for a wind farm to be profitable. We estimate the profitability of vertically staggered wind farms by considering the revenue, the cost of the wind turbines, linear costs (cables etc.), and land costs. The profit of a vertically staggered wind farm when considering the area occupied by a tall turbine as a unit is defined as follows:

$$
\text { Profit }=\text { Revenue }_{\text {total }}-\text { Cost }_{\text {total }},
$$

Revenue $_{\text {total }}=C_{p} \times\left(P_{T}+N_{S} P_{S}\right) \times$ years $\times$ electricity rate,

$$
\operatorname{Cost}_{\text {total }}=\text { Cost }_{\text {turbine }, T}+N_{S} \text { Cost }_{\text {turbine }, S}+\operatorname{Cost}_{\text {linear }} S_{T}+\operatorname{Cost}_{\text {land }} S_{T}^{2} \text {, }
$$

where $C_{p}$ is the capacity factor and $N_{S}=S_{T}^{2} / S_{S}^{2}$ is the number of short turbines per tall turbine. We normalize the profit by the cost of a tall turbine, and again assume that Cost $_{\text {turbine }, T}=\left(D_{T}^{2} / D_{S}^{2}\right)$ Cost $_{\text {turbine }, S}$, arriving at the normalized profit per unit cost:

$$
\text { Profit } *=\frac{\text { Profit }}{\text { Cost }_{\text {turbine }, T}}=\left(\frac{P_{T}+N_{S} P_{S}}{P_{T, 0}}\right) \zeta-\left(1+\left(\frac{s_{T}}{s_{S}}\right)^{2}+\beta s_{T}+\theta s_{T}^{2}\right),
$$

where

$$
\theta_{T}=\frac{\text { Cost }_{\text {land }}}{\text { Cost }_{\text {turbine }, T} / D_{T}^{2}}, \quad \zeta=\frac{C_{p} \times \text { years } \times \text { electricity rate } \times P_{T, 0}}{\text { Cost }_{\text {turbine }, T}} .
$$

We plot this expression per unit area (Profit* $/ s_{T}^{2}$ ) for a vertically staggered wind farm case in Figure 2.7 (a) as a function of $\theta$ where the tall turbine spacing remains constant and the short turbine spacing is varied. Figure 2.7 (a) also displays the first and second terms in Equation 2.37per unit area which reflect the normalized revenue and the normalized cost, respectively. We observe that when $s_{S}$ is small, the unit is not profitable because of the increased wake interaction and increased turbine costs. However, the unit is profitable at larger $s_{S}$. In fact, there is a local maximum indicating the optimal spacing of short turbines. As $s_{S}$ increases past this point, the profitability decreases approaching an asymptote which represents the case when only tall turbines are present. Therefore, in Figure 2.7 (b), we normalize by the corresponding vertically aligned control case with tall turbine which remain fixed.

Figure 2.7 (b) shows the normalized profit per unit cost divided by the result of the control case without added short turbines for a range of $\zeta$. We observe that the normalized profit per unit cost is greater than one for a range of $s_{S}$, suggesting that within 
(a)

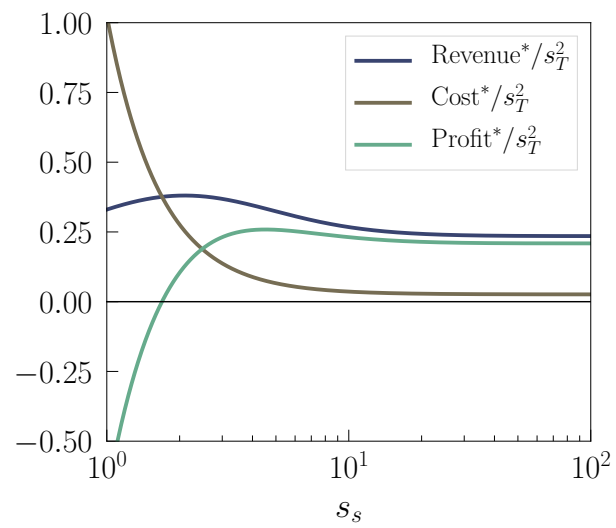

(b)

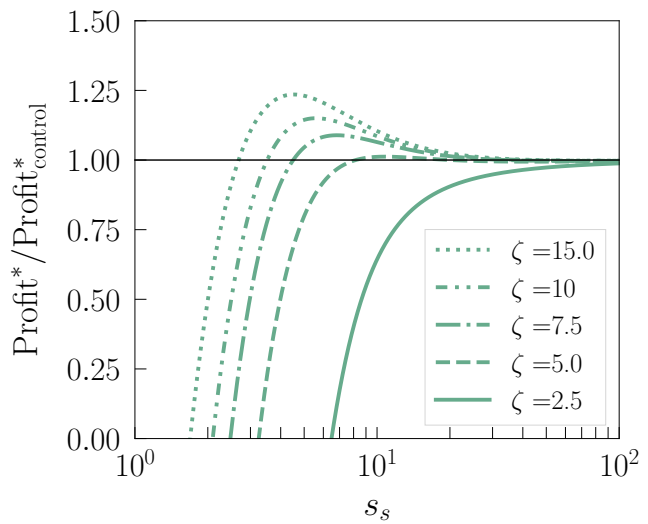

Figure 2.7: Normalized profit per unit cost of a vertically staggered farm as a function of the short turbine spacing where the tall turbine spacing remains constant and $\theta_{T}=10^{-3}$, (a) $\zeta=15.0$ and (b) a parameter scan for $\zeta$ where the result is normalized by the vertically aligned control case without short turbines.

this range, this vertically staggered wind farm is more profitable than the control case. When $s_{S}$ is very large, the result approaches one and the unit performs equivalent to the control case as alluded in Figure 2.7 (a). We observe that when $\zeta$ is larger, a wider range of $s_{S}$ values make a profitable wind farm, and the spacing at which the farm is most profitable also shifts. For instance, when $\zeta=7.5$, the short turbines are ideally placed at $s_{s, \mathrm{opt}}=6.85$, meanwhile when $\zeta=15.0$, the optimal spacing is $s_{s, \mathrm{opt}}=4.57$. Essentially, when the turbines generate more revenue, there are optimally more short turbines in the unit. We note that previous economic cost analysis [58, 60] suggested that for most wind farms $\zeta<2$. However, this value can vary per wind farm site and over time. For example, given the developments of wind energy and the increasing demand for clean energy, a more favorable economic situation for wind farms may be possible in the future [61]. Therefore, the development of simple analytical models that can assess large ranges of the parameter space is useful. Overall, we have demonstrated that this simple model gives some insight into the potential profitability of vertically staggered wind farms. 


\subsection{Conclusions}

In this study, we provide an overview of a top-down analytical model which we have applied to multi-scale wind farm optimization. We investigated the power production of various vertically staggered wind farm configurations. Additionally, we performed a simplified cost analysis showing that introducing short turbines into a tall turbine array may not be more profitable than the tall turbines alone. To achieve this, we built upon a well-established analytical model for infinite vertically staggered wind farms by considering short horizontal axis turbines with a vertical gap between the tall and short turbines' rotor-swept areas, as well as incorporating the upper limitation of an IBL. This top-down model is a useful tool because of its simplicity and low cost, and in contrast to bottom-up models, this approach accounts for large-scale interactions.

To exhibit the capabilities of this simple model, we explored the effect of the short turbine's diameter, hub-height, and spacing as well as the roughness scale, on the productivity of vertically staggered wind farms. First, we compared the relative power output of the tall and short turbines to a control case without short turbines. When varying the diameter and hub-height of the short turbines, we observed that though smaller turbines may benefit from reduced wake losses, their overall contribution becomes less substantial. Therefore, minimizing the size of the short turbines is not necessarily favorable because the advantage of being farther from the ground and having a larger rotor-swept area outweighs the hindrance of increased wake exposure. We also observed that the assigned surface roughness scale influences the result and vertical staggering appears to be more impactful in offshore conditions. Next, we investigated the influence of the turbine density, finding that when the number of short turbines per tall turbine increases, the wind farm's productivity increases as there are more turbines generating power. However, this gain is not linear because of the increased wake interactions.

We then focused on the turbine spacing and evaluated the relative power compared to a tall free-standing turbine. Specifically, we varied the turbine spacing for tall and short turbines as separate arrays (vertically aligned) and then combined into one wind farm (vertically staggered). We observed that the relative power output of both tall and short turbines in individual vertically aligned wind farms was higher than when they are combined into a vertically staggered wind farm due to the negative influence of increased wake interactions. Although two separate wind farms with a lower turbine density are expected to produce more power, this arrangement would be costlier. Hence, these results highlight the importance of also considering the costs when determining the potential benefit of a vertically staggered layout.

There are many approaches to quantifying the benefit of vertically staggered wind farms. We studied the normalized power per unit cost paralleled with Stevens et al. 58 
as well as the normalized profit per unit cost. When determining at which short turbine spacing the normalized power per unit cost is optimized, we observed that the optimal spacing is limited by the presence of the tall turbines, compared to the vertically aligned wind farm with only short turbines. Moreover, despite the vertical gap between their rotor-swept areas, the short turbines are influenced by the tall turbines extracting kinetic energy. We performed a case study in which we considered the revenue of the vertically staggered wind farm, and subtracted the costs to analyze the normalized profit per unit area. We found that the vertically staggered wind farm was only profitable for a certain range of short turbine spacing and that a local maximum existed, indicating that the vertically staggered farm would be more profitable than the vertically aligned case with only tall turbines. However, adding the short turbines was only profitable when the revenue was scaled higher than currently available values. Therefore, this insight suggests our particular multi-scale wind farm configuration is not profitable which may be related to the infinite-nature of the wind farm. Nonetheless, future wind farm development could consider vertically staggered arrangements depending on the relevant cost coefficients.

We have shown that simple analytical models are useful for investigating multiple vertically staggered wind farm configurations and elucidating basic trends with ease. We have given a glimpse of the potential uses of this model but there are many possible enhancements that can be made. This model could be applied further as to retroactively fit current wind farms with even larger turbines as turbine technology advances in this direction. In the future, it would also be useful to compare these results to higher-fidelity simulations and to explore denser turbine layouts. The model itself could be further enhanced by incorporating thermal stratification or downstream development [56]. Specifically, we would adapt the model to consider large finite-size vertically staggered turbine arrays instead of infinite wind farms by introducing the IBL depth; as proposed by [31, 32]. Nonetheless, although these results are not directly reflective of real wind farms, this model is useful considering the balance between accuracy and computational cost, and can give an indication of what may be worth investigating with higher-fidelity models or experiments. 


\section{Chapter 3: LARGE EDDY SIMULATIONS OF VERTICALLY STAGGERED WIND FARMS}

Vertically staggering wind turbines, such that some are taller than others, can mitigate wake losses and take advantage of undisturbed flow. We contribute to the understanding of the fluid physics involved in multi-scale wind farms by using high-fidelity large eddy simulations to explore the benefit of adding smaller turbines to a fixed array of tall turbines. Primarily, we investigate the influence of the relative placement and density of the additional short turbines in both onshore and offshore atmospheric conditions. The performance of the vertically staggered layouts is compared to a reference case with only tall turbines in order to isolate the impact of the added short turbines. Our findings suggest that although short turbines may hinder the productivity of tall turbines due to increased wake interactions, their power contribution outweighs this impediment, even when four short turbines are sub-optimally placed directly downstream of each tall turbine. We also observe that a combination of horizontal and vertical staggering is more productive than vertical staggering alone. Specifically, short turbines contributed more to the cumulative power output when placed between the tall turbines as opposed to directly downstream, emphasizing the importance of their relative placement. Finally, we find that vertical staggering is more beneficial in offshore conditions because the tall turbine wakes recover slower than in onshore conditions, yet the contribution of the short turbines is similar in both cases. 


\subsection{Introduction}

Wind farms are being installed at an increasing capacity and occupying more area, stressing the importance of optimizing wind farm layouts [4]. When a turbine extracts momentum from the wind, it generates a wake downstream. This wake is characterized by a velocity deficit and high turbulence intensity, which ultimately decreases the kinetic energy available for subsequent turbines and increases their structural load. Accordingly, wind turbines produce more power when their wake interactions are minimized. Wakerelated power losses can be avoided by sufficiently separating the turbines so that the wake recovers through entrainment before reaching downstream turbines. However, increasing turbine spacing also increases operational costs. Staggering the turbines is an alternative method to reduce wake interactions by simply offsetting the rotor-swept areas of succeeding turbines. In this way, staggering reduces wake exposure and takes advantage of undisturbed flow without requiring more land. Horizontal staggering is a common and relatively well-explored approach $33,34,36$. However, vertical staggering, such that some turbines are shorter than others (i.e. Figure 3.2 d)), is less established. In general, adding short turbines to existing wind farms may increase overall productivity, but more research is required to support this as a viable option for real wind farms.

Simulations are a useful tool for evaluating wind farm design prior to construction by studying the physics which ultimately determine the wind farm's productivity. Chatterjee et al. [38] used large eddy simulations, coupled with the actuator line method, to investigate multi-scale wind farms by adding short turbines with two different hub-heights to an array of tall turbines. Supporting the findings of Xie et al. [37], Chatterjee et al. [38] claimed that large coherent structures generated by the tall turbines can make short turbines more productive than if the short turbines were alone. Though insightful, these simulations were limited by a small domain size and periodic boundary conditions which lack realistic inflow. Zhang et al. [39.42] also investigated vertically staggered wind farms using large eddy simulations. In contrast to Chatterjee et al. [38], their simulations applied the actuator disk method in a larger domain and provided more realistic inflow conditions using the concurrent precursor method 62]. They asserted that the maximum benefit of vertical staggering is at the entrance region. However, Zhang et al. 39. 42] considered a uniform turbine diameter without a vertical gap between the rotor-swept areas of tall and short turbines. Therefore, their vertically staggered turbines are subjected to significant wake overlap. Chatterjee et al. [38] suggested such overlap would be detrimental because intermediate-height turbines would not benefit from large coherent structures. Wu et al. 43] evaluated staggered layouts with less overlap, also using large eddy simulations coupled with the actuator disk method. However, similar to Chatterjee et al. [38, they also use streamwise periodic boundary conditions. Therefore, in our study, we use large 
eddy simulations with the actuator disk model and concurrent precursor method while maintaining a vertical gap between short and tall turbines.

We investigate the benefit of vertically staggered wind farms while focusing on a few key parameters: the roughness scale, the short turbine spacing, and the relative position of the short turbines. We explore a limited number of cases because large eddy simulations are more computationally-expensive and time-consuming than the analytical model used in Chapter 2 Nonetheless, indications from the analytical model are quite useful for selecting which variables may be of most interest for higher-fidelity simulations. For instance, in Chapter 2 we observed that advantage of adding short turbines depends on factors such as the short turbine density (number of short turbines per unit area) and the surface roughness. However, the analytical model could not distinguish the relative position of the short turbines. Therefore, in this study, we investigate the scenario in which short horizontal axis turbines are added to a fixed array of tall turbines while systematically varying the relative position and number of the short turbines. We perform these simulations in both onshore and offshore atmospheric conditions by adjusting the corresponding roughness scale to $z_{0}=0.1 \mathrm{~m}$ and $z_{0}=0.001 \mathrm{~m}$, respectively [22]. Specific simulation details are provided in Section 3.2 The results and comparison of these cases are discussed in Section 3.3 followed by a summary in Section 3.4

\subsection{Numerical framework}

\subsubsection{Large eddy simulations}

We perform pressure-driven large eddy simulations for a neutrally-stratified atmospheric boundary layer $(\mathrm{ABL})$ providing detailed spatial and temporal information for large-scale wind farms. The updated large eddy simulation code is based on the version developed by Albertson and Parlange [63 64]. This code solves the isothermal, incompressible NavierStokes equations above a certain grid scale $(\tilde{\Delta})$ while the effects of smaller structures are modeled $[65$. The filtered values are denoted with a tilde $(\sim)$. This method is suitable for large-scale atmospheric studies because large structures are the most frequent, while small structures are the most intensive to resolve [19]. Pairing the conservation of momentum with the conservation of mass (the incompressible continuity equation), the governing equations in index notation are as follows:

$$
\begin{gathered}
\partial_{t} \tilde{u}_{i}+\partial_{j}\left(\tilde{u}_{i} \tilde{u}_{j}\right)=-\partial_{i} \tilde{p}^{*}-\partial_{j} \tau_{i j}-\delta_{i 1} \partial_{1} p_{\infty} / \rho+f_{i}, \\
\partial_{i} \tilde{u}_{i}=0,
\end{gathered}
$$

where $\tilde{u}_{i}$ is the filtered velocity field and $i=1,2,3$ are the axial direction components corresponding to $x, y$, and $z$, respectively. The turbine forcing $f_{i}$ is modeled using the 
actuator disk method which will be described in more detail below. The molecular viscosity has been neglected due to the high Reynolds number $\left(\sim O\left(10^{8}\right)\right)$ [25.26. Also, real ABL flow is driven by geostrophic forcing, but for simplicity, we neglect system rotation (Coriolis forces) and the simulations are driven by an imposed pressure gradient in the $x$ direction $\partial_{1} p_{\infty} / \rho$, where $p_{\infty}$ is the averaged pressure and $\rho$ is the density of air [18]. We note that the assumption of a pressure-driven boundary layer is equivalent to assuming a given strength for the transverse geostrophic wind. This approach has been shown to give meaningful results for wind farms as the flow in the inner region (up to $15-20 \%$ of the boundary layer height) is not influenced by external effects such as Coriolis forces [18 23 66. The remaining terms are the filtered modified pressure $\tilde{p}^{*}=\tilde{p} / \rho+\tau_{k k} / 3-p_{\infty} / \rho$ and the sub-grid scale stress term $\tau_{i j}=\widetilde{u_{i} u_{j}}-\tilde{u}_{i} \tilde{u}_{j}$. We model the traceless part of this term using the scale-dependent Lagrangian dynamic model such that

$$
\tau_{i j}-\delta_{i j} \tau_{k k} / 3=-2 \nu_{T} \tilde{S}_{i j}=-2\left(C_{\text {smag }} \Delta\right)^{2}|\tilde{S}| \tilde{S}_{i j}
$$

where $\nu_{T}$ is the turbulent eddy viscosity and $\tilde{S}_{i j}=\frac{1}{2}\left(\partial_{j} \tilde{u}_{i}+\partial_{i} \tilde{u}_{j}\right)$ is the resolved strain tensor. The eddy viscosity is modeled as $\nu_{T}=\left(C_{\text {smag }} \Delta\right)^{2}|\tilde{S}|$ where $|\tilde{S}|=\left(2 \tilde{S}_{i j} \tilde{S}_{i j}\right)^{1 / 2}$ and $C_{\text {smag }}$ is the Smagorinksy coefficient, which is calculated dynamically using the method outlined in reference 67.

The governing equations are solved as follows. The time integration is performed using a second-order accurate Adams-Bashforth scheme. In the vertical direction, a secondorder accurate finite difference discretization is applied with the following boundary conditions. At the top, a stress-free, zero vertical velocity boundary condition is enforced. At the bottom boundary, we impose a free-slip condition where the wall stress is related to the velocity of the first grid point using the classic logarithmic law for turbulent wall-bounded flow 67,68 . The simulations are horizontally homogeneous and a pseudospectral discretization is applied with doubly-periodic boundary conditions. However, the periodicity in the streamwise direction, which would create an infinitely long wind farm, is negated using the concurrent precursor method to achieve realistic atmospheric inflow conditions [62].

The concurrent precursor method involves simulating two domains simultaneously as illustrated in Figure 3.1 A turbulent ABL domain is generated, which is used as inflow for a second domain with a wind farm. Each time step, velocity sampled from the ABL domain, is introduced to the wind farm domain via a fringe region which comprises $9 \%$ of the streamwise domain length. We use this fringe region, in combination with a shifting technique [69] and long time-averaging, to limit the effect of high-velocity wind speed streaks. For further details on this method, we refer the reader to reference [62]. An example of an ABL domain without turbines is plotted in Figures 3.2 (a), (b) and 


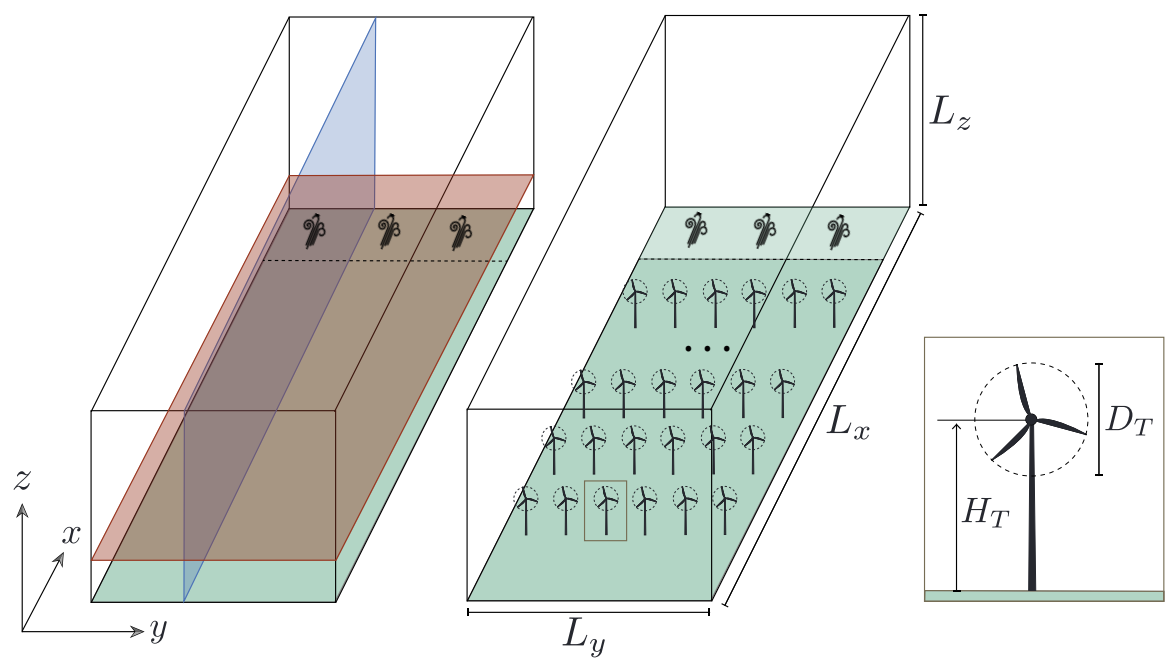

Figure 3.1: Schematic of the two domains generated when using the concurrent precursor method: an ABL without turbines (left) and an ABL with turbines (right). Data from the fringe region (dashed line) is transferred every time step from the inflow domain to the wind farm domain. The red and blue planes illustrate horizontal and vertical cross-sections for plotting, i.e. in Figure 3.2 The tall turbine diameter $D_{T}$ and hub-height $H_{T}$ are also shown.

(c), which are vertical and horizontal cross-sections of the instantaneous streamwise velocity normalized by the friction velocity $u_{*}$. The turbulent nature of the inflow ABL is quite evident and the velocity increases with distance from the surface. In fact, if horizontally-averaged, we would arrive at the idealized velocity profile described in the classic log-law wind profile for turbulent wall-bounded flow. Figures 3.2(c), (d), and (e) show cross-sections of the instantaneous streamwise velocity for a corresponding wind farm domain with both short and tall turbines. Distinctly, the flow behind each turbine is slower and more turbulent than the inflow, which is classified as a wake. The wakes generated by the tall turbines are stronger than the short turbine wakes.

As mentioned, the turbines are modeled using the actuator disk method [71]. Although more detailed models exist, such as the actuator line method [72], they are computationally-intensive because they require a high resolution and are therefore not feasible for large-scale wind farm studies. Furthermore, successive turbines are often placed within the far-wake region $(3-10 D)$, which is less dependent on the detailed turbine features and more on global parameters such as the thrust [23, 73]. The actuator disk method is sufficient for modeling the far-wake region and is therefore applicable for wake development and vertical staggering studies \begin{tabular}{r|l|l|}
18 & 23 & 66
\end{tabular} . The standard actuator disk 
(a) $y=1.3 \mathrm{~km}$

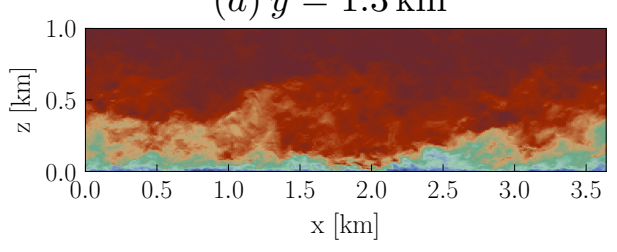

(b) $z=70 \mathrm{~m}$

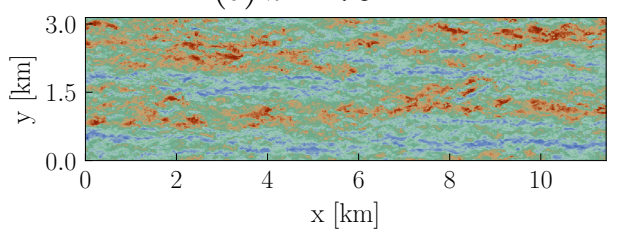

(c) $z=150 \mathrm{~m}$

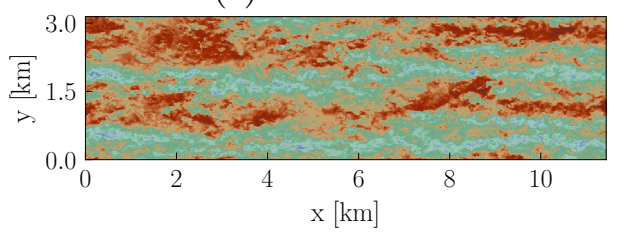

(d) $y=1.3 \mathrm{~km}$

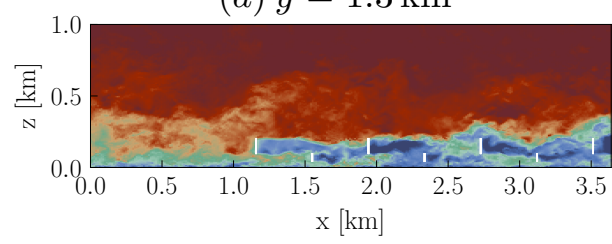

(e) $z=70 \mathrm{~m}$

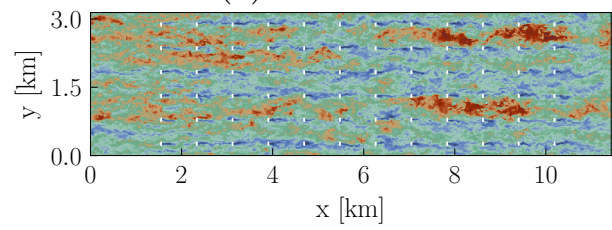

$(f) z=150 \mathrm{~m}$

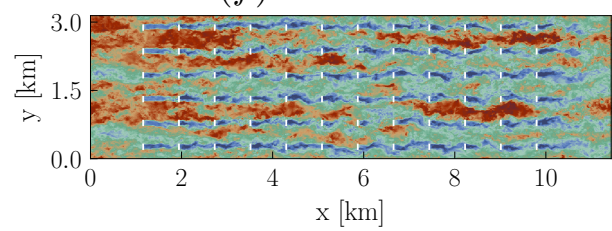

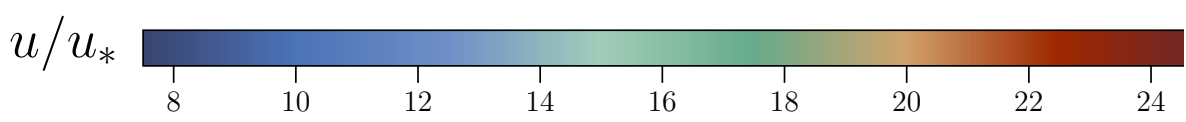

Figure 3.2: The instantaneous streamwise velocity of the inflow domain (a, b, c) and the corresponding wind farm domain $(\mathrm{d}, \mathrm{e}, \mathrm{f})$ where $z_{0}=0.1 \mathrm{~m}$. The wind farm layout is the TS-D1 (refer to Table 3.1 and Figure 3.5, with an equal number of tall and short turbines. Figures (a, c) are the vertical $x-z$ cross-sections along the centerline of the third column of turbines. Figures $(\mathrm{b}, \mathrm{d})$ are horizontal $x-y$ cross-sections at the short turbine hub-height $z=H_{S}$, and (e,f) at the tall turbine hub-height $z=H_{T}$. 


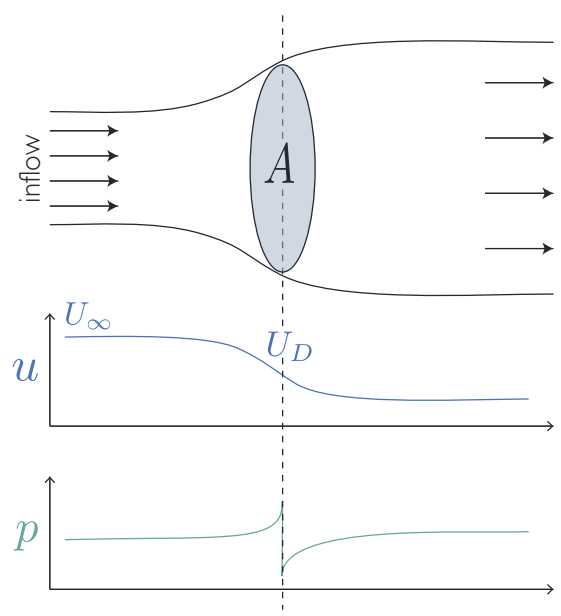

Figure 3.3: A depiction of actuator disk theory 40, 70]: Streamtube with a disk of area $A$ which exerts a force on the flow are shown alongside the corresponding evolution of streamwise velocity $u$, and the change in pressure $p$, in front of and behind the disk.

theory defines the thrust force exerted by the disks as

$$
F_{T}=-\frac{1}{2} \rho C_{T} U_{\infty}^{2} A,
$$

where $C_{T}=4 a(1-a)$ is the thrust coefficient, $a$ is the axial induction factor, $A=$ $\pi D^{2} / 4$ is the rotor-swept area, and $U_{\infty}$ is the undisturbed upstream velocity $18,71.74$ 75]. The disk, alongside the corresponding relative changes in streamwise velocity and pressure, is sketched in Figure 3.3 .

The upstream velocity is not readily available when simulating turbulent flows and is impossible to define for subsequent turbine rows. Therefore, we substitute the local disk-area-averaged velocity $U_{D}$, using actuator disk theory such that

$$
C_{T}^{\prime}=\frac{C_{T}}{(1-a)^{2}}, \text { where } a=\frac{U_{\infty}-U_{D}}{U_{\infty}} .
$$

The turbine power output is then determined using $P=-F_{T} U_{D}$, expressed as

$$
P(t)=\frac{1}{2} \rho C_{T}^{\prime} U_{D}(t)^{3} \frac{\pi}{4} D^{2}
$$

More detail is available in references [74,76]. Although the actuator disk model is not an exact representation of the turbines, it is widely used and provides valuable insight when the thrust coefficient is lower than the Betz limit $\left(C_{T}=8 / 9 ; a=1 / 3 ; C_{T}^{\prime}=2\right)$ $18,23,66$. For this particular set of simulations, we apply the filtered actuator disk method 


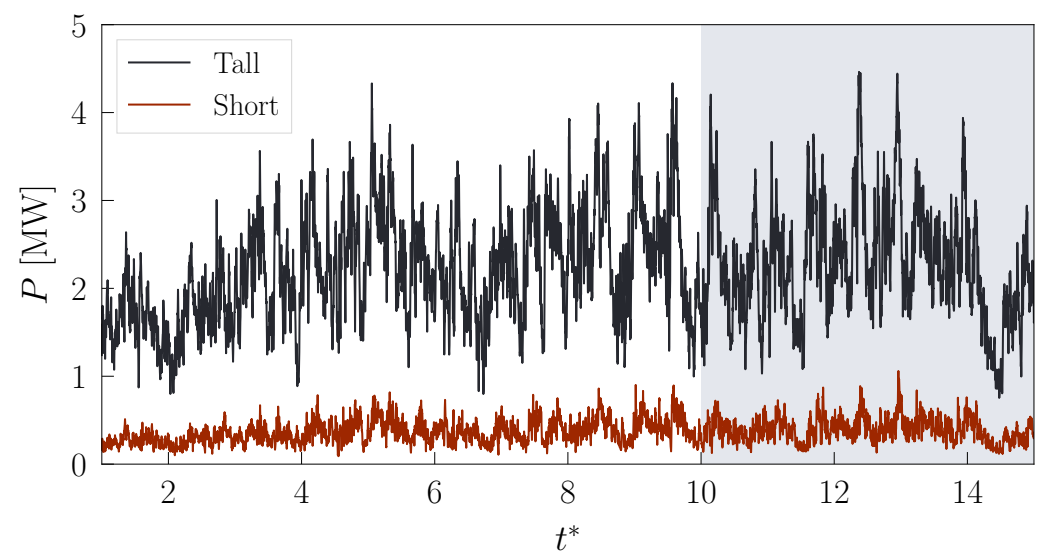

Figure 3.4: Power output as a function of time for two turbines within the TS-D1 wind farm (refer to Table 3.1) where $z_{0}=0.1 \mathrm{~m}$. Time-averaging of the simulation is performed during $t^{*}=t u_{*} / H=10-15$, which is indicated by the shaded region.

developed by Shapiro et al. [77]. The disks are oriented perpendicular to the inflow for both simplicity regarding statistics and to study the worst-case scenario regarding wake interactions. An example of the power output as a function of time is presented in Figure 3.4 for a tall and short turbine in a vertically staggered wind farm. Although the power clearly fluctuates over time, the average value is dependent on the turbine parameters, which is visible when comparing the short and tall turbines. In the following sections, we address trends using time-averaged statistics. We begin time-averaging once the simulation develops and the flow is fully turbulent in both domains, as shown in Figure 3.2 In this particular study, we average between time units $t^{*}=\left(t u_{*} / H\right)=10-15$ and time-averaged values are denoted using a bar $\left({ }^{-}\right)$. Horizontally-averaged values exclude the fringe region and are denoted using triangle brackets $(\langle\rangle)$. 


\subsubsection{Simulation specifications}

We simulate five different wind farm layouts with two different surface roughness scales: $z_{0}=0.1 \mathrm{~m}$ and $z_{0}=0.001 \mathrm{~m}$, corresponding to onshore and offshore conditions respectively [22]. These cases are listed in Table 3.1 and depicted in Figure 3.5 We investigate the potential benefit of adding shorter turbines to a fixed wind farm and therefore, the reference case is the fixed wind farm with only tall turbines. Based on indications from Chapter 2 we explore how the density of shorter turbines affects the potential advantage of vertically staggered wind farms. Thus, for the vertically staggered layouts, we adjust the ratio of tall to short turbines as either $N_{T}: N_{S}=1: 1$ or $1: 4$. Furthermore, the analytical model could not account for the relative positioning of the short turbines. Therefore, we briefly explore this factor by placing the short turbines either directly behind the tall turbines (TS-D), or in between the tall turbines (TS-L). The TS-L layout can benefit from both horizontal and vertical staggering and being in the first row, while the TS-D configurations can only benefit from vertical staggering.

The turbine and domain parameters are summarized as follows. The disk-based thrust coefficient is $C_{T}^{\prime}=4 / 3$ as this work originated from previous research which also used this value [78. The dimensions of the short and tall turbines are selected based on the Chapter 2 findings: we aim to minimize wake interactions without placing the short turbines too close to the ground. Therefore, the short turbine hub-height and diameter $\left(H_{S}=70 \mathrm{~m}, D_{S}=50 \mathrm{~m}\right)$ are selected to be as large as possible without overlapping vertically with the tall turbines $\left(H_{T}=150 \mathrm{~m}, D_{T}=100 \mathrm{~m}\right)$. The constant dimensionless spanwise and streamwise spacing of the tall turbines is $s_{T, y}=5.24$ and $s_{T, x}=7.85$, respectively, in accordance with typical values for existing wind farms. There are 12 tall turbine rows $\left(N_{T, x}=12\right)$, and six turbines in each of those rows $\left(N_{T, y}=6\right)$, totaling $N_{T}=72$ tall turbines. The dimensionless domain lengths are $L_{x}, L_{y}, L_{z}=(4 \pi, \pi, 1.0) / H$, where $H=1000 \mathrm{~m}$ is the assigned ABL height for neutral conditions [18]. The horizontal dimensions are sufficiently large, relative to the height of the domain, that the wind farm is considered infinite in the spanwise direction due to the periodic boundary conditions [57]. The domain is discretized uniformly by the number of grid points $N_{x}, N_{y}, N_{z}=1024,256,240$, such that the resolution is $\Delta_{x}, \Delta_{y}, \Delta_{z}=12.3 \mathrm{~m}, 12.3 \mathrm{~m}, 4 \mathrm{~m}$. Therefore, the rotor area of the small turbines is discretized with at least 38 grid points and the large turbines have approximately 160 grid points. For more detail regarding sufficient resolution we refer the reader to references [34, 79, 81].

We explore vertical staggering in both onshore and offshore conditions. Adjusting

the roughness scale affects the velocity profile as described in Chapter 1 Section 1.2. We established that the velocity normalized by the corresponding friction velocity is lower in onshore conditions than offshore conditions at the same height. Furthermore, the 


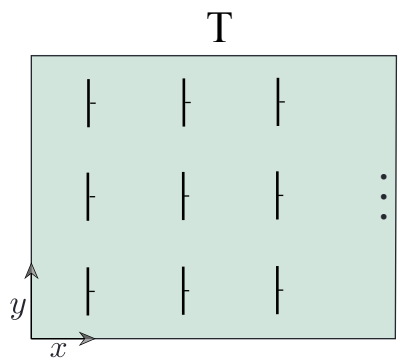

TS-D1

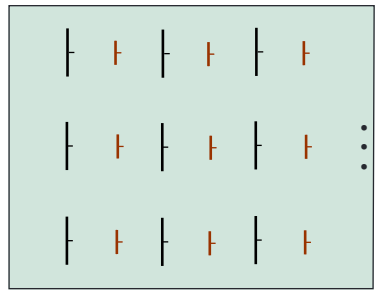

TS-D4

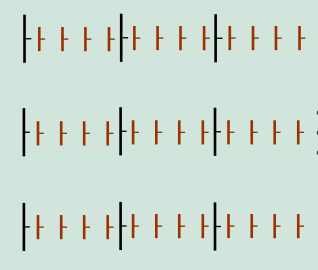

TS-L1

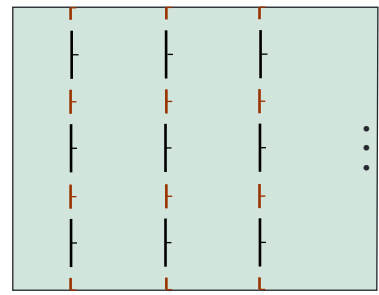

TS-L4

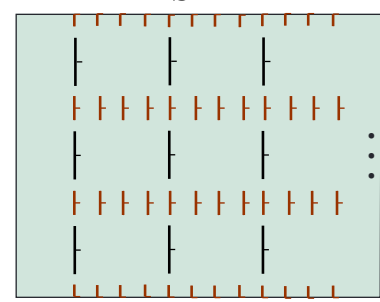

Figure 3.5: A top-view schematic of the relative positioning of short turbines (red) with respect to the fixed tall turbines (black) for the five different turbine layouts considered.

\begin{tabular}{llc}
\hline Layout reference & Short turbine placement & $N_{T}: N_{S}$ \\
\hline T & - & $1: 0$ \\
TS-D1 & Downstream & $1: 1$ \\
TS-L1 & Lateral & $1: 1$ \\
TS-D4 & Downstream & $1: 4$ \\
TS-L4 & Lateral & $1: 4$ \\
\hline
\end{tabular}

Table 3.1: List of vertically staggered wind farm simulations executed for both $z_{0} / H=10^{-4}$ and $z_{0} / H=10^{-6}$, corresponding to onshore and offshore conditions, respectively, totaling ten cases. The short turbine placement is the relative position with respect to the reference tall turbines as illustrated in Figure 3.5 The ratio of the number of tall turbines to the number of short turbines is $N_{T}: N_{S}$. 


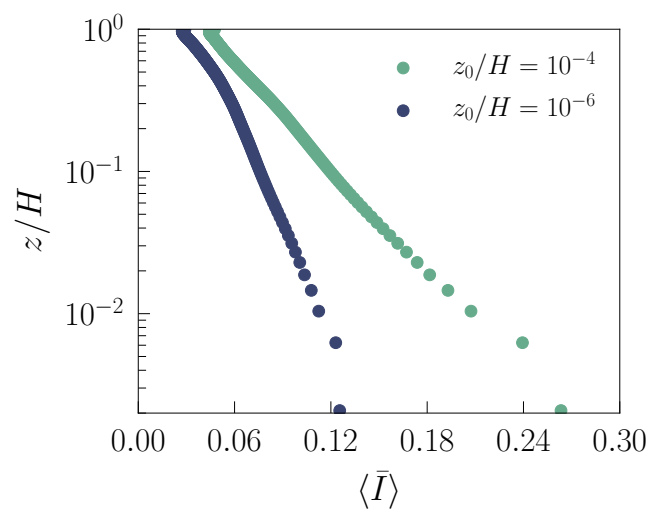

Figure 3.6: Time- and horizontally-averaged streamwise turbulence intensity as a function of normalized height $z / H$ for both onshore and offshore conditions.

assigned roughness scale influences the amount of turbulence in the atmosphere. The streamwise turbulence intensity is defined as

$$
I=\frac{\sqrt{\left\langle\left(u^{\prime}\right)^{2}\right\rangle}}{\langle u\rangle} \text {, where } u^{\prime}=u-\bar{u}
$$

The turbulence intensity of an ABL without turbines is compared for both onshore and offshore conditions in Figure 3.6 as a function of the normalized height. The turbulence intensity decreases farther away from the rough surface and onshore conditions are more turbulent than offshore conditions. This trend is expected $[66,82,-84]$ and because wake recovery is driven by turbulent vertical mixing, the surface roughness will ultimately affect the power production of downstream turbines, which we discuss in Section 3.3

\subsection{Results and discussion}

We begin by exploring how vertically staggered wind farms influence the ABL by analyzing the time-averaged streamwise velocity. Then, we evaluate the potential benefit of vertically staggered wind farms by examining the power output. We focus on how the relative performance is dependent on the short turbine spacing and relative placement, as well as considering the impact of onshore and offshore ABL conditions. 


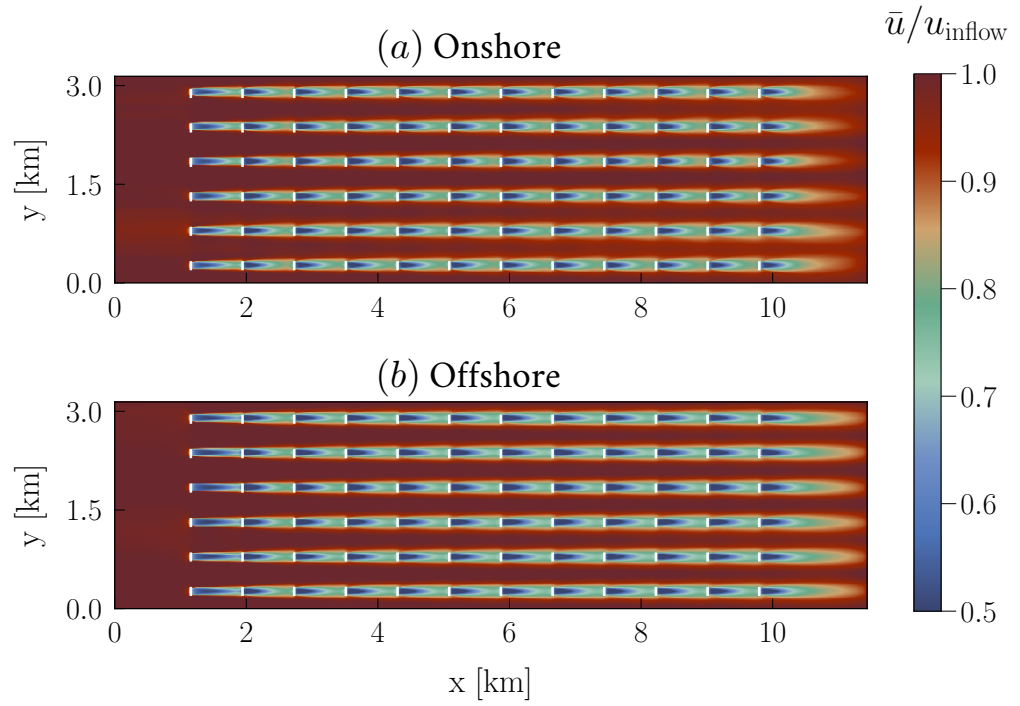

Figure 3.7: The time-averaged streamwise velocity at hub-height $\left(z=H_{T}\right)$ for the reference case $(\mathrm{T})$ normalized by the horizontally-averaged value in the inflow domain at hub-height for onshore $\left(z_{0} / H=10^{-4}\right)$ and offshore $\left(z_{0} / H=10^{-6}\right)$ ABL conditions.

\subsubsection{Time-averaged streamwise velocity}

Wind turbines extract kinetic energy from the flow, creating a turbulent wake that develops downstream, as shown in Figure 3.2. Streamwise velocity is the dominant component determining the power output. Therefore, in Figure 3.7 we show the horizontal crosssection of the time-averaged streamwise velocity at hub-height for the reference case without turbines. Figures 3.7 a) and (b) show the same wind farm, but in onshore and offshore ABL conditions corresponding to the roughness scales $z_{0} / H=10^{-4}$ and $z_{0} / H=10^{-6}$, respectively. In order to compare the different simulations, the velocity data is normalized by $u_{\text {inflow }}$, which is the horizontally-averaged streamwise velocity at hub-height in the inflow domain. First, the turbulent structures observed in Figure 3.2 are no longer visible in Figure 3.7 due to the time-averaging. Nonetheless, the turbine wakes are evident, characterized by downstream velocity deficits that recover as they progress downstream. Second, comparing Figures 3.7 (a) and (b), we observe that the wakes are stronger in offshore conditions. The low velocity flow is replenished by entrainment of higher velocity flow from above and if the inflow is more turbulent, then there is a higher turbulent entrainment flux 83. Therefore, wakes recover more quickly in onshore conditions due to the stronger vertical mixing. 

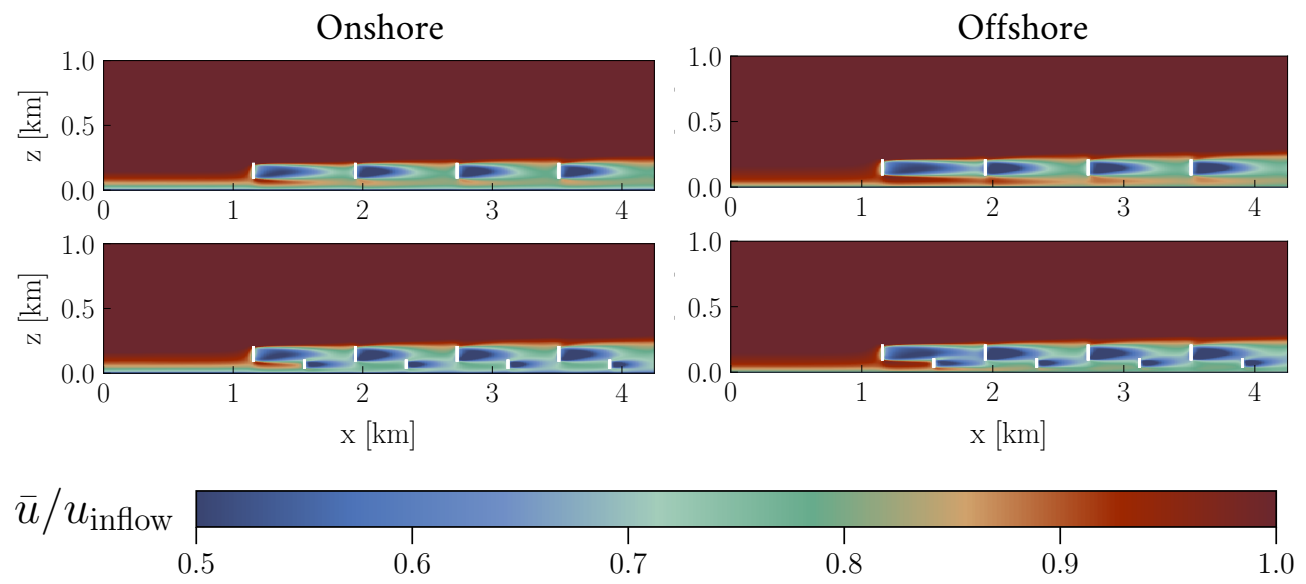

Figure 3.8: The time-averaged streamwise velocity at $y=1.3 \mathrm{~km}$ (the axial centerline of the third column of turbines) normalized by the time- and horizontally-averaged value in the inflow domain at $z=H_{T}$, for the T and TS-D1 layouts in (a) onshore, and (b) offshore ABL conditions.

Vertical staggering can mitigate wake losses by placing turbines underneath the strongest part of the velocity deficit and taking advantage of relatively undisturbed flow below the taller turbines. The vertical range of the wake is visible in Figure 3.8 which shows a vertical cross-section of the normalized streamwise velocity along the centerline of the third column of turbines. The streamwise velocity is shown for the reference wind farm (T) and a vertically staggered wind farm (TS-D1), for both onshore and offshore conditions. We observe that, despite avoiding the bulk of the tall turbines' wake, the short turbines are placed in a low velocity region which is apparent by comparing the reference case. When turbines are placed closer to the ground, the normalized velocity is lower due to the roughness of the surface and corresponding negative shear effect. However, the first row of short turbines receives a relatively high velocity flow compared to the subsequent rows, especially in the offshore case with lower surface roughness. Therefore, the productivity of short turbines is expected to be higher at the front of the wind farm. Downstream, the momentum extraction of tall and short turbines clearly influences the kinetic energy available for consecutive turbines when the turbine density has increased.

Figure 3.9 compares the normalized time- and horizontally-averaged streamwise velocity profile for each simulation listed in Table 3.1 Figure 3.9 (a) and (b) display the results for onshore and offshore conditions, respectively. In both figures, there are clear deviations from the reference case without short turbines for all vertically staggered layouts, indicating that more momentum is being extracted. For instance, when the short turbines are added, the velocity decreases within the short turbine rotor-swept range. 

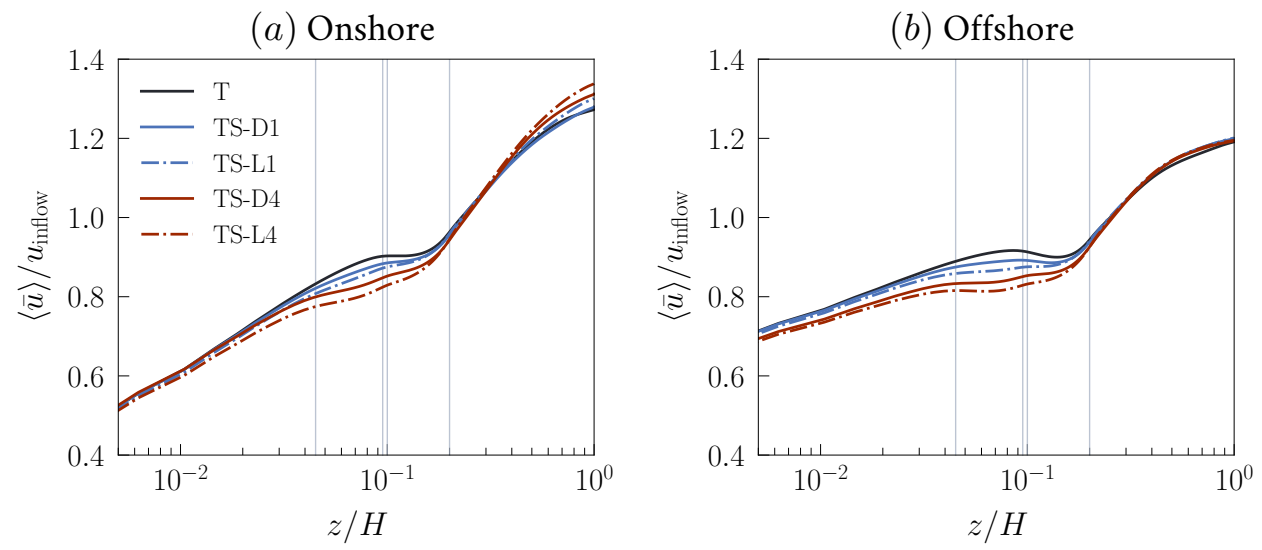

Figure 3.9: Time- and horizontally-averaged streamwise velocity profile for the wind farm domain normalized by the value in the inflow domain at hub-height for (a) onshore, and (b) offshore ABL conditions. The vertical lines denote the extent of the rotor-swept areas of short $\left(H_{S}-D_{S} / 2 \leqslant z \leqslant H_{S}+D_{S} / 2\right)$ and tall $\left(H_{T}-D_{T} / 2 \leqslant z \leqslant H_{T}+D_{T} / 2\right)$ turbines.

Also, the velocity is lower within the tall turbine rotor-swept range because the short turbines extract additional momentum. Generally, the velocity deviates even more from the reference case when increasing the short turbine density from $N_{T}: N_{S}=1: 1$ to $1: 4$, because more momentum is extracted. In fact, when the number of short turbines is increased, the velocity is lower than the reference case even near the top edge of the tall turbine range $\left(z=H_{T}+D_{T} / 2\right)$.

In addition, the relative position of the short turbines impacts the velocity profile in Figure 3.9 We observe that when the turbine density is equal, the TS-L layouts have a lower velocity than the TS-D layouts within the rotor-swept areas. The velocity decreases because more momentum is being extracted in the TS-L cases. Short turbines that are placed between the tall turbine columns receive higher velocity flow than short turbines that are placed directly in-line with the tall turbines, despite them being shorter than the bulk of tall turbine wake. Concurrently, the tall turbines are less influenced by short turbines. When four short turbines are placed directly behind each other, they are subjected to each other's wakes, but nonetheless, more kinetic energy is available to them if they are placed between the tall turbine columns. Therefore, combining the benefits of horizontal and vertical staggering can bring about more power production for both tall and short turbines by further reducing wake losses.

Overall, these deviations from the reference case without short turbines are larger in offshore conditions. Essentially, the negative effect of shear on turbines placed closer to the ground is stronger when the surface roughness is higher in onshore conditions. The 
deviations in momentum extraction from the reference case are clearly dependent on the short turbine spacing and relative position as well as the atmospheric conditions, which will ultimately be reflected in the power production.

\subsubsection{Power production of vertically staggered wind farms}

We evaluate the effectiveness of adding short turbines to a fixed array of tall turbines by comparing the time-averaged power output of a vertically staggered wind farm (TS) to the reference case without short turbines $(\mathrm{T})$. Since the turbine rows are spanwise-infinite, we consider the average power of each row. We begin by normalizing the performance of turbines in each row by the first row at the entrance of the farm which is not subjected to wake effects.

First, we address how the productivity is impacted by the ABL conditions. Figure 3.10 a) shows the relative power for the reference case in both onshore and offshore $\mathrm{ABL}$ conditions corresponding to $z_{0} / H=10^{-4}$ and $z_{0} / H=10^{-6}$, respectively. In both cases, we observe that the subsequent rows do not perform as well as the first row due to momentum extraction and wakes created by the upstream turbines. In fact, downstream turbines can produce $30 \%-50 \%$ less power than the first row (which is not exposed to wakes). Further downstream, the consecutive rows have a similar relative power due to the balance between the kinetic energy extraction and entrainment of higher velocity flow from above [18 62 85]. Comparing onshore and offshore conditions, we observe that the decrease in relative power production of the downstream turbines is more pronounced in offshore conditions than in onshore conditions. Essentially, wake recovers more quickly in onshore conditions because the turbulence intensity is higher, facilitating entrainment. Furthermore, there is less variation and a higher relative power in onshore conditions suggesting that the balance between wake production and destruction is achieved more quickly in this case.

The ABL conditions similarly influence the relative power output of the vertically staggered layouts, as shown in Figures $3.10 \mathrm{~b}$-e). The pattern of vertical staggering is obvious as the power output of most short turbine rows is less than $20 \%$ of the first row of tall turbines. The dramatic difference is in large part due to their small size and proximity to the rough surface where the wind velocity is low because of the shear (refer to Figure 1.4). The relative power production of the short turbine rows is even less for the dense cases where $N_{T}: N_{S}=1: 4$ because there is less kinetic energy available for each turbine to extract. Regarding the $\mathrm{ABL}$ conditions, the relative power of the downstream tall turbine rows is higher in onshore conditions for each wind farm layout due to increased vertical mixing and wake recovery. In contrast to the tall turbines, the relative performance of most short turbine rows is similar for both onshore and offshore 


$$
\begin{aligned}
& z_{0} / H=10^{-4} \\
& -z_{0} / H=10^{-6}
\end{aligned}
$$

(b) TS-D1

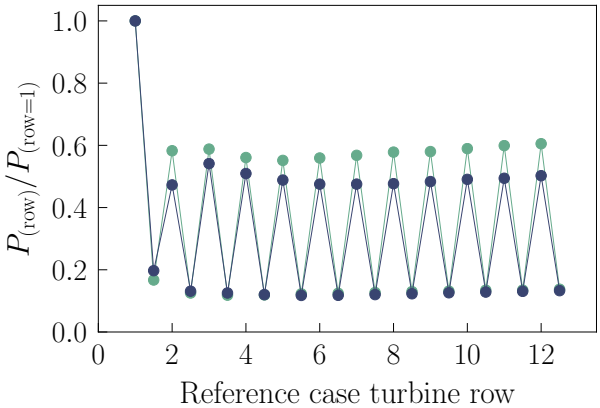

(d) TS-D4

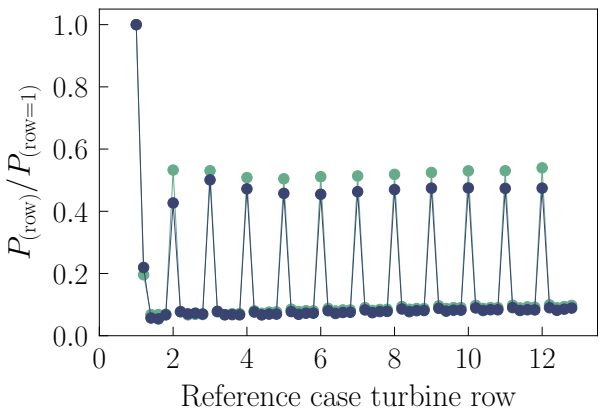

(a) $\mathrm{T}$

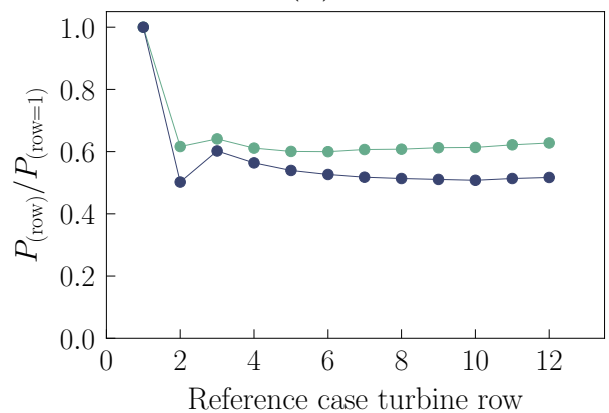

(c) TS-L1

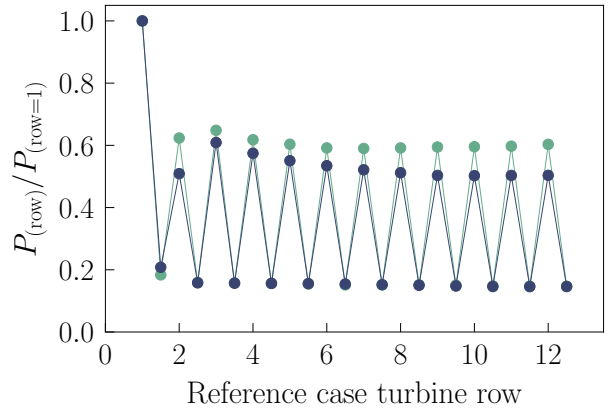

(e) TS-L4

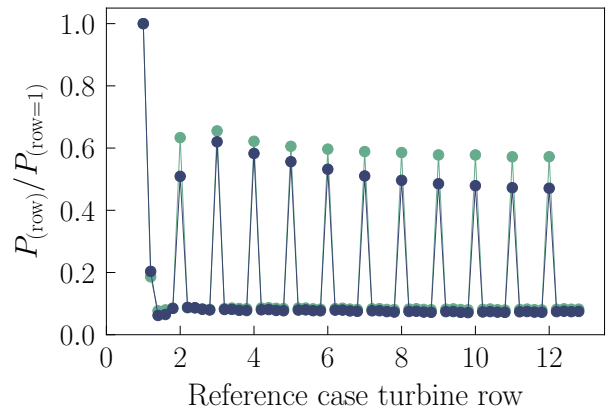

Figure 3.10: Average power output of wind turbine rows normalized by the first row for each of the simulations listed in Table 3.1 in onshore and offshore ABL conditions corresponding to $z_{0} / H=10^{-4}$ and $z_{0} / H=10^{-6}$, respectively. 
(a) $N_{T}: N_{S}=1: 1$

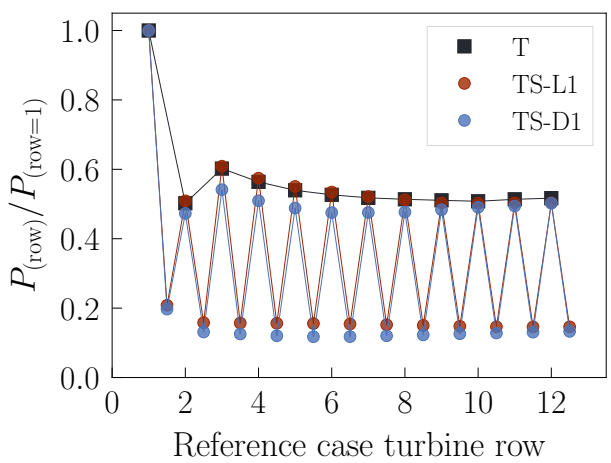

(b) $N_{T}: N_{S}=1: 4$

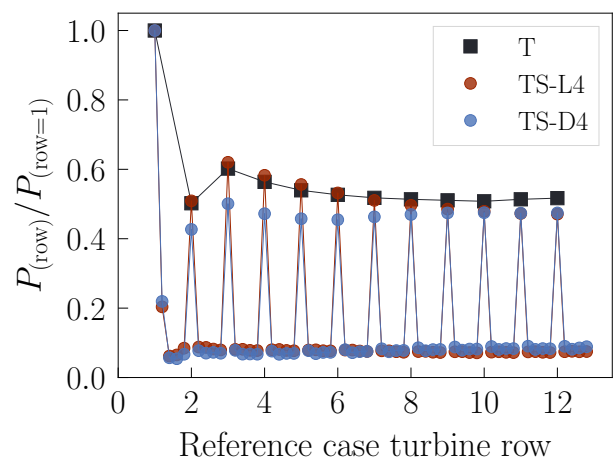

Figure 3.11: Average power output of wind turbine rows normalized by the first row for the wind farms listed in Table 3.1 where $z_{0} / H=10^{-6}$.

conditions. The short turbines reside in a low velocity region and are more sensitive to local wake development. The first row of short turbines is an exception, performing better than downstream short turbine rows, and is influenced by the ABL conditions. This behavior agrees with Figure 3.8. which shows that the first row is exposed to the least amount of wake and therefore relatively undisturbed, higher velocity inflow. Thus, we expect that the entrance of the wind farm will be the most impacted by vertical staggering. Overall, the surface roughness influences the relative power production of vertically staggered wind farms through changes in turbulent mixing. The larger difference in relative power production of the tall turbines in offshore conditions suggests they could benefit more from the short turbines which are relatively unaffected.

Next, we focus on how the relative placement of short turbines (i.e. downstream or in between the tall turbines) affects the relative performance of the wind farm layouts. Vertically staggered wind farms with the same turbine density and roughness scale are plotted together in Figure 3.11 alongside the reference case without short turbines. In Figure 3.11 a), we observe that placing short turbines directly downstream of tall turbines (TS-D1) negatively impacts the relative power of tall turbine rows near the entrance of the wind farm, despite the fact that the rotor-swept areas do not overlap. Essentially, the momentum extraction of the short turbines influences what is available for the tall turbines. In the same figure, we observe that the tall turbines are not notably impacted by the short turbines when they are placed in between the tall turbines (TS-L1). The short turbines also perform relatively better in the TS-L1 case than when they are placed directly downstream because their exposure to the tall turbine wakes is further negated through horizontal staggering. In this case, kinetic energy extraction is less localized. 
Nonetheless, the difference between the two layouts diminishes farther downstream. We observe similar trends when $z_{0} / H=10^{-4}$.

In Figure 3.11(b), the short turbine density increases to $N_{T}: N_{S}=1: 4$. Overall, we observe that adding more short turbines can decrease the relative power production of both tall and short turbine rows because of increased wake interactions. For both TS-D4 and TS-L4, we observe that most short turbine rows generate less than $10 \%$ of the power of the first tall turbine row. Furthermore, the short turbines perform similarly in both cases because they are placed directly downstream and are vertically aligned with respect to each other. The relative power production of the tall turbine rows decreases when more short turbines are placed directly downstream (TS-D4), but this deviation from the reference case subsides farther downstream. The tall turbine performance is similar to the reference case when the added short turbines are placed in between the tall turbine columns (TS-L4). Essentially, when the short turbine density increases, additional horizontal staggering can reduce wake losses for the first few rows, but the relative performance of the tall turbines is not sensitive to short turbine placement in the developed region of the wind farm. Therefore, considering this behavior and the relatively high contribution of the first short turbine row, vertical staggering is most impactful at the entrance of the wind farm, supporting the findings of Zhang et al. 39, 42. Considering a potential decrease in the relative performance of tall turbines, and low relative power production of short turbines, one might assume that such a multi-scale wind farm is not advantageous. However, though the short turbines may not produce as much as tall stand-alone turbines, there are more turbines in total contributing to the overall wind farm productivity in the vertically staggered layouts. So, it begs the question: what is their cumulative contribution?

To evaluate the benefit of vertical staggering, we quantify the cumulative power contribution that short turbines make to the potentially hindered tall turbines. We observed that surface roughness, turbine density, and relative position of the short turbines, influence momentum extraction and wake interactions. The gain in cumulative power compared to the reference case without short turbines is

$$
\Delta P=\left(P_{\text {cum }, \mathrm{TS}}-P_{\text {cum }, T}\right) / P_{\text {cum }, T} .
$$

The result for each configuration is listed in Table 3.2 . Across the board, adding short turbines to the reference case creates a positive gain in cumulative power, asserting that vertical staggering is more productive than if the tall turbines are alone. As anticipated, the relative benefit is dependent on each of the variables of interest. For all turbine layouts, the offshore wind farms benefit more from adding short turbines, supporting the findings of Vasel-Be-Hagh et al. [52]. When turbines are placed close to the ground, they are exposed to less wake, but experience power losses due to negative shear effects related to the surface roughness. Concerning the relative position of the turbines, we observe that 


\begin{tabular}{lcc}
\hline Layout & $\Delta P\left(z_{0} / H=10^{-4}\right)$ & $\Delta P\left(z_{0} / H=10^{-6}\right)$ \\
\hline TS-D1 & $14.1 \%$ & $16.5 \%$ \\
TS-L1 & $21.9 \%$ & $28.1 \%$ \\
\hline TS-D4 & $37.8 \%$ & $44.2 \%$ \\
TS-L4 & $48.3 \%$ & $54.9 \%$ \\
\hline
\end{tabular}

Table 3.2: Cumulative power gain of vertically staggered configurations (TS) compared to the reference case without short turbines $(\mathrm{T})$.

placing short turbines between the tall turbine columns (TS-L) is more productive than placing them directly behind the tall turbines (TS-D), reaping the wake mitigation benefits of both vertical and horizontal staggering. For instance, at the entrance of the wind farm, where vertical staggering provides the most benefit, the laterally placed turbines are not subjected to any streamwise wake. Wake interactions increase when the short turbine density increases, yet we observe more gain when $N_{T}: N_{S}=1: 4$ compared to $N_{T}: N_{S}=1: 1$. Even though these turbines do not perform relatively well at high density, the fact that there are so many turbines generating power adds up to an overall benefit, especially when considering the plateauing of relative power production that develops downstream.

We compare the gain observed in Table 3.2 to the results of the analytical model presented in Chapter 2 Figure 2.4 The analytically derived gain for $H_{S}=70 \mathrm{~m}, D_{S}=$ $50 \mathrm{~m}, z_{0} / H=10^{-4}$, is $\Delta P \sim 12 \%$ when $N_{T}: N_{S}=1: 1$ and $\Delta P \sim 36 \%$ when $N_{T}: N_{S}=1: 4$. These values agree well with the (TS-D) layouts here using the higherfidelity large eddy simulations. However, in general, the gain of adding small turbines is not linear, i.e. $4 \times \Delta P(\mathrm{TS}-\mathrm{X} 1) \neq \Delta P(\mathrm{TS}-\mathrm{X} 4)$, highlighting the importance of considering the expenses which accompany such layouts. For instance, does a gain of $54.9 \%$ offset the accompanying costs of adding 4 times as many turbines, even if they are smaller? Future studies should evaluate whether the costs of adding the short turbines is offset by the gain. 


\subsection{Conclusions}

The purpose of this study was to investigate the benefit of vertically staggering wind turbines using large eddy simulations. Adding shorter turbines to a fixed array of tall turbines increases wind farm productivity by avoiding wake losses and taking advantage of relatively undisturbed flow $38,39,42]$. Overall, we observed that the short turbines yield a positive net contribution to the total power output, offsetting any negative impact on the tall turbines. Complementary to what we found using the analytical top-down model in Chapter 2 we investigated the influence of the ABL conditions and the density of short turbines while also now distinguishing the impact of their relative position.

We demonstrated that adding short turbines to an array of tall turbines is advantageous. The first row of short turbines receives a relatively undisturbed flow; however, the subsequent short turbines receive low-velocity inflow even if they are avoiding the bulk of tall turbine wakes. The wind is inherently low velocity near the surface and these short turbines also suffer from interactions with each other, especially at high density. By studying the relative power of the turbine rows, we found that the tall turbines are less impacted by mutual wake interactions in onshore conditions due to increased vertical mixing. However, the contribution of short turbines is less sensitive to the $\mathrm{ABL}$ conditions and more dependent on the internal boundary layer region created by the tall turbines. Moreover, the cumulative power gain showed that the vertical staggering is more beneficial in offshore conditions and agreed well with the analytical model results.

We determined that the relative placement of the short turbines influences the benefit of vertical staggering, which could not be captured using the analytical model in Chapter 2 Specifically, when short turbines were placed laterally in between the tall turbine columns, they benefited from horizontal and vertical staggering and the first short turbine row operates in free-stream conditions. The relative placement of the short turbines was less influential downstream, even when the short turbine density was high. Nonetheless, we observed that the cumulative power gain is substantial even when four short turbines are placed directly downstream of the tall turbines and the effect of vertical staggering is isolated (i.e. horizontally aligned). The power contribution of the short turbines, even if performing poorly due to the low velocity and the short turbine wake interactions, offset any negative impact on the tall turbine's relative performance.

As the demand for renewable energy grows and space for wind farm development becomes limited, future wind farm planning should consider adding smaller turbines to existing wind farms. Our results show that vertically staggered wind farms can be more productive and that the gain is dependent on the surface roughness, as well as the density and relative position of the short turbines. The established relationships between wind farm design and productivity encourages investigation into the expenses that accompany 
these different layouts and whether they are economically viable. Future work should consider these costs, coupled with existing wind farm layouts, to elucidate the applicability of vertical staggering. We also recommend adapting the large eddy simulation code to model vertical axis wind turbines $[86-89]$, and potentially applying different thrust coefficients for tall and short turbines. Finally, exploring the feasibility of retro-fitting existing tall turbine arrays with even taller turbines may be interesting as modern turbines become increasingly larger. 


\section{Part II}

\section{Flow blockage in large-scale wind turbine arrays}





\section{Chapter 4: BLOCKAGE EFFECTS IN LARGE WIND TURBINE ARRAYS IN A NEUTRAL BOUNDARY LAYER}

Understanding how blockage affects the entrance of large-scale wind farms is essential because the first row produces the most power and is often used as a reference for the subsequent rows. We use large eddy simulations to investigate the blockage effects caused by close spanwise and streamwise turbines by comparing a stand-alone turbine, an infinite row of turbines, and a wind farm with eight rows. We find that flow blockage in dense turbine arrays is highly dependent on the dimensionless turbine spacing. Spanwise neighboring turbines appear to benefit from deflected flow, while close downstream turbines hinder the upstream turbines. In this case, the wind farm acts as an obstruction and flow is inclined to pass over the wind farm, reducing the productivity of the first row. In agreement with the uniform inflow wind tunnel experiments by Segalini and Dahlberg [90], we find that the effect of downstream turbines follows a universal trend as a function of the average inter-turbine spacing when the performance of the first wind farm row is normalized with the corresponding solitary row case. However, we also demonstrate that the particular wind farm layout strongly affects the cumulative blockage as the results do not follow a universal trend when normalized with a stand-alone turbine. Furthermore, we show that blockage is affected by the turbine hub-height but varies less than $1 \%$ when comparing onshore and offshore conditions.

Adapted from submission to: Jessica M. I. Strickland and Richard J. A. M. Stevens, Investigating wind farm blockage in a neutral boundary layer using large eddy simulations, European Journal of Mechanics / B Fluids. 


\subsection{Introduction}

Wind farms are installed at an increasing rate and turbines are ideally placed as close together as possible to limit costs. However, adequate turbine spacing is necessary because reduced power production is a consequence of cramped wind farms. Optimizing the turbine spacing can be done prior to construction by understanding the flow physics in and around wind farms. While negative downstream effects, such as turbine wake interactions are well-established [23, 66], other phenomena, such as blockage effects, have only recently received more attention from the wind energy community. These blockage effects are not yet included in many widely-used wind farm models, although first attempts in this direction are made 91. A better understanding of wind farm blockage is crucial as these effects lead to systematic deviation in power production estimates.

Flow blockage is a phenomenon in which incoming wind deviates, avoiding an obstruction. In this way, turbines influence the incoming flow, potentially altering the kinetic energy available for neighboring turbines. An individual turbine has an inherent preceding velocity deficit compared to the undisturbed flow, known as induction. Blockage effects can be observed in the front of a wind farm, where the turbines are typically assumed to receive undisturbed flow and perform the same as isolated turbines. However, limited but pertinent studies have indicated that blockage may affect wind farm performance in certain conditions, suggesting that neighboring turbines may benefit or hinder each other, depending on their relative positions.

The induction zone preceding a single turbine is characterized by a velocity deficit. The velocity $U$ along the symmetry axis is well described by the following analytical expression obtained from actuator disk theory,

$$
\frac{U}{U_{\infty}}=1-a\left[1+\frac{x}{\left(x^{2}+R^{2}\right)^{1 / 2}}\right],
$$

where $U_{\infty}$ is the undisturbed upstream velocity, $x$ is the distance upstream, $R=D / 2$ is the rotor radius, and $a$ is the axial induction factor [40, 41]. Medici et al. [92] supported this classical result using a rotating wind turbine model and wind tunnel experiments, asserting that the incoming flow can be affected up to three diameters upstream for a single turbine. The above relationship has also been confirmed in field measurements [93-95], while Forsting et al. [96] validated Reynolds-averaged Navier-Stokes (RANS) results for the induction region against lidar measurements. Later, Forsting et al. 97] compared a simple induction zone model to large eddy simulations using the actuator line method and found good agreement between these methods.

Limited blockage research has extended beyond a stand-alone turbine. Nonetheless, placing turbines closer together in either the spanwise or streamwise direction 
may influence the incoming airflow differently. This work defines spanwise blockage as the observed power production increase caused by closely-spaced spanwise turbines. McTavish et al. [98] performed wind tunnel experiments, determining that three closelyspaced turbines within a row can benefit each other via spanwise blockage, observing an increased average turbine power output of up to $10 \%$. Although susceptible to wind tunnel blockage, such experiments are very insightful. Forsting et al. [99] studied spanwise blockage using RANS and a vortex model. Their results also showed that having close spanwise-neighboring turbines increases the average power output compared to a stand-alone turbine.

Some studies have addressed the potential blockage effects caused by downstream turbines. Ebenhoch et al. [100] developed a linearized model for wind farm flows, comparing to experimental data, and observed effects of blockage up to $30 D$ in front of a wind farm. Bleeg et al. [101] performed RANS and observed that wind farm scale blockage causes a wind-speed slowdown of $1.9 \%$ at $7-10 D$ upstream and found reasonable agreement between their model results and field observations. Recently, Branlard et al. [102 103 used engineering-type models to account for the wind farm blockage effect. For a $5 \times 5$ wind farm with $5 D$ inter turbine spacing, they showed that the flow velocity $2.5 D$ upstream of a wind farm can be reduced by $2 \%$. Nygaard et al. [91] introduced a wind farm blockage model based on the accumulation of single-turbine induction effects, which captures the blockage trends observed in field measurement data but underestimates the magnitude of the effect. Bleeg [104] suggested employing RANS models in combination with a graph neural network approach to model turbine interaction losses and blockage because traditional wake models tend to ignore blockage effects. Segalini et al. 90, 105] studied wind farm scale blockage in wind tunnel experiments and showed that the front row of a wind farm is negatively impacted by the presence of downstream turbines such that

$$
\frac{U}{U_{\text {ref }}}=1-0.097\left(\frac{S_{x} S_{y}}{D^{2}}\right)^{-0.9}\left[1-\exp \left(0.88-0.88 N_{T, x}\right)\right],
$$

where $D$ is the turbine diameter, $S_{x}$ and $S_{y}$ are the dimensional streamwise and spanwise spacing, respectively, and $N_{T, x}$ is the number of turbine rows. The reference velocity $U_{\text {ref }}$ is the velocity of the first row when all successive rows are placed $32 D$ downstream. Sebastiani et al. [106] evaluated blockage effects in the Nysted wind farm using field measurements and found the effect to be about $2 \%$, which is consistent with the above formula. They also assessed blockage using WindSim, OpenFOAM, and ORFEUS and, depending on the model and wind direction, the estimated effect is between $2-4 \%$.

More experiments and simulations are required to get a better fundamental understanding of wind farm blockage. Various of the cited studies considered idealized test-cases or a limiting number of conditions. For example, the work by Segalini and 
Dahlberg [90 105] considers uniform inflow and therefore it is unclear to what degree their findings are applicable to wind farms in an atmospheric boundary layer (ABL). Limited field data is available to validate these results; therefore, we use high-fidelity large eddy simulations as a tool which balances accuracy and computational cost to assess blockage effects in various large-scale wind farm configurations in a neutrally-stratified, highly-turbulent ABL. We use large eddy simulations to ensure that the effects due to turbulent mixing, which are not fully represented in RANS, are accounted for. We study the effects of spanwise and streamwise blockage systematically by considering a standalone turbine, an infinite row of turbines, and a wind farm with eight rows. Generally, we use spanwise-infinite rows, but the role of the spanwise extent is also briefly explored. Primarily, we investigate the dependence of the blockage effect on the turbine density (number of turbines per unit area), the turbulence intensity at hub-height, and the turbine hub-height. We determine the effect of the streamwise and spanwise turbine spacing on the performance of turbines in the first row of a wind farm in order to assess the impact of the wind turbine layout on wind farm blockage. Specifically, we consider the effects of flow blockage on the relative power output of turbines and the induction region. The remainder of this chapter is organized as follows. The numerical framework and specific layout and characteristics of the simulations are outlined in Section 4.2 . The results for these cases are then presented in Section 4.3 and further discussion and conclusions are summarized in Section 4.4

\subsection{Numerical framework}

\subsubsection{Large eddy simulations}

High-fidelity large eddy simulations provide detailed time-dependent data suitable for analyzing flow blockage in large-scale turbine arrays. In our large eddy simulations, we solve the filtered incompressible Navier-Stokes equations and continuity equation for a neutral pressure-driven ABL with a single inflow direction, as described in Chapter 3 Section 3.2.1. The viscous forces are neglected as we consider high Reynolds number atmospheric flows and we do not include thermal stratification or large-scale rotational effects. The streamwise periodic boundary conditions are negated by employing the concurrent precursor method to achieve realistic turbulent inflow conditions 62]. We follow this widely-accepted approach as it gives meaningful insight into atmospheric flow dynamics and allows us to study the effect of atmospheric turbulence intensity, without changing, for example, the capping inversion height. In this work, we consider onshore and offshore $\mathrm{ABL}$ conditions using the roughness heights $z_{0} / H=10^{-4}$ and $z_{0} / H=10^{-6}$, which correspond to a turbulence intensity of $12.1 \%$ and $7.5 \%$ at hub- 
height, respectively 22,84$]$.

The turbine forcing is generated using the actuator disk method described in references [18, 62 107$]$. We selected this actuator disk approach because it allows us to study blockage effects of extended wind farms in large eddy simulations. This method is widely used and provides valuable insight when the thrust coefficient is lower than the Betz limit $\left(C_{T}=8 / 9 ; a=1 / 3 ; C_{T}^{\prime}=2\right)$ 18 23,66 . These simulations are computationally demanding due to the long time-averaging that is required. Therefore, it is worth mentioning that the presented computations would not be feasible using an actuator line model [66 6108,109$]$ or an advanced actuator disk model [110, 111] as these models require a higher resolution to capture the turbine-specific features accurately. For example, Troldborg and Forsting [112] showed that actuator disk and actuator line model simulations capture the induction region in front of turbine with similar accuracy because the signature of individual blades is lost after time-averaging. Besides, Branlard and Forsting [102] showed that when $C_{T}$ is low, the velocity predictions agree well in the induction region obtained using an actuator disk model and a vortex cylinder model.

Turbulent statistics are gathered from the simulations as follows. Data is generated at each time step and once the simulations reach a statistically stationary state (after approximately 48 flow-through times), time-averaging is performed over roughly 70 subsequent flow-through times. Then, the results are normalized with the horizontallyaveraged incoming velocity of the inflow domain at hub-height so that the simulations are comparable. Nevertheless, some statistical uncertainty remains and is represented by the error bars. In Figure 4.1 the statistical uncertainty is visible by variations in the wind speed at various spanwise locations; however, as we consider spanwise periodic cases, these variations do not affect the presented findings.

\subsubsection{Simulation parameters}

This study compares a stand-alone turbine, a spanwise-infinite row of turbines, and a wind farm with eight rows, as depicted in Figure 4.1. Most importantly, we are interested in how the blockage effect depends on the average inter-turbine spacing and wind farm layout. Therefore, we consider multiple turbine array configurations varying the streamwise $\left(S_{x}\right)$ and spanwise $\left(S_{y}\right)$ spacing, which are made dimensionless using the turbine diameter, i.e. $s_{x}=S_{x} / D$ and $s_{y}=S_{y} / D$. For the rows, the spanwise spacing is varied between $s_{y}=1.26$ and $s_{y}=7.85$, which covers the range considered in previous studies $[98,113$. We also note that wind turbine arrays built alongside major roads can have a relatively small spanwise turbine spacing; see for example the Brazilian 'Delta 5 I" wind farm near Paulino Neves and the wind farm near "Sao Bento do Norte" in which the spanwise turbine spacing is roughly $2.5 \mathrm{D}$. In addition, to compare with the findings of Segalini 
and Dahlberg [105, we consider ten wind farm cases with spanwise spacing in the range $s_{y}=1.57$ to $s_{y}=3.93$ and streamwise spacing in the range $s_{x}=1.96$ to $s_{x}=7.85$. We use turbines with a hub-height $z_{h}=100 \mathrm{~m}$ and diameter $D=100 \mathrm{~m}$ which have a constant thrust coefficient $C_{T}=3 / 4$ (or $C_{T}^{\prime}=4 / 3$ ). We assign this value because this work originated from previous research in which $C_{T}=3 / 4$ was also used [78]. We note that modern commercial turbines have slightly higher $C_{T}$ and we show in Chapter 5 that the thrust coefficient does not significantly affect the nature of the observed blockage effect, but only its magnitude. We select eight rows $\left(N_{T, x}=8\right)$ for the wind farm case because as per the empirical results of Segalini and Dahlberg [90], blockage should be relatively insensitive to changing the number of rows past three, which we also confirmed by comparing simulations with 2,4 and 8 rows.

The turbines are placed in a sufficiently large domain with lengths $L_{x}, L_{y}, L_{z}=$ $(4 \pi, \pi, 1.0)$ discretized uniformly by $N_{x}, N_{y}, N_{z}=(512,128,128)$ grid points in the streamwise, spanwise, and vertical direction, respectively. The domain lengths are made dimensional by multiplying by the domain height $H=1000 \mathrm{~m}$, which is typical for a neutral ABL [18]. Also, we have observed previously that increasing the domain height does not significantly influence the results [114]. In our simulations, we follow the recommendations set by $\mathrm{Wu}$ and Porté-Agel [79], who showed that one needs 8 points along the turbine diameter in the vertical direction and 5 points along the turbine diameter in the spanwise direction. The (first) row of turbines is consistently placed at $x=5 \mathrm{~km}$ $(x / D=50)$ to ensure that the preceding induction region is adequately captured. This choice is based on our observation that induction effects are negligible (i.e. smaller than $0.1 \%$ of the inflow velocity) beyond $x / D=-12$ in front of the first row for all cases considered in this study. The fringe region at the end of the domain covers $9 \%$ of the streamwise domain length $L_{x}$. Therefore, the distance between the wind farm and the fringe region depends on the considered turbine layout. To ensure that the domain is long enough, we performed a simulation where the streamwise domain length was doubled from $L_{x}=4 \pi$ to $L_{x}=8 \pi$ for the wind farm layout in which we observed the strongest blockage ( $s_{y}=1.57, s_{x}=1.96$ ). In this case, the power output of the first row of the wind farm is the same within $0.01 \%$, confirming that the downstream domain length is sufficient. In Section 4.3.3 we also examine the effect of the spanwise extent of the array on the blockage effect in our numerical experiment as real wind farms are not spanwise-periodic. 


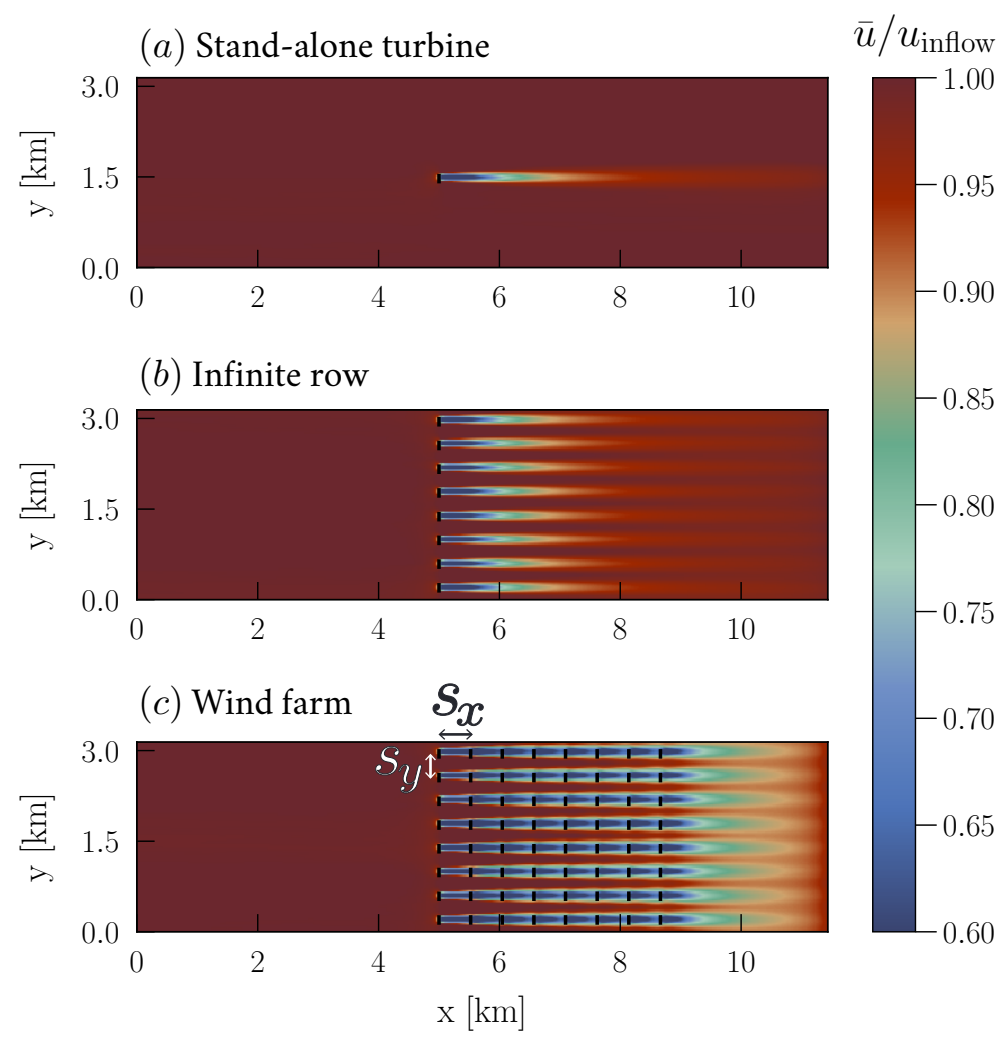

Figure 4.1: Time-averaged streamwise velocity normalized by the inflow velocity at hub-height in offshore conditions $\left(z_{0} / H=10^{-6}\right)$ shown for (a) a stand-alone turbine, (b) an infinite solitary row of turbines $\left(s_{y}=3.93\right)$, and (c) a wind farm with eight rows in the downstream direction $\left(s_{y}=3.93, s_{x}=5.24\right)$.

\subsection{Results and discussion}

We analyze flow blockage for multiple turbine arrays by comparing variations in the relative power output and the induction region. First, we investigate spanwise blockage effects caused by close spanwise turbines by comparing the performance of an infinite row of turbines to a stand-alone turbine. Next, we examine streamwise blockage caused by downstream turbines by comparing the performance of the first row of a wind farm to both a stand-alone turbine and a solitary infinite row. Finally, we study the effect of the array's spanwise extent as well as the turbine hub-height. 


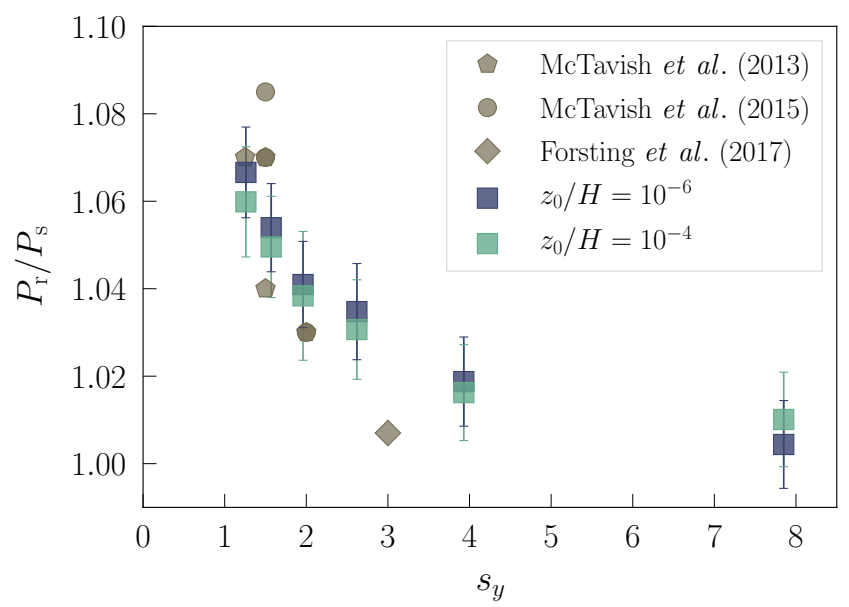

Figure 4.2: The average power of a wind turbine in an infinite row relative to the average power of a stand-alone turbine as a function of dimensionless spanwise spacing $s_{y}$. Data is plotted for onshore (green) and offshore (blue) surface friction conditions, which are represented by roughness scales $z_{0} / H=10^{-4}$ and $z_{0} / H=10^{-6}$, respectively. The error bars are based on the standard deviation of the temporal power variation. Results from experiments by McTavish et al. 98,113 and simulations by Forsting et al. [99] are shown for comparison.

\subsubsection{Spanwise blockage effects in an infinite row}

Figure 4.2 shows the average power output of a turbine in an infinite row relative to that of a stand-alone turbine as a function of spanwise spacing for onshore and offshore conditions. We observe that the turbine power output in an infinite row is higher than a stand-alone turbine when the spanwise spacing is small. If turbines are close together, the flow deflected by one turbine, passes through the rotor-swept area of the neighboring turbines, causing them to be more productive than in isolation. The relative benefit of the blockage effect diminishes as the spacing increases. As a result, at large spacing $\left(s_{y}=7.85\right)$, the power of a turbine in an infinite row approaches that of a stand-alone turbine. Interestingly, the results are only marginally influenced by onshore and offshore surface friction conditions, varying less than $1 \%$. Furthermore, the figure shows that our large eddy simulations agree well with the wind tunnel measurements by McTavish et al. 98 113] and RANS results by Forsting et al. [99]. We note that the value found by Forsting et al. [99. may be lower because we consider an infinite row and therefore, we briefly address the influence of the spanwise row extent in Section 4.3.3

Decreasing the spanwise spacing within an infinite row is expected to intensify the induction region preceding the turbines. Figure 4.3 shows the time-averaged streamwise 

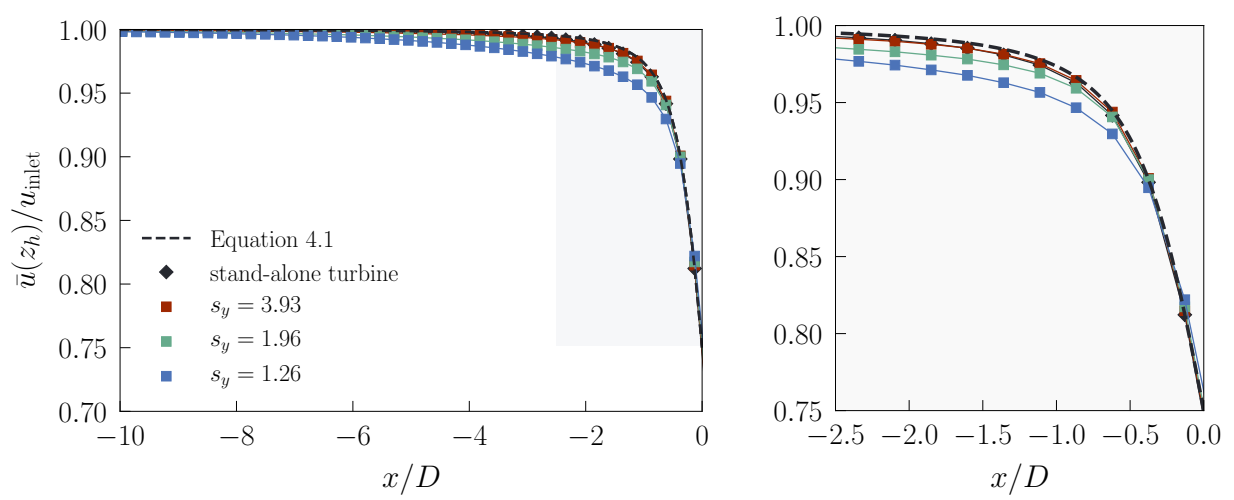

Figure 4.3: The time-averaged streamwise velocity at hub-height along the centerline preceding each turbine normalized by the inlet velocity as a function of the position upstream (turbines are located at $x / D=0$ ) shown for a stand-alone turbine and selected infinite row cases in offshore conditions. The dashed line represents the theoretical prediction for induction in front of a single turbine (Equation 4.1 .

velocity at hub-height and along the centerline of a turbine preceding the turbine(s) normalized by the velocity at the entrance $u_{\text {inlet }}$. We observe that the large eddy simulation for the single turbine agrees excellently with the theoretical prediction (Equation 4.1], confirming that our simulations capture the induction effect accurately and that the induction is not significantly influenced by the atmospheric turbulence intensity. Figure 4.3 also shows that for the infinite row cases where the results are averaged over all turbines columns, the induction is stronger than for the stand-alone turbine case. As expected, the velocity deficit is more pronounced and extends farther upstream when the spanwise spacing decreases. In fact, for $s_{y}=1.26$, the velocity is $0.1 \%$ of the incoming wind speed at $x=-12 D$ in front of the turbines. At first glance, the stronger induction for smaller $s_{y}$ seems to contradict the aforementioned power production increase due to spanwise blockage for small $s_{y}$. However, Figure 4.3 reveals that the wind speed just in front of the turbines $(x / D=-0.5$ to $x / D=0)$ increases with decreasing $s_{y}$. We believe this behavior is caused by a favorable pressure gradient created directly in front of the turbine, or in other words, spanwise flow deflection becomes visible for spanwise closely-spaced turbines. Overall, this enhanced induction effect should be considered when analyzing field data within this range. 


\subsubsection{Blockage effects in large-scale wind farms}

We investigate flow blockage caused by downstream turbines by comparing the performance of the first row of a wind farm to a stand-alone turbine and a solitary infinite row of turbines. Although blockage could affect all rows of the wind farm, the effect is most evident in the first row. Figure 4.4 a) shows the average power output of a turbine in the first row of a wind farm compared to a stand-alone turbine as a function of the spanwise spacing $s_{y}$. We observe that for large streamwise spacing $s_{x}$, the trend is similar to the corresponding solitary row case (see Figure 4.2. However, when the downstream turbines are closer $\left(s_{x}=1.96\right)$, the trend differs, and the power output of turbines in the first row is lower than a stand-alone turbine. Therefore, close downstream turbines can negatively impact the performance of upstream turbines. Figure $4.4 \mathrm{~b}$ ) confirms that the turbine power production decreases when $s_{x}$ decreases for each considered $s_{y}$. However, the data does not collapse as a function of $s_{x}$.

Clearly, both the spanwise and streamwise blockage impact the turbine performance differently. The findings of Segalini and Dahlberg [90] suggest that the global blockage effect depends on the wind turbine density. Therefore, in Figure $4.5(\mathrm{a})$, we compare the average power output of a turbine in the first row of a wind farm to that of a stand-alone turbine as a function of the dimensionless mean turbine spacing $s=\sqrt{s_{x} s_{y}}$. However, we do not observe a universal trend, indicating that wind farm blockage is a more complicated phenomenon and that the specific combination of $s_{x}$ and $s_{y}$ (i.e. the wind farm layout), determines the blockage effect.

However, it should be emphasized that the wind tunnel experiments of Segalini and Dahlberg 90 normalized the results using the performance of a relatively solitary turbine row. Therefore, in Figure 4.5 b), we show the data normalized by the corresponding solitary row. For each layout, the first row of the wind farm has a lower power output than a turbine in the corresponding solitary row. In agreement with Equation 4.2 we find that our results collapse when normalized by the performance of the corresponding solitary row case and become independent of the wind turbine layout as this essentially normalizes out the spanwise blockage effects. Additionally, these simulations were executed for both onshore and offshore conditions, and we observe that blockage is not significantly dependent on the surface roughness conditions. As we observe a similar trend as Segalini and Dahlberg [90], which was obtained using uniform inflow, we conclude that the atmospheric turbulence intensity has a marginal effect on the blockage, suggesting that blockage is mainly caused by mass conservation and pressure effects.

Wind is deflected over wind farms $30,115,116$, and therefore blockage reduces the power production of the first wind farm row compared to the corresponding solitary row. To demonstrate this, we analyze the spanwise-averaged vertical velocity for three different wind farm layouts in Figure 4.6 In general, we observe that the flow is deflected 
(a)

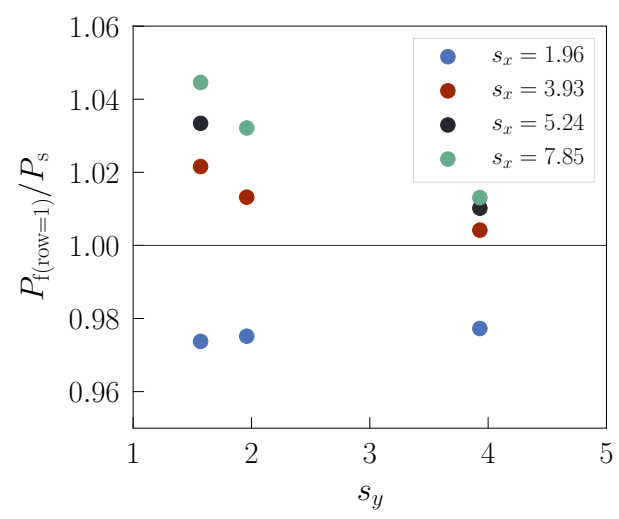

(b)

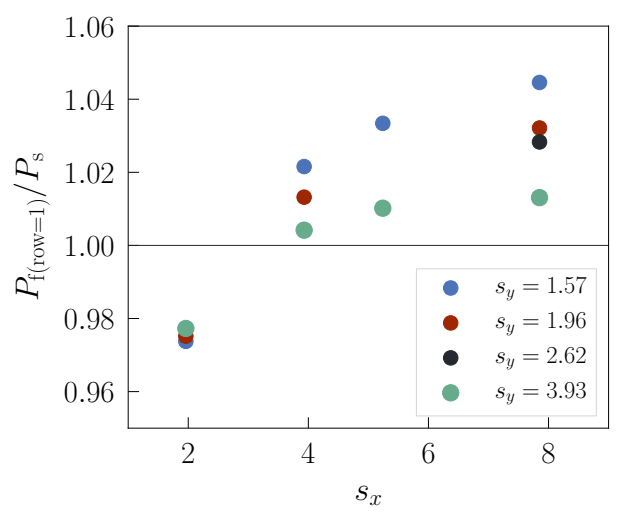

Figure 4.4: The average power output of a turbine in the first row of a wind farm relative to the average power output of a stand-alone turbine in offshore conditions as a function of the dimensionless (a) spanwise turbine spacing $s_{y}$ and, (b) streamwise turbine spacing $s_{x}$.

(a)

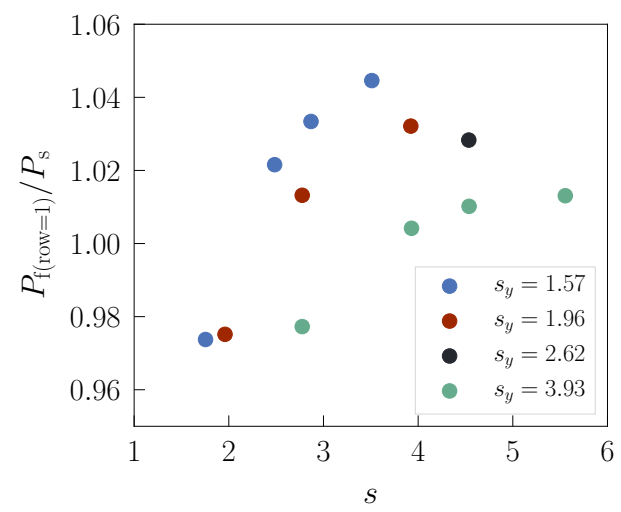

(b)

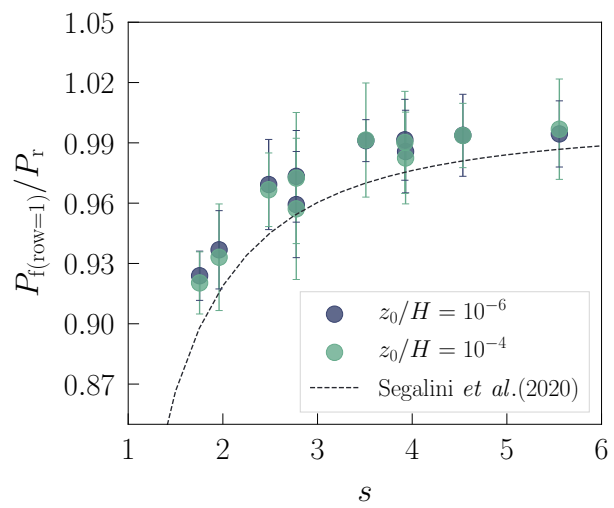

Figure 4.5: The average power output of a turbine in the first row of a wind farm compared to the power production of (a) a stand-alone turbine and (b) the corresponding solitary row with the same spanwise spacing, as a function of the dimensionless geometrical mean turbine spacing $s=\sqrt{s_{x} s_{y}}$. In panel (b) the error bars are based on the standard deviation of the temporal power variation and our results are compared with Equation 4.2 
(a) $s_{y}=3.93, s_{x}=5.24$

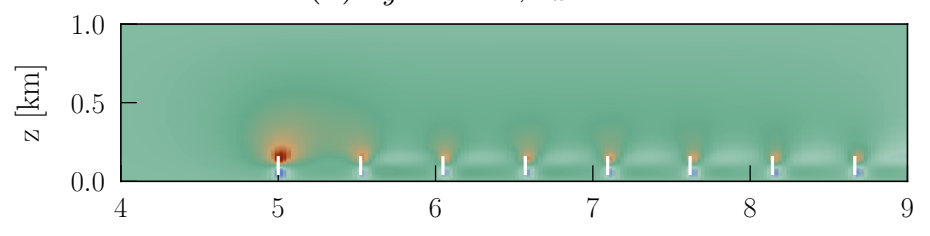

(b) $s_{y}=1.57, s_{x}=5.24$

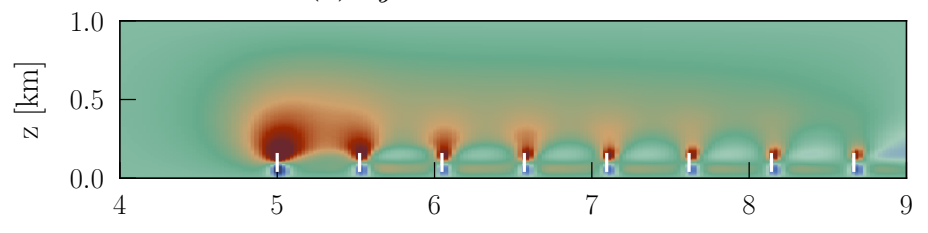

(c) $s_{y}=1.57, s_{x}=1.96$

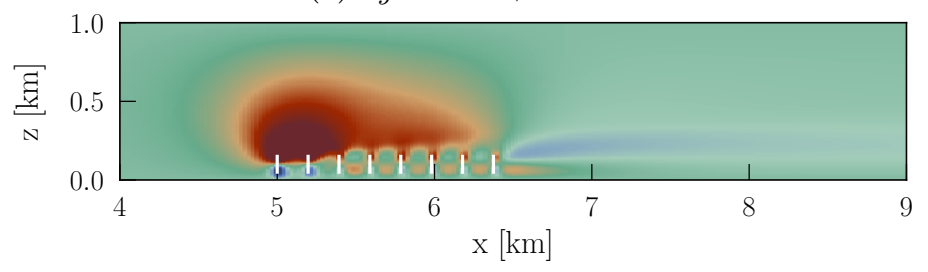

$\langle\bar{w}\rangle_{y} / u_{\text {inflow }}$

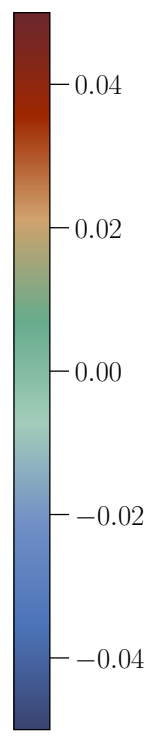

Figure 4.6: The time- and spanwise-averaged vertical velocity for three different wind farm configurations in offshore conditions.
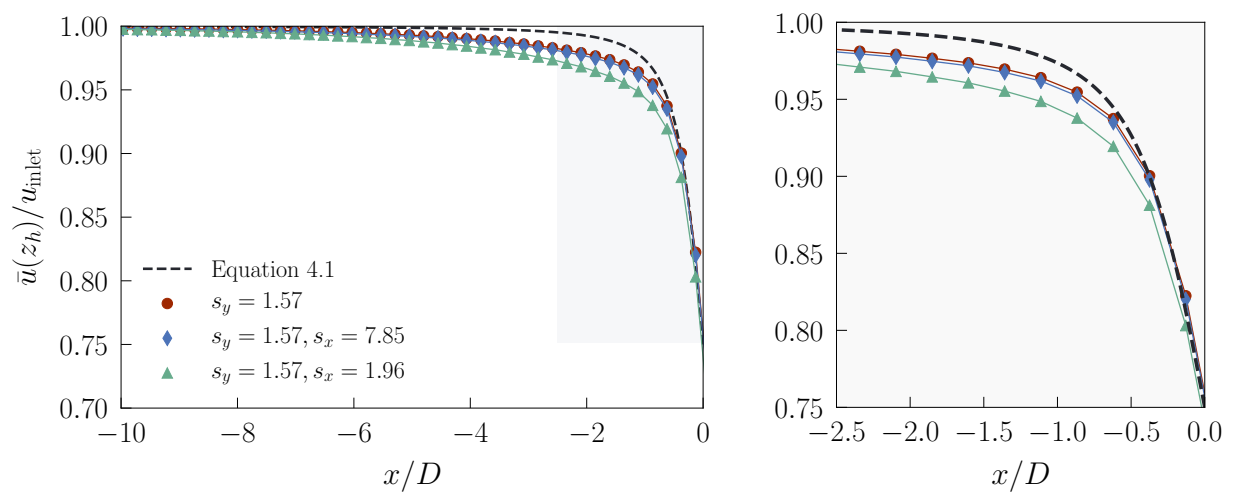

Figure 4.7: The time-averaged streamwise velocity at hub-height along the centerline preceding each turbine normalized by the inlet velocity as a function of the position upstream for two selected wind farm cases and an infinite row case for offshore conditions. The dashed line represents the theoretical prediction for induction in front of a single turbine (Equation 4.1. 


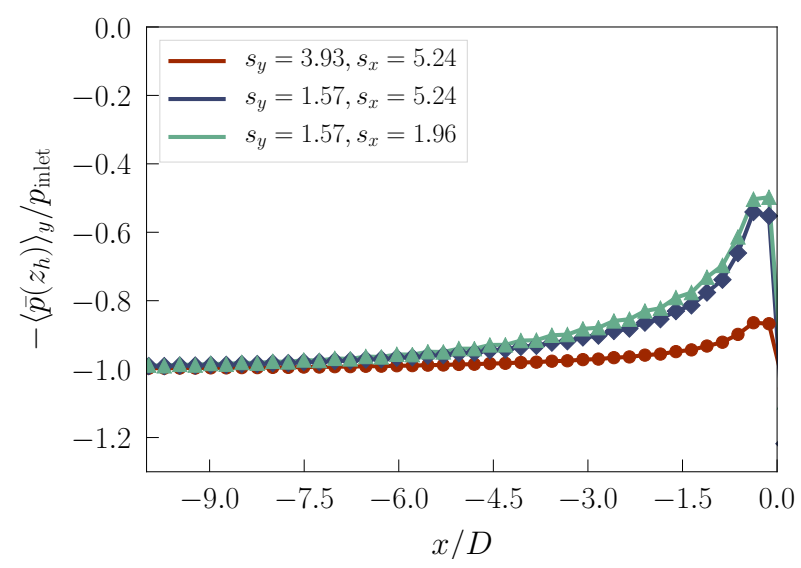

Figure 4.8: The time- and spanwise-averaged pressure perturbation at hub-height normalized by the inlet value for three wind farm layouts in offshore conditions.

around the turbine, accelerating above and below the actuator disk. Comparing Figures 4.6 a) and 4.6 (b), where only the spanwise spacing is reduced, reveals that the upward movement of the air at the entrance of the wind farm becomes more pronounced when the spanwise spacing decreases. In Figure 4.6(c), the turbine density is increased further by also reducing the streamwise spacing. In this case, the upward flow deflection at the wind farm entrance is even stronger. This observation supports the conclusion that the flow over the wind farm increases when the inter-turbine spacing decreases.

In Figure 4.7 we compare the centerline streamwise velocity at hub-height for two wind farms and the corresponding solitary infinite row with the same spanwise spacing. We observe that both wind farm cases induce a more pronounced velocity deficit than the row case. When the streamwise spacing is set to a commonly-employed separation $\left(s_{x}=7.85\right)$, the induction of the wind farm is similar but still slightly stronger than the corresponding infinite row case without downstream turbines. However, when $s_{y}=1.57$ and $s_{x}=1.96$, the induction region preceding the first turbine row extends up to $13 \mathrm{D}$ upstream before reaching $0.1 \%$ of the incoming velocity compared to $11 \mathrm{D}$ for the corresponding row case.

More wind is deflected over the wind farm and induction is enhanced when the turbines are placed closer together due to a change in the pressure gradient. Figure 4.8 shows the spanwise-averaged pressure field for three different wind farm layouts as a function of the position upstream. First, we observe the inherent adverse pressure gradient preceding each wind farm which characterizes the induction zone where wind is slowed. Second, the figure shows that the streamwise pressure gradient becomes stronger 
when the inter-turbine spacing decreases. The amplified adverse pressure gradient further slows the wind at hub-height and this effect is visible in Figure 4.7 Overall, both spanwise and downstream neighbors significantly affect the flow preceding large-scale wind farms, and therefore, it is imperative to consider blockage in wind farm design.

\subsubsection{Effects of spanwise row extent}

Here, we briefly address the effect of the row length $L_{r}$ on wind farm blockage. The results are presented as a function of the ratio $L_{r} / L_{y}$ (refer to Figure 4.9 (a)), where the domain length remains constant $\left(L_{y}=\pi\right)$. Specifically, we compare the infinite row cases $\left(L_{r} / L_{y}=1.0\right)$ to various finite row cases. The spanwise spacing remains constant $\left(s_{y}=1.57\right)$ while the row is shortened by decreasing the number of turbines such that $L_{r} / L_{y}=0.8,0.5,0.2$, corresponding to $N_{T}=16,10,4$, respectively. In Figure 4.9 (b), the average power output of a turbine in each row is compared to that of a stand-alone turbine. As expected, we observe that the normalized power output decreases with the row length because air is permitted to flow around the array, reducing the beneficial spanwise blockage effect. And yet, even when finite, a significant benefit from the blockage effect is observed as the average turbine within a row is more productive than a stand-alone turbine for each case. Separately, we also verified that the power ratio remains the same within $0.02 \%$ when the spanwise domain length is doubled from $L_{y}=\pi$ to $L_{y}=2 \pi$ but $L_{r} / L_{y}=0.5$ remains constant, further supporting that the selected domain length is sufficient and that the parameter of interest is the spanwise extent of the row within the domain. Additionally, we performed simulations with $s_{y}=1.26$ and $N_{T}=25$ turbines in both a $L_{y}=\pi$ to $L_{y}=2 \pi$ domain. We find that increasing the domain size while $L_{r}$ remains constant reduces the blockage effect from 1.067 to 1.039 , suggesting that the spanwise blockage effect reduces by a factor of less than 2 when the domain size is doubled. This confirms that, although spanwise blockage is stronger in spanwise-infinite turbine arrays, blockage still occurs in spanwise-finite wind farms.

Figure 4.9 (c) compares the average power output of a turbine in the first row of a wind farm to that of a stand-alone turbine for each $L_{r} / L_{y}$. We observe that the average turbine in the first row of the spanwise-infinite wind farm produces less power than a stand-alone turbine, and when the rows are finite, the relative power decreases further. In Figure 4.9 d), we show that by normalizing the performance of the first wind farm row by the performance of the corresponding solitary row, the results become independent of the spanwise wind farm extent, confirming our earlier agreement with Segalini and Dahlberg $[90]$ in Figure 4.5 . However, as previously stated, it is crucial to acknowledge that our results reveal that wind farm blockage depends on the turbine layout as the universal trend is only recovered when normalizing with the corresponding row case. 
We emphasize that the results do not show a universal trend when normalized with the performance of a stand-alone turbine.

(a)

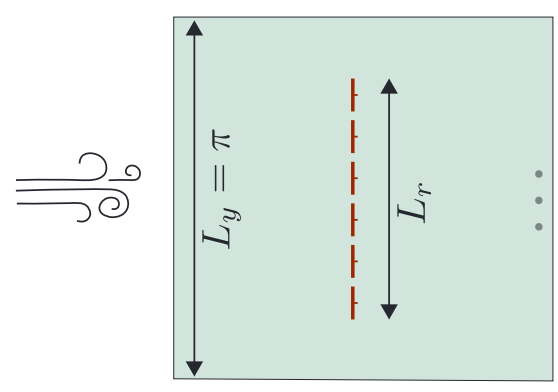

(c)

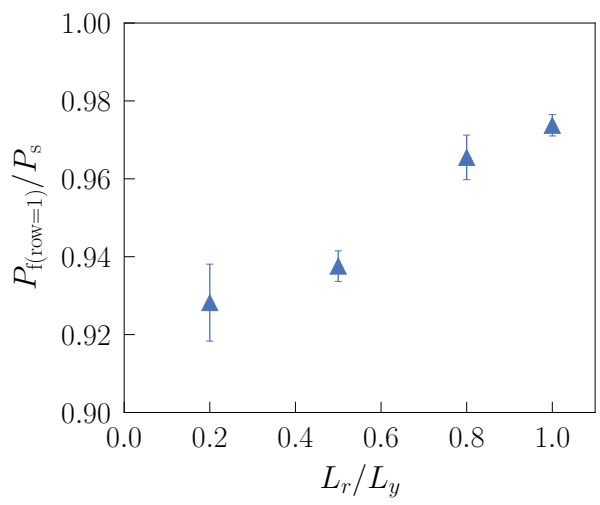

(b)

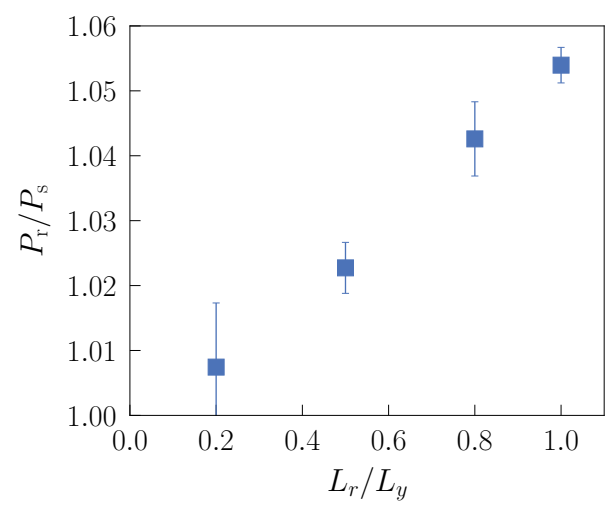

(d)

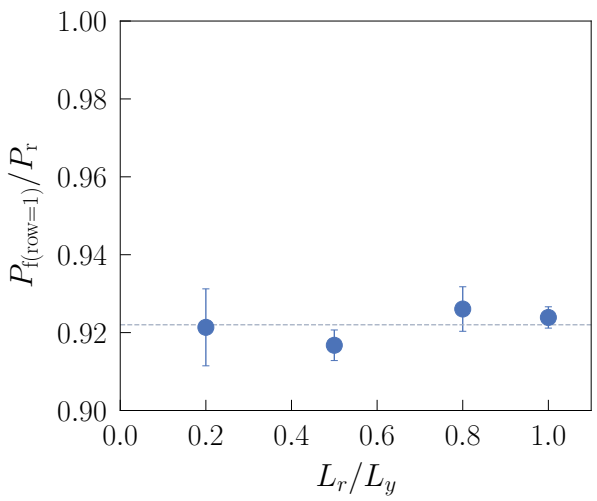

Figure 4.9: (a) Schematic depicting the spanwise domain length $L_{y}=\pi$ and the turbine row length $L_{r}$. (b) The average power output of a turbine in a row $\left(s_{y}=1.57\right)$ relative to a stand-alone turbine. (c) The average power output of a turbine in the first row of a wind farm $\left(s_{y}=1.57, s_{x}=\right.$ 1.96) relative to a stand-alone turbine, and (d) relative to a solitary row $\left(s_{y}=1.57\right)$ where the dashed line is the average value. These results are generated using offshore conditions. 


\subsubsection{Effects of turbine hub-height}

We expect that blockage will be influenced by how close the turbines are to the surface because this will affect the airflow under the turbines. Therefore, in Figure 4.10 we present the average power output of a turbine in the first row of a wind farm relative to the corresponding row case for four different hub-heights: $z_{h}=75 \mathrm{~m}, 100 \mathrm{~m}, 125 \mathrm{~m}, 150 \mathrm{~m}$, while the rotor diameter remains constant $(D=100 \mathrm{~m})$. We investigate the effect of hub-height for the most dense wind farm configuration considered in this study because the larger magnitude of the blockage effect will then be more sensitive to changes. We observe that the effect of hub-height is marginal, but measurable, as the relative power increases as $z_{h}$ increases. When the turbines are farther from the surface, blockage is reduced because more air can flow underneath the turbines, and the relative power of the first row increases. The influence of the hub-height appears to diminish as $z_{h}$ increases. Nonetheless, the blockage effect still impacts the performance of the first row when $z_{h}=150 \mathrm{~m}$, with $100 \mathrm{~m}$ between the bottom of the rotor-swept area and the surface. The fact that turbines should not be placed too closely to the ground is well-accepted, but also, increasing the hub-height will not eliminate blockage effects.

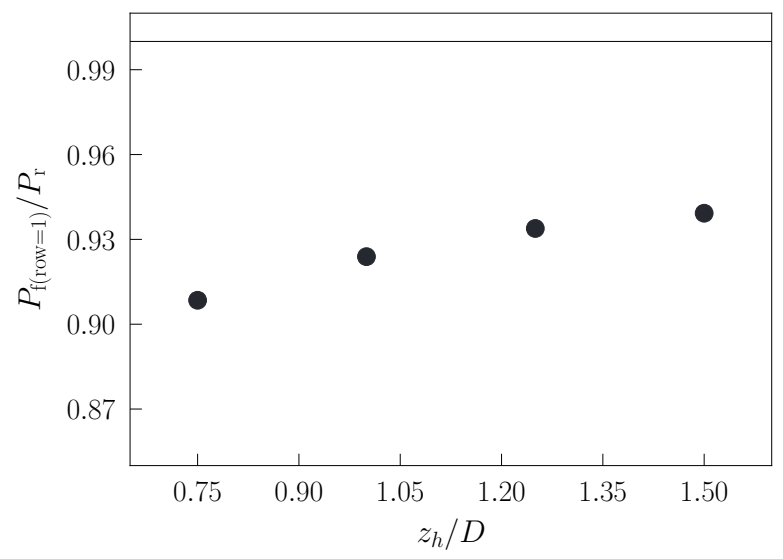

Figure 4.10: The average power output of a turbine in the first row of a wind farm $\left(s_{y}=\right.$ $\left.1.57, s_{x}=1.96\right)$ compared to the power of the corresponding solitary row $\left(s_{y}=1.57\right)$ as a function of the dimensionless hub-height. 


\subsection{Conclusions}

We used high-fidelity large eddy simulations to study blockage effects in large-scale wind farms. We determined that blockage impacts the power production of turbines when they are placed close together by exploring an encompassing set of wind farm configurations, focusing on spanwise and streamwise blockage effects. Specifically, we compared the performance of a stand-alone turbine, a spanwise-infinite row of turbines, and a wind farm with eight rows. In all cases, we established that the blockage effect in a neutral ABL is not dependent on the atmospheric turbulence intensity at hub-height when comparing offshore and onshore conditions but is highly dependent on the dimensionless turbine spacing and wind farm layout. Therefore, our results provide further support for the view that the blockage effect does not depend on the turbulent mixing, but on the pressure distribution created in the wind farm 90 .

We observed that closely-spaced spanwise turbines are more productive due to spanwise blockage as the average power production of a turbine in an infinite row is higher than a stand-alone turbine. Instead of passing around the turbine, the flow is deflected into the rotor-swept area of neighboring turbines. Consequently, this effect increases as the spanwise spacing decreases. At more commonly-employed spanwise spacing, this effect is minimal, but at small spanwise spacing, our results are consistent with the available literature $98,99,113$. The spanwise blockage effect is enhanced by the fact that we use spanwise-infinite rows, however, we also verified that the effect is still present when the rows are finite.

By comparing the average performance of the first row of a wind farm to a standalone turbine, it becomes clear that streamwise and spanwise blockage differ and have competing effects. We observed that placing turbines downstream hinders upstream turbines as the average power of a turbine in the first row of a wind farm was lower than that of a turbine in a corresponding solitary row. Essentially, the flow is inclined to pass over the dense array as opposed to through it, which we confirmed by analyzing the vertical velocity at the entrance of the wind farm. We also found that increasing the turbine hub-height could allow more air to flow below the turbines, reducing the blockage effect. However, the influence of the turbine hub-height is subtle and diminishes as the hub-height increases. In agreement with the wind tunnel experiments by Segalini and Dahlberg [90], we observed that the performance of the first wind farm row normalized by the corresponding solitary row shows a universal curve when plotted as function of the average inter-turbine spacing. The fact that our values are higher is expected considering the difference in thrust coefficient 114]. However, we did not find a universal trend when the performance of the first wind farm row is normalized by the performance of a stand-alone turbine. From this, we conclude that the specific wind farm layout influences 
wind farm blockage.

We also established how wind farms can affect the flow in the induction region preceding the array. We observed that a closely-spaced infinite row induces a more prominent, farther-reaching velocity deficit than a stand-alone turbine. Likewise, the induction was even more pronounced for a wind farm, which has also been observed for other wind farm configurations $91,101,102]$. In summary, the induction region becomes more pronounced as the spacing between the turbines decreases, and in the densest case we considered $\left(s_{x}=1.96, s_{y}=1.57\right)$, is detectable $(0.1 \%$ of the inflow velocity) up to $13 D$ in front of the first row. Accordingly, we observed that the adverse pressure gradient, which slows the wind in the induction region, is exacerbated when the turbine density increases. These upstream effects require more research and should be considered when planning wind farms.

In this study, we showed that cumulative blockage effects depend on the wind farm layout and further studies are required to address in what way this could be modeled. Future work can be enhanced by considering the effect of finite rows and the wind farm layout in more detail. We showed that blockage effects in a simplified ABL configuration are comparable to what is observed in uniform inflow configurations by Segalini and Dahlberg 90, 105]. However, even more realistic boundary layer conditions, which involve the Coriolis force and thermal stratification still need to be considered. Moreover, blockage is expected to increase in a stable boundary layer and may interact with other physical phenomena such as gravity waves $96,117,118$. Regardless, we have established that it is important to be aware of wind farm blockage effects and that the performance of the first wind farm row is far from a perfect reference. 


\section{Chapter 5: EFFECT OF THE THRUST COEFFICIENT ON BLOCKAGE IN CLOSELY-SPACED TURBINE ARRAYS}

The performance of a wind turbine within an array is not necessarily equivalent to the performance of a free-standing turbine because the presence of the neighboring turbines influences the incoming flow. However, insight into these blockage effects is still lacking. Here, we use large eddy simulations to investigate how the blockage effect depends on the turbine thrust coefficient $C_{T}$. We consider three main scenarios: a free-standing turbine, a closely-spaced spanwiseinfinite row of turbines, and an entire wind farm with a high turbine density. We observe that the power production of turbines in the row increases approximately linearly with $C_{T}^{\prime}$ when compared to the production of a free-standing turbine. Here, $C_{T}^{\prime}=C_{T} /(1-a)^{2}$ where $a$ is the induction factor. In contrast, the power output of turbines in the first row of a wind farm decreases approximately linearly with $C_{T}$ when compared to the production of turbines in a corresponding solitary row.

Adapted from publication: Jessica M. I. Strickland and Richard J. A. M. Stevens, Effect of thrust coefficient on the flow blockage effects in closely-spaced spanwise-infinite turbine arrays, J. Phys. Conf. Ser. 1618, 062069 (2020). 


\subsection{Introduction}

Accurately modeling wind farm performance is becoming more important as global energy demands and investment in wind energy grow at an unprecedented rate [23, 66]. The main goal of most wind farm models is to obtain accurate turbine power production estimates. Currently, most models focus on the physics downstream of the turbines. For instance, wind turbine wake models typically impose wake deficits downstream to reflect the extracted kinetic energy [23]. As modeling wake effects and wake interactions in large wind farms is still quite challenging, it is still common practice to normalize the power production of downstream turbine rows with the power production of the first row. By normalizing data using the first row, it is implicitly assumed that the first row is always performing as it would in isolation without downstream turbines.

Although this assumption is appealing, it is not correct. Downstream turbines can influence upstream turbines as it follows from actuator disk theory that the flow upstream of a turbine is affected by its presence [92]. Due to blockage effects, the performance of a turbine in the first row of a wind farm is not necessarily equal to that of a turbine placed in isolation. Given that the power output of the first turbine row is often used as a reference, accounting for blockage effects and understanding the relevant parameters is crucial to arrive at reliable estimates for the power production of large wind farms.

Previous studies have shown that blockage can both increase and decrease the power output of neighboring turbines and have highlighted the importance of understanding the effects for a wider range of conditions. Previous experiments and simulations have shown that the power output of laterally closely-spaced turbines can be higher than that of isolated turbines $\left.\begin{array}{l|l|l}98 & 113 & 119\end{array}\right]$. This increase is due to a Venturi effect, i.e. the flow is accelerated between the closely-spaced turbines causing their power output to increase significantly. The increased power production is essentially a blockage or confinement effect due to which additional wind is forced through the turbine-swept area.

For large wind farms another effect is observed. Segalini and Dahlberg [90] and Bleeg et al. [101] namely show that for large wind farms, the flow velocity at the first row can be significantly reduced as the wind is deflected over and around the entire wind farm. As a result, turbines in the first row will produce less energy than they would without the downstream turbines being present. In this study, we use large eddy simulations to better understand the effect of the turbine thrust coefficient on the flow blockage effect and to ultimately provide more accurate predictions of its impact on the wind farm performance.

This chapter is divided into three main sections to follow. Section 5.2 describes the modeling details and case parameters and characteristics. Next, the results are presented and discussed in Section 5.3 addressing the dependence of the blockage effect on the thrust coefficient. Finally, the major conclusions are summarized in Section 5.4 


\subsection{Numerical framework}

\subsubsection{Large eddy simulations}

Our large eddy simulations for a neutrally-stratified atmospheric boundary layer (ABL) are governed by the filtered incompressible Navier-Stokes equations without buoyancy, system rotation, or other effects.

$$
\begin{gathered}
\partial_{t} \tilde{u}_{i}+\partial_{j}\left(\tilde{u}_{i} \tilde{u}_{j}\right)=-\partial_{i} \tilde{p}^{*}-\partial_{j} \tau_{i j}-\delta_{i 1} \partial_{1} p_{\infty} / \rho+f_{i}, \\
\partial_{i} \tilde{u}_{i}=0,
\end{gathered}
$$

where $\tilde{u}$ is the filtered velocity field and $\tilde{p}^{*}$ is the filtered modified pressure such that $\tilde{p}^{*}=\tilde{p} / \rho+\tau_{k k} / 3-p_{\infty} / \rho$. The sub-grid scale stresses are represented in the term $\tau_{i j}$. The deviatoric part, $\tau_{i j}-\delta_{i j} \tau_{k k} / 3$, is parametrized using a eddy-viscosity sub-grid scale model. Specifically, we employ the Lagrangian scale-dependent dynamic sub-grid model and $\tau_{k k} / 3$ is incorporated into the modified pressure. The turbine forcing is represented by $f_{i}$ and the viscous forces are neglected as we consider high Reynolds number atmospheric flows.

The governing equations are solved as follows. The time integration is performed using a second-order accurate Adams-Bashforth scheme. We consider periodic boundary conditions in the horizontal directions, as a pseudo-spectral discretization is used. The top boundary is characterized by a no-stress condition with the vertical velocity set to zero. The stress at the bottom boundary is related to the velocity of the first grid point relying on the classic logarithmic law for turbulent wall-bounded flow 67.68. In our cases the surface roughness characterizing the logarithmic law is set to a typical offshore value: $z_{0}=0.001 \mathrm{~m}$ [59].

A concurrent precursor method is used to generate realistic ABL inflow conditions. In this way, two domains are considered simultaneously: a fully developed neutral ABL which is used to create realistic inflow for a second domain that contains wind turbines. The turbulent inflow data is sampled from the ABL simulation and is introduced at the entrance of the target wind farm domain via a fringe region. Further details about the simulation approach can be found in reference [62.

The actuator disk model is used to represent the turbines $18,62,107]$. This method includes the turbines as disks that exert a total thrust force

$$
F_{T}=-\frac{1}{2} \rho C_{T} U_{\infty}^{2} A,
$$

where $\rho$ is the density of air, $A=\frac{\pi}{4} D^{2}$ is the area of the rotor disk, and $C_{T}$ is the thrust coefficient. Equation 5.3 requires the undisturbed upstream velocity $U_{\infty}$. However, 
identifying the upstream velocity can be difficult in wind farm simulations. To circumvent this issue, the use of the velocity at the turbine location is preferred [18,74]. This is achieved by using actuator disk theory to relate the velocity across the disk $U_{D}$ with the disk-based thrust coefficient $C_{T}^{\prime}$ which is defined as per the following relationship

$$
C_{T}^{\prime}=\frac{C_{T}}{(1-a)^{2}}, \text { where } a=\frac{U_{\infty}-U_{D}}{U_{\infty}} .
$$

The axial induction factor $a$ relates the velocity across the disk to the velocity upstream and $C_{T}=4 a(1-a)[71]$. In our simulations, each turbine is assigned the same $C_{T}^{\prime}$. This simplification is based on a reasonable assumption that the turbines will mostly operate in a regime where the thrust coefficient will be independent of wind speed [120]. Though the actuator disk model is not an exact representation of the turbines, this method is widely used in the literature and provides useful insight when the thrust coefficient is lower than the Betz limit $\left(C_{T}=8 / 9, a=1 / 3, C_{T}^{\prime}=2\right)$. By multiplying the drag force by the average velocity across the disk, we obtain an expression for the power produced by the turbines: $P=-F_{T} U_{D}$. Therefore, we calculate the power output of the turbines using the following expression.

$$
P=\frac{1}{2} \rho C_{T}^{\prime} U_{D}{ }^{3} \frac{\pi}{4} D^{2}
$$

Additionally, in our analysis we consider time-averaged values. We perform simulations for a sufficient period to ensure that the wind farm data is converged. Timeaveraging begins after 20 dimensionless time units (approximately 40 flow-through times) when the statistical stationary state is reached. The error bars are calculated using the standard deviation of the average power obtained from successive intervals of 20 time units after the start of time-averaging and following the general rules for the propagation of uncertainties. Also, we normalize the turbine power output for each simulation with the incoming flow at hub-height to accurately compare the performance data from different simulations.

\subsubsection{Simulation specifications}

We study three main layouts, as illustrated in Figure 5.1 and the details of the simulations are summarized in Table 5.1 First, to investigate flow blockage effects in laterally closelyspaced rows, we compare the performance of turbines in an infinite row to that of a free-standing turbine. Then, we investigate the effect of turbines placed downstream by comparing the first row of a spanwise-infinite wind farm with eight rows in the downstream direction, to that of a solitary row with the same spanwise turbine spacing. 
(a) Free-standing turbine

(b) Infinite row

(c) Wind farm

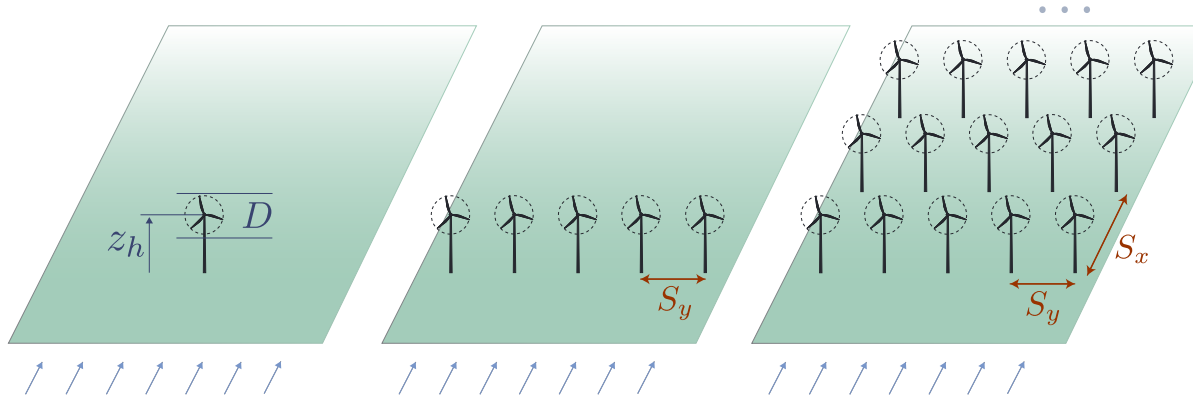

Figure 5.1: Schematic depicting the three main cases considered in this study: (a) a free-standing turbine (b) a solitary infinite row, and (c) a wind farm with eight rows in the downstream direction. The streamwise and spanwise spacing are made dimensionless using the turbine diameter $D$ : $s_{x}=S_{x} / D, s_{y}=S_{y} / D$. See Table 1 for a detailed overview of the cases considered.

Although the dimensionless streamwise $s_{x}$ and spanwise $s_{y}$ turbine spacing are also varied, we primarily focus on the dependence of the blockage effect on the turbine thrust coefficient in the current analysis by systematically varying $C_{T}^{\prime}$.

For all cases we consider the following fixed parameters. Both the turbine hub-height $z_{h}$ and diameter $D$ are $100 \mathrm{~m}$. The dimensionless domain size used is $L_{x}, L_{y}, L_{z}=$ $(4 \pi, \pi, 1.0)$, which is discretized on a grid with $N_{x}, N_{y}, N_{z}=(512,128,128)$ nodes in the streamwise, spanwise, and vertical direction, respectively. The domain size is made dimensionless using the height of the domain which is assigned as $H=1000 \mathrm{~m}$ unless otherwise specified, because this is a typical height of a neutral ABL [18]. The streamwise location of the (first) row of turbines is constant, $x / H=5.0$, to ensure there is ample space preceding the farm to observe the upstream influence of the wind turbines.

\begin{tabular}{llll}
\hline Case & $s_{y}$ & $s_{x}$ & $C_{T}^{\prime}$ \\
\hline Wind farm (8 rows) & 1.57 & $1.96,3.93,7.85$ & $1 / 6,1 / 3,2 / 3,1,4 / 3,5 / 3$ \\
Infinite row & $1.26,1.57$ & - & $1 / 3,2 / 3,1,4 / 3,5 / 3$ \\
Free-standing turbine & - & - & $1 / 3,2 / 3,1,4 / 3,5 / 3$ \\
\hline
\end{tabular}

Table 5.1: List of the simulated cases. The variables of interest are the dimensionless streamwise $s_{x}$ and spanwise $s_{y}$ spacing, and the disk-based thrust coefficient $C_{T}^{\prime}$ (refer to Equation 5.4). 


\subsection{Results and discussion}

Here we examine the impact of the turbine thrust coefficient on the blockage effect. First, we compare the power output of a turbine in a closely-spaced spanwise-infinite row to that of a free-standing turbine. Next, the performance of the first row of a wind farm is compared to both the corresponding row case and a free-standing turbine.

\subsubsection{Blockage effects for an infinite row of turbines}

Figure 5.2 shows the relative power output of the average turbine in a closely-spaced, spanwise-infinite row compared to a free-standing turbine. The figure shows that blockage can significantly increase the power output of turbines in an infinite row. Our simulation results are in agreement with previous experimental observations by McTavish et al. [98. 113], who showed that flow blockage can increase the turbine power production by $3 \%$ to $9 \%$ for $1 \leqslant s_{y} \leqslant 2$. Here, the main effect is the speed-up of the flow between the closely-spaced turbines. Due to this Venturi effect, the power output of the turbines in the array increases compared to the power output of an isolated turbine. The observed effect is more prominent for the $s_{y}=1.26$ row compared to the less dense $s_{y}=1.57$ case. Furthermore, we observe that the relative power output increases approximately linearly with $C_{T}^{\prime}$.

It is important to consider that the domain height may influence this result. As the turbines are located relatively close to the ground, the wind cannot accelerate under the wind turbines. This means that the air is essentially limited to flow through or over the wind turbine array. However, the height of the ABL may limit the flow acceleration above the wind farm. We selected a domain height $H=1000 \mathrm{~m}$ because it is a typical height for a neutral ABL [18]. To test the potential effect of vertical confinement, we increased the domain height. We find that for $C_{T}^{\prime}=5 / 3, s_{y}=1.26$, the average power output of a turbine in an infinite row compared to that of a free-standing turbine is only $0.9 \%$ lower when the domain height is increased from $H=1000 \mathrm{~m}$ to $2000 \mathrm{~m}$. Regardless, we emphasize that for realistic atmospheric conditions, the capping inversion limits the ABL height from several hundred meters to about $1000 \mathrm{~m}$. 
(a)

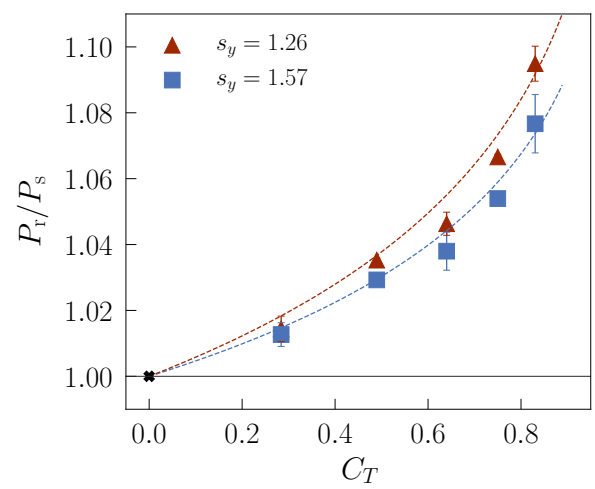

(b)

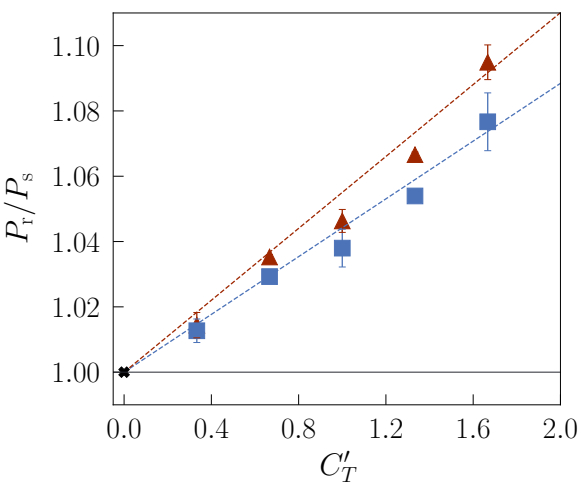

Figure 5.2: The average power output of a turbine in an infinite, closely-spaced row relative to the average power output of a free-standing turbine as a function of a) $C_{T}$ and b) $C_{T}^{\prime}$. The dashed lines indicate the least-squares fit to the $\mathrm{P}_{\mathrm{r}} / \mathrm{P}_{\mathrm{s}}$ versus $C_{T}^{\prime}$ data in (b) and the corresponding lines in (a) are obtained using Equation 5.4

\subsubsection{Blockage effects for a wind farm}

Here, we analyze the performance of the first row of different wind farm layouts to address how placing additional rows downstream affects the performance of the turbines in the first row. We observed that the blockage effect increases linearly with $C_{T}^{\prime}$ for an infinite row when compared to a free-standing turbine. However, placing turbines downstream also introduces a blockage effect. Figure 5.3 shows the average power output of a turbine in the first row of a wind farm compared to the average power output of a turbine within a corresponding solitary row for various thrust coefficients and streamwise spacing. The figure shows that the average power production of the turbines in the first row of each wind farm is lower than the corresponding isolated row and decreases approximately linearly with $C_{T}$. This behavior is due to the presence of the downstream turbines which force more of the flow to go over the wind farm, lowering the velocity at hub-height at the entrance of the wind farm. Therefore, we find that the blockage effect is more pronounced when the streamwise spacing is smaller, i.e., for denser wind farms. Though we compare different layouts, the same linear dependence was also observed by Segalini and Dahlberg; however, we do not observe that this global effect saturates at high $C_{T}$ as they did 90]. Besides, we note that this global blockage effect contrasts the blockage effect observed in the previous section which depends linearly on $C_{T}^{\prime}$, emphasizing that different physical mechanisms play a role. 
(a)

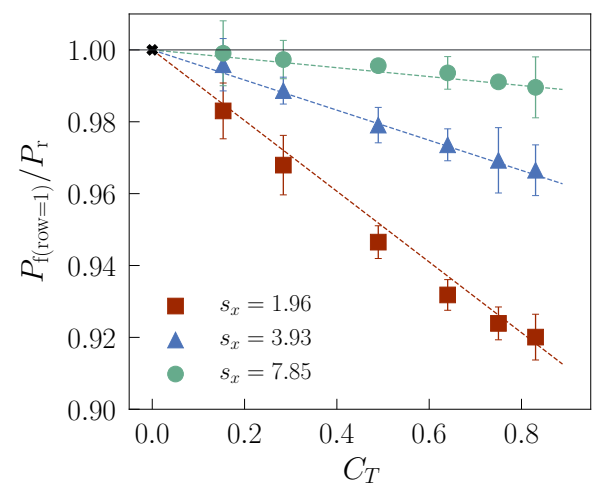

$(b)$

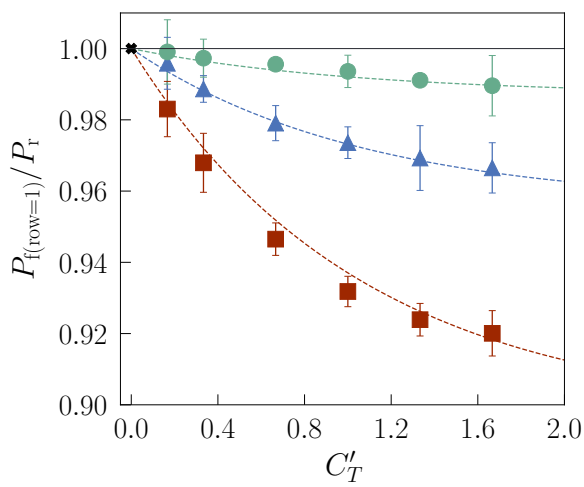

Figure 5.3: The power output of the average turbine in the first row of a wind farm with $s_{y}=1.57$ and different $s_{x}$ is compared to that of a solitary, infinite row as a function of a) $C_{T}$ and b) $C_{T}^{\prime}$. The dashed lines indicate the least-squares fit to the $\mathrm{P}_{\mathrm{f}(\mathrm{row}=1)} / \mathrm{P}_{\mathrm{r}}$ versus $C_{T}$ data in (a) and the corresponding lines in (b) are obtained using Equation 5.4

In Figure 5.4 we compare the relative performance of the average turbine in the first row of a wind farm to a free-standing turbine as a function of the thrust coefficient. Here, we observe the effect of the two types of blockage that we have already established. First, the beneficial effect of close lateral spacing is present, where the average power output of a turbine in an infinite row relative to a free-standing turbine increases approximately linearly with $C_{T}^{\prime}$. Second, the effect of downstream, closely-spaced turbines was shown to reduce the performance of the first row linearly with $C_{T}$. The results in Figure 5.4 suggest that the relative performance of the first row of a wind farm compared to a freestanding turbine depends on the thrust coefficient but without a universal relationship. For $s_{x}=1.96$, the average turbine in the first row produces less power than a freestanding turbine for each thrust coefficient. The layouts with a lower turbine density $\left(s_{x}=3.93,7.85\right)$ exhibit an overall relative benefit from the blockage. This contrasts the results by Bleeg et al. [101], who showed that the performance of the first row decreases compared to an isolated reference turbine. However, we note that we are considering a spanwise-infinite wind farm, which does not allow flow around the wind farm, and this could enhance the observed blockage effects in our case. 
$(a)$

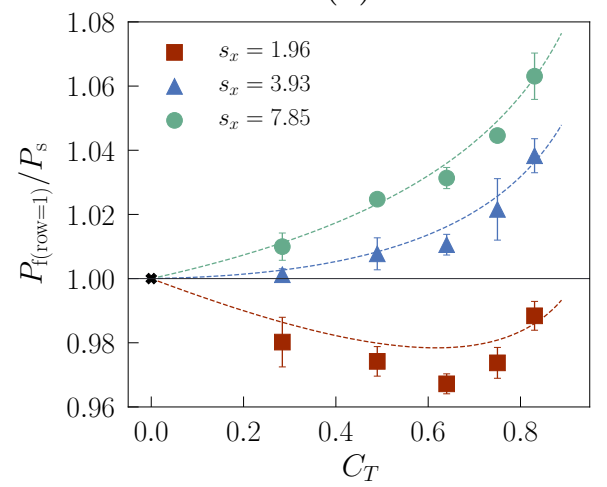

$(b)$

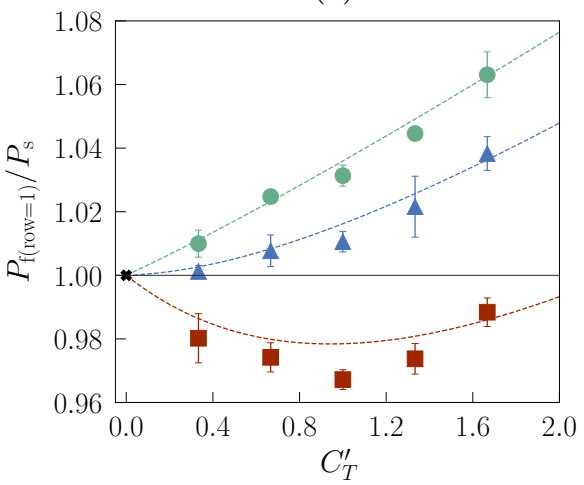

Figure 5.4: The power output of the average turbine in the first row of a wind farm with $s_{y}=1.57$ and different $s_{x}$ is compared to that of a free-standing turbine as a function of a) $C_{T}$ and b) $C_{T}^{\prime}$. The dashed lines are calculated from the fits obtained in Figures 5.2 and 5.3

\subsection{Conclusions}

We used large eddy simulations to study the impact of the turbine thrust coefficient on the blockage effect. We compared the performance of the first row of a tightly packed wind farm, a solitary infinite turbine row, and a free-standing turbine. Overall, we have shown that the blockage effect is enhanced as the thrust coefficient increases. First of all, the average turbine in a closely-spaced infinite row produces more power than a free-standing turbine and the relative power output increases approximately linearly with $C_{T}^{\prime}$. This relative benefit in performance was also observed by others using both models and experiments 98099 . 113 119. We acknowledge that the observed performance enhancement may be more pronounced than in real wind farms because we consider spanwise-infinite arrays in which the flow cannot go around the wind farm [101]. Secondly, we observed that the average turbine in the first row of a wind farm produces less power than a turbine within the corresponding solitary row. In agreement with the results of Segalini and Dahlberg [90], we find that this blockage effect is approximately linear with $C_{T}$. Ultimately, our results show that the power output of a turbine depends on the layout of the wind farm as well as the turbine thrust coefficient. Future work should investigate the effect of the wind farm layout (i.e. different combinations of $s_{x}$ and $s_{y}$ ) and the effect of the lateral confinement on these blockage effects. 


\section{Chapter 6:}

\section{WIND FARM BLOCKAGE IN A STABLE ATMOSPHERIC BOUNDARY LAYER}

A wind farm acts as an obstacle, blocking and deflecting incoming airflow. We show that the turbine power production of the first row of a wind farm can be negatively impacted by the presence of the downstream turbines. Most studies addressing flow blockage have considered neutral atmospheric boundary layer $(A B L)$ conditions; meanwhile, the $A B L$ is often thermally stratified. We investigate the effect of thermal stratification on wind farm blockage using large eddy simulations. Specifically, we systematically increase the surface cooling rate creating a stably stratified $A B L$. Then, we compare the performance of the first row of a wind farm to a solitary row of turbines in order to isolate blockage effects caused by downstream turbines. We observe that the slow-down of the inflow at hub-height, known as induction, is more pronounced preceding a wind farm than a solitary row. Furthermore, this velocity deficit is exacerbated when the downstream turbines are placed more closely or when the atmospheric stability increases. We find that the slow-down and deflection of incoming wind negatively impacts the power output of the first row of a wind farm. Future studies and wind farm planning should consider that blockage is influenced by stable stratification.

To be submitted as: Jessica M. I. Strickland, Srinidhi N. Gadde, and Richard J. A. M. Stevens, Wind farm blockage in a stable atmospheric boundary layer using large eddy simulations, Renewable Energy. 


\subsection{Introduction}

Wind farms are becoming increasingly prevalent as the world scrambles to transition to renewable energy sources. Interested parties aim to quantify and optimize wind farm productivity, demanding accuracy that requires better understanding of the fluid physics involved. A turbine array influences flow both upstream and downstream. The impact of downstream effects, such as turbine wake effects, on the power production are wellestablished [79 121 122]. However, upstream effects, such as flow blockage, are known but relatively unexplored. More insight is imperative for successful incorporation of flow blockage effects into commercial wind farm models and power predictions.

Flow blockage is characterized by the influence that an upcoming obstruction has on the incoming wind. Essentially, turbines influence the inflow, therefore altering the kinetic energy available for extraction. An induced velocity deficit at hub-height precedes even a single turbine as explained in Chapter 4 Section 4.1. The area affected is defined as the induction region and has been demonstrated using numerical models 102 112], and observed in both wind tunnel experiments 92 ] and field measurements [93 94]. When turbines are clustered, the induction is enhanced and the more prominent velocity deficit extends farther upstream. Recently, studies have started exploring the induction zone preceding large turbine arrays. Branlard and Meyer Forsting [102] and Branlard et al. [103] showed that the flow velocity $2.5 \mathrm{D}$ upstream of a wind farm can be reduced by $2 \%$. Both Bleeg et al. [101], and Chapter[4 of this thesis, observed induction up to as far as $10 D$ in front of the first row.

The incoming wind is deflected and this flow blockage impacts turbine productivity. We showed in Chapters 4 and 5 that adding turbines in the spanwise or streamwise direction can influence the flow available neighboring turbines. For instance, close laterally-placed turbines positively influence power production in a neutral $\mathrm{ABL}$, in agreement with wind tunnel experiments [123] and simulations [99]. In this case, deflected flow is forced into the rotor-swept area of a neighboring turbine, increasing the power output. In contrast, close downstream turbines negatively impact upstream turbines as more wind is deflected over the array. Nygaard et al. [91] introduced a wind farm blockage model based on the accumulation of single-turbine induction effects. While they captured the blockage trends observed in field measurement data, they acknowledge that the model underestimates the magnitude of the effect. Wind farm scale blockage was also observed by Segalini et al. 90 105] using wind tunnel experiments with uniform inflow, establishing an empirical relationship that captures the effect of flow blockage on the performance of the first wind farm row. This trend was supported in Chapter 4 using large eddy simulations with turbulent inflow. Even so, these results pertain to a neutral $\mathrm{ABL}$, neglecting thermal stratification, which in reality, is commonly present. 


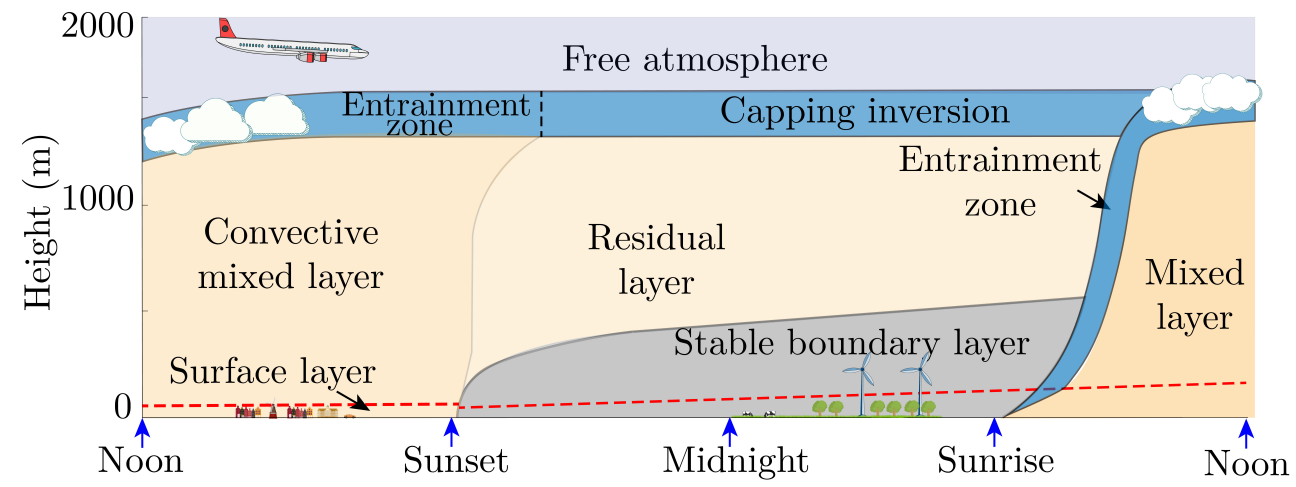

Figure 6.1: Idealized diurnal evolution of the ABL on land consisting of three main layers: a turbulent mixed layer, a less-turbulent residual layer containing former mixed-layer air, and a nocturnal stable boundary layer of sporadic turbulence. Figure recreated by Srinidhi N. Gadde, based on Fig. 1.7 of Stull (1988) [124].

The ABL is the lower region of the atmosphere which is in contact with and influenced by the Earth's surface. In general, thermal stratification of this layer is driven by radiation forcing and adiabatic mixing [124], influencing the velocity profile and determining the depth of the ABL, as shown in Figure 6.1 A neutral ABL forms when there is adiabatic mixing of air driven by a pressure-gradient and cloud cover preventing strong radiation forcing. A stable ABL is characterized by a negative vertical temperature gradient, such that the surface is cooler than the air above it. Therefore, a stable ABL commonly occurs at night or when warm air flows over a cold surface such as polar oceans. In this case, the cooler denser air near the surface is not prone to rise, which means there is less turbulent mixing and the depth of this layer is relatively shallower, typically between $100-500 \mathrm{~m}$ [125]. Generally, mixing in this boundary layer is generated by shear and destroyed by buoyancy and viscosity [83].

Large eddy simulation studies have been adapted to thermally stratified ABLs

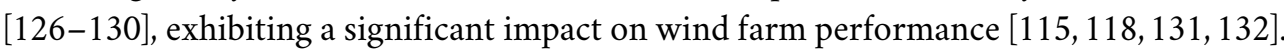
Yet, the effect of stable stratification on wind farm blockage remains relatively unknown. Wu et al. [115] showed that induction is stronger when the atmosphere is stably stratified, and suggest that the cause is related to a feedback of triggered gravity waves. Simley et al. [95] also showed that stability may enhance the induction preceding a single turbine. And recently, Gomez et al. [133]. combined the Weather Research and Forecasting model (WRF) with large eddy simulations to study the blockage effect under two different stability conditions, stating that the blockage effect is enhanced due to limited vertical mixing that restores momentum. 
Thermal stratification of the ABL influences air movement, and thus, the impacts of blockage are not fully represented when only neutral ABL conditions are considered. Therefore, we investigate the influence of thermal stratification on blockage by performing large eddy simulations with a stably stratified $\mathrm{ABL}$ and different wind turbine layouts. We systematically cool the surface, varying the rate in Kelvin per hour $C_{r}[\mathrm{~K} / \mathrm{h}]$, as well as the streamwise turbine spacing $S_{x}[\mathrm{~m}]$. In our simulations, we do not impose a capping inversion, eliminating gravity waves, in order to isolate the effect of surface cooling. First, more generally, we examine the influence of stable ABL conditions on the performance of the different wind turbine layouts. Then, we focus on flow blockage caused by downstream turbines by comparing the first row of a wind farm to that of a solitary row in different stable ABL conditions. Ultimately, our objective is to determine the impact of thermal stratification and downstream turbine spacing on the induction region and the power output at the first row of the wind farm. The remainder of this chapter is organized as follows. The numerical methods and simulation case specifications are detailed in Section 6.2. the results are presented and discussed in 6.3 and a summary of the findings is provided in Section 6.4

\subsection{Numerical framework}

\subsubsection{Large eddy simulations with thermal stratification}

We use large eddy simulations to investigate wind farm blockage in a stably stratified ABL. In the previous chapters, we considered a neutral ABL, so here we describe the modifications required to incorporate thermal stratification. The large eddy simulation code is based on the version developed by Albertson and Parlange [63 64] and has been further updated to successfully simulate wind farms in stably stratified ABLs $125,134,135$. The governing equations are as follows. We integrate the filtered Navier-Stokes equations with buoyancy modeled using the Buossinesq approximation:

$$
\begin{aligned}
\partial_{t} \tilde{u}_{i}+\partial_{j}\left(\widetilde{u}_{i} \widetilde{u}_{j}\right)= & -\partial_{i} \tilde{p}-\partial_{j} \tau_{i j}+g \beta\left(\widetilde{\theta}-\tilde{\theta}_{0}\right) \delta_{i 3} \\
& +f_{c}\left(U_{g}-\widetilde{u}\right) \delta_{i 2}-f_{c}\left(V_{g}-\widetilde{v}\right) \delta_{i 1}+\tilde{f}_{x} \delta_{i 1}+\tilde{f}_{y} \delta_{i 2}, \\
\partial_{i} \widetilde{u}_{i}= & 0, \\
\partial_{t} \tilde{\theta}+\tilde{u}_{j} \partial_{j} \tilde{\theta}= & -\partial_{j} q_{j},
\end{aligned}
$$

where tilde $\left({ }^{\sim}\right)$ represents spatial filtering at the resolved scale $\tilde{\Delta}$, and thus $\widetilde{u}_{i}=(\widetilde{u}, \widetilde{v}, \widetilde{w})$ is the grid-scale velocity. On the right side of Equation $6.1 \tilde{p}=\tilde{p}^{*} / \rho+\sigma_{k k} / 3$ represents the modified pressure by adding the trace of sub-filter stress $\sigma_{k k} / 3$ to the kinematic pressure $\tilde{p}^{*} / \rho$, and $\rho$ represents the density of air. The buoyancy term contains the 
grid-scale potential temperature $\widetilde{\theta}$, the acceleration due to gravity $g$, and the buoyancy parameter $\beta=1 / \theta_{0}$, where $\theta_{0}$ is the reference potential temperature, and $\delta_{i j}$ is the Kronecker delta function. The Coriolis forcing is expressed in two components where the Coriolis parameter $f_{c}=2 \Omega \sin \phi=1.159 \times 10^{-4} \mathrm{rad} / \mathrm{s}, \Omega$ is the rotation of the Earth, and $\phi=52.8^{\circ}$, corresponding to the Dutch North Sea. The flow is driven by a mean pressure gradient $p_{\infty}$, represented by the geostrophic wind where $U_{g}=-\frac{1}{\rho f_{c}} \frac{\partial p_{\infty}}{\partial y}$ and $V_{g}=\frac{1}{\rho f_{c}} \frac{\partial p_{\infty}}{\partial x}$ are the streamwise and spanwise components, respectively, and the magnitude is $G=12 \mathrm{~m} / \mathrm{s}$. The turbine forces $\widetilde{f}_{i}$ are modeled with the filtered actuator disk approach as described in Chapter 3 [ $\left.\begin{array}{l|l|l|l|l}18 & 77 & 136 & 137\end{array}\right]$. Remaining are $\tau_{i j}=\widetilde{u_{i} u_{j}}-$ $\widetilde{u}_{i} \widetilde{u}_{j}$ and $q_{j}=\widetilde{u_{j} \theta}-\widetilde{u}_{j} \widetilde{\theta}$, which represent the traceless part of the sub-grid scale stress tensor and the sub-grid scale heat flux tensor, respectively. They are modeled as

$$
\begin{aligned}
\tau_{i j} & =\widetilde{u_{i} u_{j}}-\widetilde{u}_{i} \widetilde{u}_{j}=-2 \nu_{T} \widetilde{S}_{i j}=-2\left(C_{\mathrm{smag}} \Delta\right)^{2}|\widetilde{S}| \widetilde{S}_{i j}, \\
q_{j} & =\widetilde{u_{j} \theta}-\widetilde{u}_{j} \tilde{\theta}=-\nu_{\theta} \partial_{j} \widetilde{\theta}=-\left(D_{\mathrm{smag}} \Delta\right)^{2}|\widetilde{S}| \partial_{j} \tilde{\theta},
\end{aligned}
$$

where $\widetilde{S}_{i j}=\frac{1}{2}\left(\partial_{j} \widetilde{u}_{i}+\partial_{i} \widetilde{u}_{j}\right)$ represents the resolved strain rate tensor, $\nu_{T}$ represents the turbulent eddy viscosity, $C_{\text {smag }}$ represents the Smagorinsky coefficient for the subgrid scale stresses, $\nu_{\theta}$ is the eddy heat diffusivity, $D_{\text {smag }}$ represents the Smagorinsky coefficient for the sub-grid scale heat flux, and $|\widetilde{S}|=\left(2 \widetilde{S}_{i j} \widetilde{S}_{i j}\right)^{1 / 2}$. We use a tuning-free, scale-dependent anisotropic minimum dissipation model [125, $138-140]$ to calculate the Smagorinsky coefficient dynamically, which makes the model particularly suitable for inhomogeneous flows, such as the flow through a wind farm.

The governing equations are solved as follows. We use a pseudo-spectral method to calculate the partial derivatives in the streamwise and spanwise directions. The vertical direction is treated with a second-order central difference method. The solution is advanced in time by a second-order accurate Adams-Bashforth scheme. The aliasing errors resulting from the folding back of high wave number energy to the resolved scales due to the calculation of non-linear terms in physical space are prevented by using a $3 / 2$ anti-aliasing method [141]. For point-wise energy conservation, the convective term in the equation 6.1 is written in the rotational form [142]. More information about the numerical method can be found in reference [63].

The computational domain is discretized uniformly in the horizontal direction, but a stretched grid is applied in the vertical direction. Specifically, the grid is stretched above $z=1000 \mathrm{~m}$ in order to reduce the resolution and thus computational costs well above the stable boundary layer height. In addition to stretching, the computational grid is staggered in the vertical direction with the first grid point for $\widetilde{u}, \widetilde{v}$, and $\widetilde{\theta}$ located at a distance $\Delta_{z} / 2$ above the ground while the computational plane for the vertical velocity $\widetilde{w}$ is located at the ground. No-slip and free-slip boundary conditions with zero vertical 
velocity $(\widetilde{w}=0)$ are used at the top and bottom boundaries, respectively. In wall-modeled large eddy simulations of $\mathrm{ABL}$, the first grid point generally lies above the surface, within the surface layer, and the Monin-Obukhov similarity theory [68 143$]$ can be used to model the instantaneous shear stress $\tau_{i 3 \mid w}$ and buoyancy flux $q_{*}$ at the wall as follows:

$$
\begin{gathered}
\tau_{i 3 \mid w}=-u_{*}^{2} \frac{\widetilde{u}_{i}}{\widetilde{u}_{r}}=-\left(\frac{\widetilde{u}_{r} \kappa}{\ln \left(z / z_{o}\right)-\psi_{M}}\right)^{2} \frac{\widetilde{u}_{i}}{\widetilde{u}_{r}}, \\
q_{*}=\frac{u_{*} \kappa\left(\theta_{s}-\widetilde{\theta}\right)}{\ln \left(z / z_{o}\right)-\psi_{H}},
\end{gathered}
$$

where $u_{*}$ is the frictional velocity, $z_{0}$ is the roughness length, $\kappa$ is the von Kármán constant, $\widetilde{u}_{r}=\sqrt{\widetilde{u}^{2}+\widetilde{v}^{2}}$ is the filtered velocity magnitude at the first grid level, and $\theta_{s}$ is the filtered potential temperature at the surface, which is reduced at a set cooling rate of $C_{r}$ in Kelvin per hour $[\mathrm{K} / \mathrm{h}]$. When $C_{r}=0 \mathrm{~K} / \mathrm{h}$, a conventionally neutral ABL is generated which has been confirmed by Gadde et al. [125], asserting that in this case, the surface heat flux $q_{*}$ is negligible. This method of establishing stable stratification by reducing the surface temperature at a constant rate versus being driven by a constant heat flux also yielded similar results for Kumar et al. [144]. Therefore, for the purpose of this study we implemented the former. In our LES, we do not impose a capping inversion because this is known to trigger gravity waves which would ultimately affect the flow within the domain $\left[\begin{array}{l|l|l|l|l|l|l|l|l|}30 & 115 & 118 & 145 & 146\end{array}\right]$ and prevent us from isolating the blockage effects we aim to study. For stable ABLs, we use the stability corrections for momentum and heat flux suggested by reference [147], i.e. $\psi_{M}=-4.8 z / L$ and $\psi_{H}=-7.8 z / L$, respectively, where $L=-\left(u_{*}^{3} \theta_{0}\right) /\left(\kappa g q_{*}\right)$ is the surface Obukhov length. The wallmodel is implemented as explained in Bou-Zeid et al. [67] and the wall-layer fluxes and stresses are calculated using the filtered velocities at the first grid point above the ground.

\subsubsection{Simulation specifications}

We investigate the impact of the streamwise turbine spacing $S_{x}$ and the surface cooling rate $C_{r}$ on flow blockage by performing 20 large eddy simulations (summarized in Table 6.1. We compare a wind farm with eight rows $\left(N_{T, x}=8\right)$ to a solitary infinite row of turbines (illustrated in Figure 6.2). The number of turbines per row $\left(N_{T, Y}=8\right)$ and the dimensionless spanwise spacing $\left(s_{y} / D=4.608\right)$ remain constant in order to isolate changes in blockage caused by downstream turbines and not by spanwise neighboring turbines. Due to the spanwise periodic boundary conditions, each row is infinite which may enhance the blockage effect because air is prevented from flowing around the wind farm. However, the results of Chapter 4 Section 4.3.3 determined that the streamwise 
blockage trends are not influenced by the spanwise wind farm extent. We also determined that blockage is most pronounced when the turbine spacing is small and with that in mind, we vary the dimensionless turbine streamwise spacing $\left(S_{x} / D=7,5,3.5,1.75\right)$ while all other wind farm parameters remain constant. The turbine diameter is $D=125 \mathrm{~m}$, the hub height is $z_{h}=100 \mathrm{~m}$, and the modified disk-based thrust coefficient is $C_{T}^{\prime}=5 / 3$.

The turbine arrays are placed within a computational domain with dimension lengths $L_{x} \times L_{y} \times L_{z}=17.28 \mathrm{~km} \times 4.608 \mathrm{~km} \times 8.6 \mathrm{~km}$, discretized by the number of grid points $N_{x} \times N_{y} \times N_{z}=1440 \times 384 \times 384$. Therefore, the corresponding horizontal resolution is $\Delta_{x}=\Delta_{y}=12 \mathrm{~m}$. The vertical resolution when $z<1000 \mathrm{~m}$, and therefore across the turbine disk, is $\Delta_{z}=5 \mathrm{~m}$. The (first) turbine row is placed at $x=6 \mathrm{~km}$ in each simulation which is more than sufficient to properly capture the induction region 92 95-97.

The simulations are carried out in two stages. In the initial stage, a stable ABL domain develops without turbines and once a quasi-steady state is achieved (approximately 7 hours flow time), the information from this domain is used for the second stage. The second stage applies the concurrent precursor method [32]: data from the stable ABL simulation is sampled and used as an inflow condition for a second domain in which the turbines are placed. The simulations are performed long enough to ensure that the power production has reached a quasi-stationary state. Then, we collect time-averaged statistics between $8-9$ hours. For our study, the initial stage is implemented with four different surface cooling rates $\left(C_{r}=0,0.25,0.5,1.0 \mathrm{~K} / \mathrm{h}\right)$ and then in the second stage, one of the five different turbine layouts is introduced.

(a) Solitary row

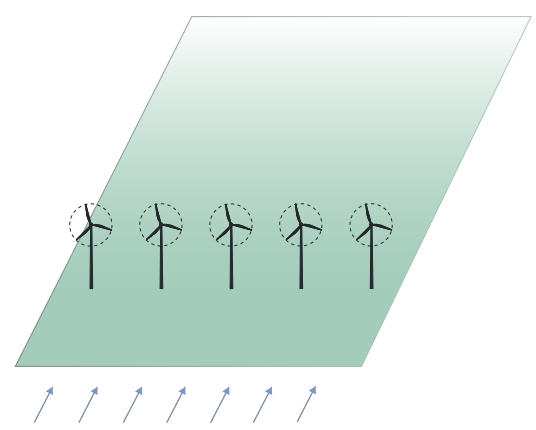

(b) Wind farm

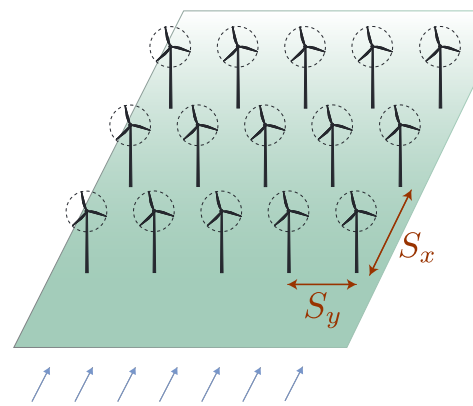

Figure 6.2: Schematic depicting the two wind turbine layouts: (a) solitary infinite row and (b) wind farm with eight rows. The inflow direction is indicated by the arrows and the spanwise and streamwise turbine spacing are $S_{y}$ and $S_{x}$, respectively. 


\begin{tabular}{llll}
\hline Case & $N_{T, x}$ & $S_{x}[\mathrm{~m}]$ & $C_{r}[\mathrm{~K} / \mathrm{h}]$ \\
\hline Solitary row & 1 & - & $0.0,0.25,0.5,1.0$ \\
Wind farm & 8 & $7 D$ & $0.0,0.25,0.5,1.0$ \\
Wind farm & 8 & $5 D$ & $0.0,0.25,0.5,1.0$ \\
Wind farm & 8 & $3.5 D$ & $0.0,0.25,0.5,1.0$ \\
Wind farm & 8 & $1.75 D$ & $0.0,0.25,0.5,1.0$ \\
\hline
\end{tabular}

Table 6.1: Summary of wind turbine array simulations with $N_{T, x}$ rows in a stable ABL. The variables of interest are the streamwise spacing $S_{x}$ in meters and the surface cooling rate $C_{r}$ in Kelvin per hour.

\subsection{Results and discussion}

We present the simulation results, beginning with an overview of the wind farm performance in a stably stratified ABL. Then, we examine blockage effects in the first row of these wind farms, focusing on the influence of the streamwise turbine spacing and atmospheric stability.

\subsubsection{Wind farm performance in a stable $\mathrm{ABL}$}

Our key parameter for exploring thermal stratification is the surface cooling rate $C_{r}[\mathrm{~K} / \mathrm{h}]$. Increasing the cooling rate creates a more stable ABL which can be observed in the potential temperature profiles of the inflow compared in Figure 6.3 a). Horizontal-averaging is denoted by triangle brackets $(\langle\rangle)$ and time-averaging is denoted by a bar $\left(^{-}\right)$. As expected, the potential temperature of the stable ABL is lowest near the surface due to the cooling and increases with height. This negative surface buoyancy affects the turbulence of the ABL. Figure 6.3 (b) shows the corresponding profile of the turbulence intensity defined as $I=\sigma / v_{\text {mag }}$, where $\sigma=\overline{u^{\prime 2}}+\overline{v^{\prime 2}}$, and $v_{\text {mag }}=\sqrt{\bar{u}^{2}+\bar{v}^{2}}$ is the wind magnitude. The turbulence intensity is highest near the surface and, for a given height, the turbulence intensity decreases as the atmosphere becomes more stably stratified. The amount of turbulence will affect wind farm performance as the kinetic energy extracted by the turbines is replenished through turbulent mixing.

The wind profile is also influenced by thermal stratification. Figure 6.3 (c) shows the magnitude of the time- and horizontally-averaged velocity profile for the inflow domain with varied stability. There is a clear distinction between the neutral ABL and the stable ABLs. In particular, we observe a wind velocity maximum for the stable cases which is known as a low-level jet [148]. As the ABL becomes more stable, the velocity maximum 
(a)

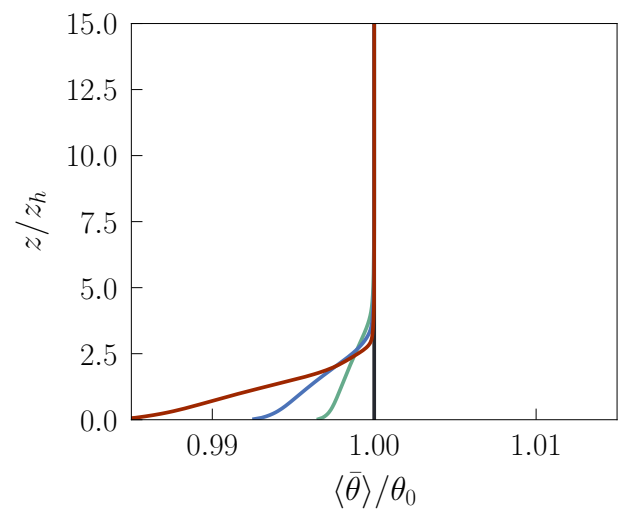

(c)

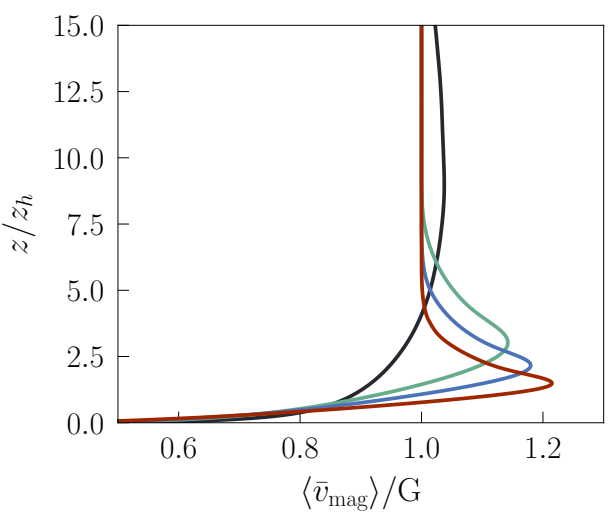

(b)

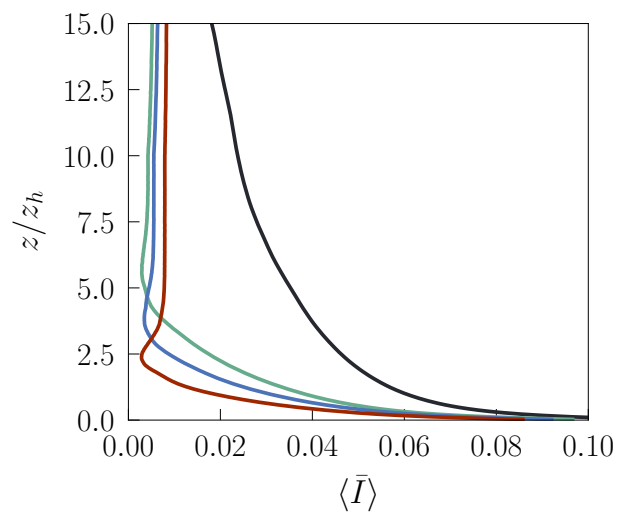

Figure 6.3: The time- and horizontally-averaged (a) potential temperature, (b) turbulence intensity and, (c) wind velocity magnitude profiles as a function of height for the ABL without turbines with varied stability as indicated by the cooling rate $C_{r}$.

of the low-level jet increases while the height at which this occurs decreases. For instance, when $C_{r}=1.0 \mathrm{~K} / \mathrm{h}$, the low-level jet would reside partially within the rotor-swept area of the turbines. Depending on the jet's position and strength, its high energy potential and shear play an important role in sustaining turbulence within the boundary layer, consequently impacting wind farm performance 125,149 .

The influence of thermal stratification on wind farm-ABL interactions is evident when examining wake development. Figure 6.4 shows the time-averaged wind velocity 


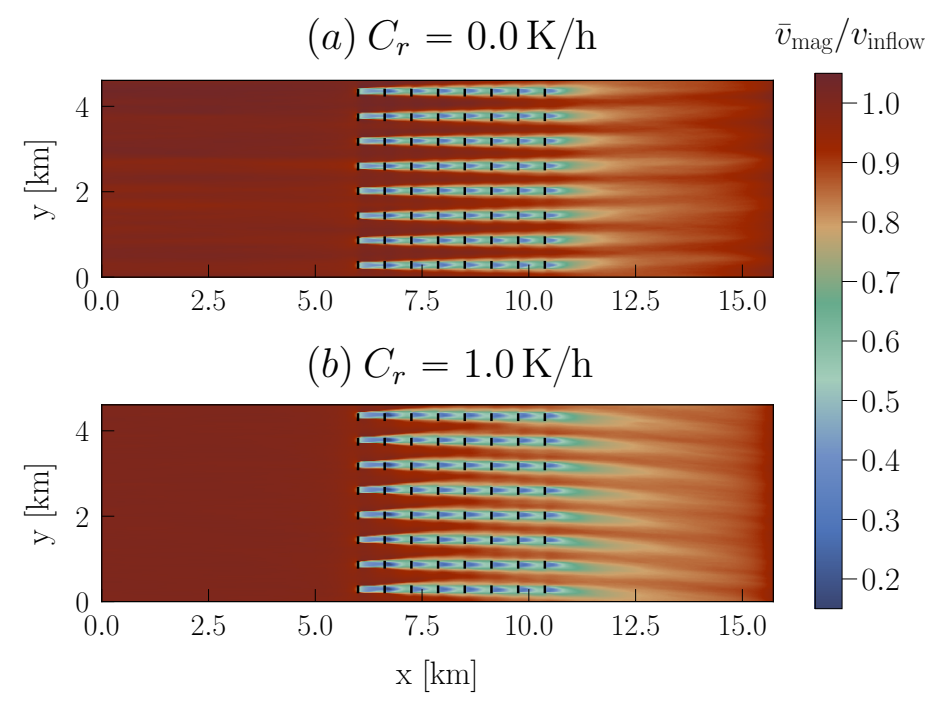

Figure 6.4: The normalized time-averaged wind velocity magnitude for a wind farm $\left(S_{x}=5 D\right)$ at hub-height in (a) neutral and (b) stable ABL conditions.

magnitude at hub-height for the same wind farm layout $\left(S_{x}=5 D\right)$ in both a neutral $\left(C_{r}=0.0 \mathrm{~K} / \mathrm{h}\right)$ and a stable $\left(C_{r}=1.0 \mathrm{~K} / \mathrm{h}\right) \mathrm{ABL}$ conditions. The velocity is normalized by the inflow velocity $v_{\text {inflow }}$, which is the time- and horizontally-averaged value at hub-height in the corresponding inflow domain without turbines. Comparing both figures, the stable case receives less variable inflow due to the hindered turbulent motions. Effects of the high-velocity streaks observed in the neutral case are minimized using a shifting technique [69]. In both cases, clear velocity deficits are visible behind each turbine as momentum is extracted from the wind. The shape and magnitude of this wake is dependent on the stability. Specifically, we observe that the veering of the wake due to the Coriolis force is stronger in Figure 6.4 b) because of the relatively high stability and stronger shear. Furthermore, in the stable case, the wakes progress farther downstream before recovering due to the reduced turbulent mixing and therefore less entrainment of higher velocity air from above the wind farm [150].

Both thermal stratification and the turbine layout will affect the amount of power generated by the wind farm. Figure 6.5 presents the average power output of each turbine row within a wind farm normalized by the average power of the first row of that wind farm for each of the simulations. As expected, the downstream rows do not perform as well as the first row due to wake effects which are stronger in more stable conditions. 
The relative power production is strongly dependent the streamwise spacing because sufficient distance is required for a turbine wake to recover. For instance, a wind farm where $S_{x}=1.75 D$ is inadvisable as the downstream rows produce less than $25 \%$ of the power of the first row. Beyond the second and third row, an asymptote is reached and the relative performance of each downstream row is similar because of a balance between extracted kinetic energy which is partially replenished through entrainment as the flow progresses downstream. We observe this behavior for most cases in Figure 6.5 However, deviation from this trend is apparent for the most stable case $\left(C_{r}=1.0 \mathrm{~K} / \mathrm{h}\right)$, especially when the streamwise spacing is larger. Gadde et al. [125]151] related the higher relative power of the second to fourth row to higher entrainment from the low-level jet which is lower when the $\mathrm{ABL}$ is more stable (refer to Figure 6.3( $\mathrm{c}$ )). However, the veering observed in Figure 6.4 can cause asymmetries in wake exposure between the rows at the entrance and the end of the wind farm. The veering effects are less influential when the streamwise turbine spacing is small. Nonetheless, the conclusions made here, normalizing by the

(a) $S_{x}=7 D$

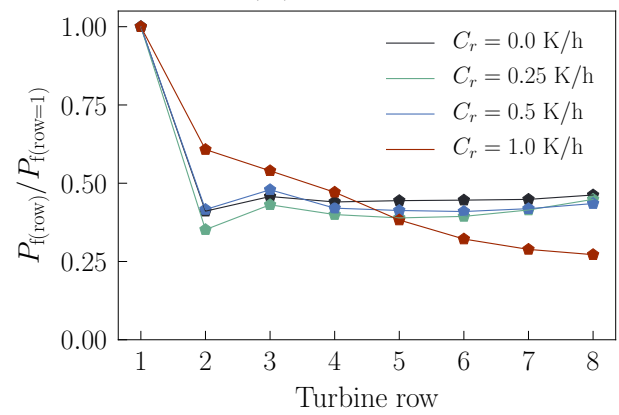

(c) $S_{x}=3.5 D$

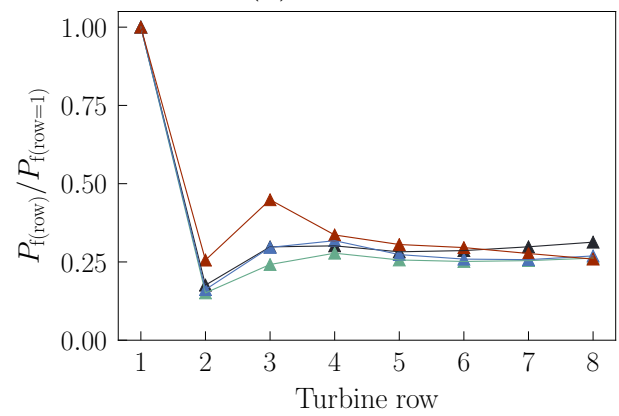

(b) $S_{x}=5 D$

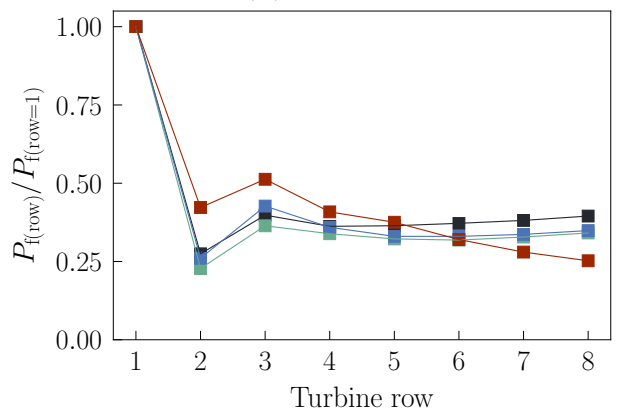

(d) $S_{x}=1.75 D$

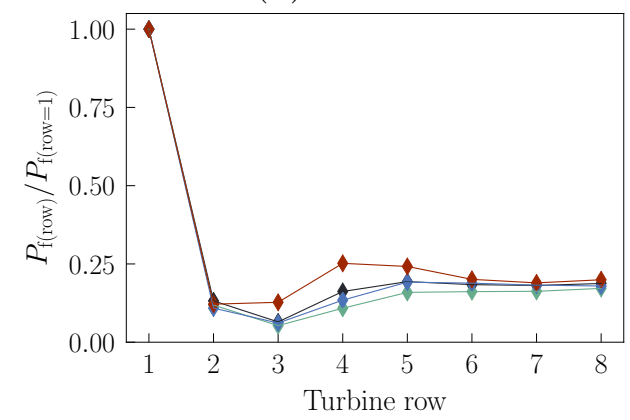

Figure 6.5: The average power production of each row of a wind farm normalized by the first row with different streamwise turbine spacing $S_{x}$ and cooling rate $C_{r}$. 
first row of turbines, only address downstream factors such as the balance between wake losses and recovery. Upstream effects, such as flow blockage, are unaccounted for and require a different reference.

\subsubsection{Wind farm blockage in a stable $\mathrm{ABL}$}

The first row of a wind farm is typically assumed to receive undisturbed flow; however, this is not the case due to wind farm blockage. In Chapters 4 and 5 we established that the first row of a wind farm produces less power when succeeded by close downstream turbines in a neutral ABL. In order to isolate and quantify streamwise blockage in a stable $\mathrm{ABL}$, we compare the first row of a wind farm to a solitary row, while systematically varying the streamwise turbine spacing and surface cooling rate. Figure 6.6 presents the average power of the first row, normalized by the average power of the solitary row, as a function of cooling rate and streamwise turbine spacing. For each wind farm, we observe that the power output of the first row is less than that of a solitary row due to blockage. The wind farm acts as an obstacle and the flow is deflected over the wind farm as opposed to passing through it. For instance, when $S_{x}=1.75 D$ and $C_{r}=1.0 \mathrm{~K} / \mathrm{h}$, the power output of the first row is reduced by approximately $14 \%$, compared to the case without downstream turbines.

(a)

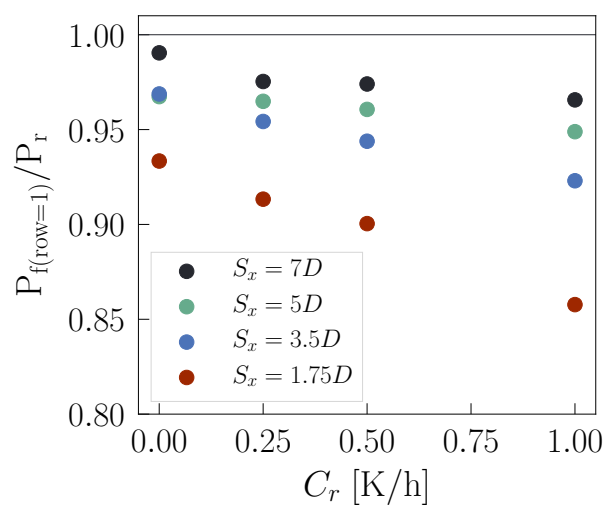

(b)

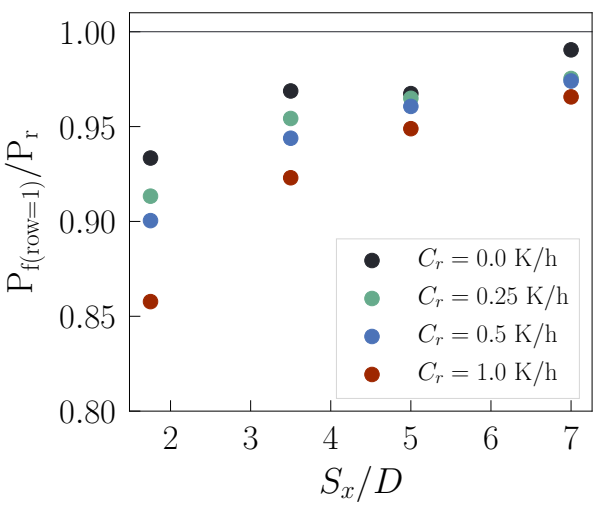

Figure 6.6: The average power production of a turbine in the first row of a wind farm compared to the power production of a corresponding solitary row as a function of (a) the cooling rate and (b) the streamwise turbine spacing. 
The degree to which downstream turbines hinder the performance of the first row is clearly dependent on the streamwise spacing and thermal stability. Figure 6.6 a) shows that for each wind farm layout, the relative performance of the first row decreases as the cooling rate increases. Therefore, as the ABL becomes more stable, the blockage effect is exacerbated. Figure 6.6 (b) shows the blockage effect is also stronger when the streamwise spacing decreases, as observed in Chapters 4 and 5 and other recent studies [90, 102]. Furthermore, as the streamwise spacing increases, blockage appears to be less influenced by the atmospheric stability due to the small magnitude of the blockage effect $(1-5 \%)$ when turbines are placed sparsely downstream.

In order to better understand how flow blockage influences wind farm productivity, we analyze how the wind is affected at the entrance region. Figure 6.7 shows the timeand spanwise-averaged vertical velocity at hub-height for the solitary row and two wind farm layouts with neutral and stable conditions. The vertical velocity is normalized by the wind velocity magnitude of the inflow domain at hub-height. In each figure, we observe an increasing positive vertical velocity in the region preceding the turbine array as air approaches the obstacle and is displaced upwards. Furthermore, the vertical motion of air increases when downstream turbines are introduced and when placed closely. Comparing the two figures, we also observe that the vertical velocity at hub-height is higher in stable conditions for the same turbine layouts. Moreover, the impact of changing the streamwise spacing is more pronounced in a stable ABL. Therefore, the amount of flow deflection depends on the layout of turbines and the stability of the atmosphere.

The induction preceding the turbine arrays is also dependent on the turbine density
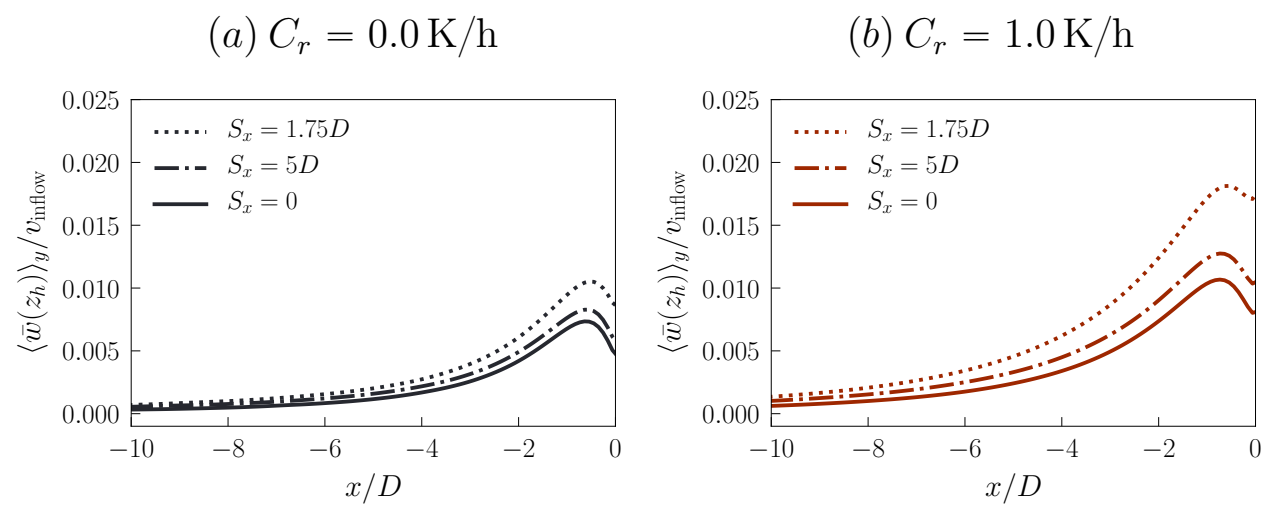

Figure 6.7: The normalized time- and spanwise-averaged vertical velocity for a solitary row $\left(S_{x}=0\right)$ and two wind farms $\left(S_{x}=1.75 D, 5 D\right)$ at hub-height as a function of the position upstream where $x / D=0$ is the location of the first row of turbines for (a) neutral and (b) stable ABL conditions. 
and stability. Figures 6.8 and 6.9 compare the average centerline velocity at hub-height normalized by the inflow centerline velocity at hub-height as a function of the position upstream for different wind farm layouts and stability conditions. The induction of a single turbine, derived from actuator disk theory (refer to Equation 4.1), is plotted as a general reference. In each figure, we observe a clear velocity deficit preceding the turbine arrays where the wind is slowed for several turbine diameters upstream.

In Figures $6.8 \mathrm{a}$ a) and (b), we focus on the effect of the streamwise turbine spacing in neutral and stable conditions, respectively. Specifically, the induction of the solitary row $\left(S_{x}=0\right)$ is compared to two wind farm layouts with different streamwise spacing $\left(S_{x}=1.75 D, 5 D\right)$. In each figure, we observe similar trends as established in Chapter 4 Compared to a single turbine, the velocity deficit preceding a solitary row is stronger and extends farther upstream. Furthermore, the induction preceding an entire wind farm is even stronger and more extensive as the streamwise spacing decreases. Comparing Figures $6.8(\mathrm{a})$ and (b), the induction appears to be more pronounced for the stable case, with more distinction between the different turbine layouts. In fact, for the densest wind farm layout $\left(S_{x}=1.75 D\right)$, the induction region, where the velocity is less than $99 \%$ of the inflow, extends up to $-7.3 D$ and $-9.8 D$ for the $C_{r}=0 \mathrm{~K} / \mathrm{h}$ and $C_{r}=1.0 \mathrm{~K} / \mathrm{h}$ conditions, respectively.

In Figure 6.9 we focus on how stability influences the induction by comparing the cooling rates under consideration for a solitary row and two wind farms where $S_{x}=5 D, 1.75 D$. In each figure, we can clearly see the influence of stability as the velocity deficit becomes more pronounced and extends farther upstream as the cooling rate increases. However, the cooling rate is less influential for the solitary row case (Figure 6.9 a)) and is most influential for the densest wind farm layout (Figure 6.9(c)). The trends we observe for this streamwise slow-down and deflection of wind preceding the turbine arrays, are in good agreement with the blockage effect observed in Figure $6.6 \mathrm{Wu}$ et al. [115] also observe enhanced induction with stability. However, they attribute the dramatic change to gravity waves, as do Allaerts and Meyers [118]. There are no gravity waves in our simulations because we do not impose a capping inversion, suggesting that the enhanced induction when stability increases is related to other physical phenomenon which we will explore further. 
(a) $C_{r}=0.0 \mathrm{~K} / \mathrm{h}$

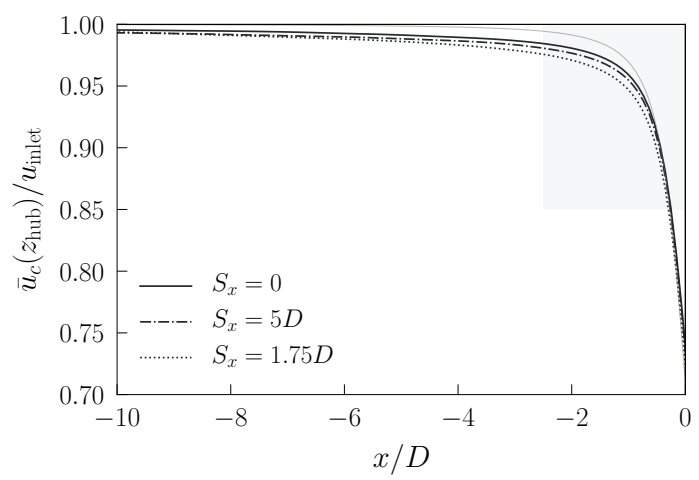

(b) $C_{r}=1.0 \mathrm{~K} / \mathrm{h}$

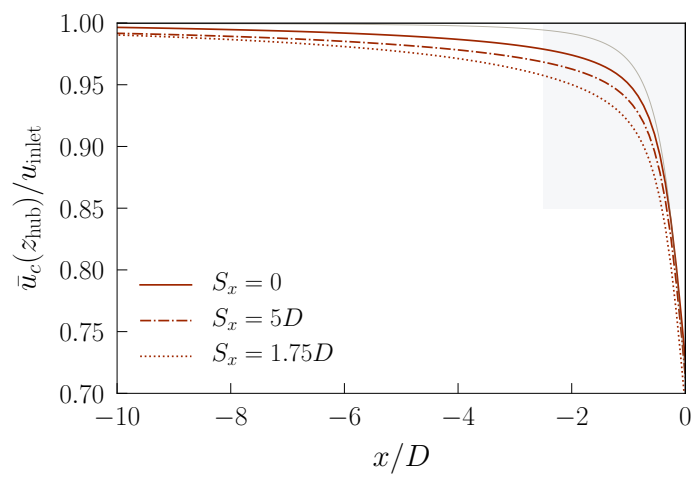

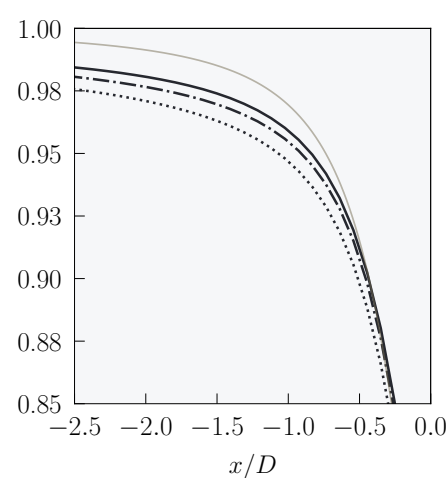

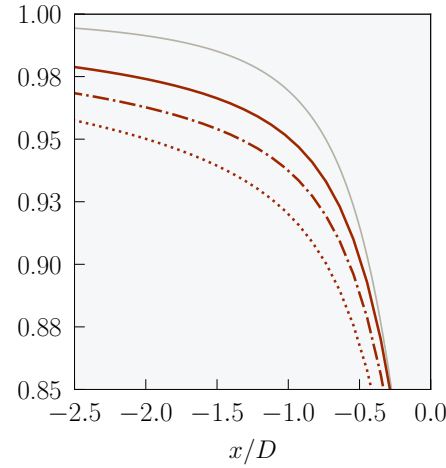

Figure 6.8: The time-averaged streamwise velocity at hub-height along the centerline preceding each turbine normalized by the incoming centerline velocity at hub-height as a function of the position upstream where $x / D=0$ is the location of the (first) row of turbines shown for a solitary row $\left(S_{x}=0\right)$ and two wind farms $\left(S_{x}=1.75 D, 5 D\right)$ in (a) neutral and (b) stable conditions. The brown line is the reference for a stand-alone turbine defined in Equation 4.1 
(a) $S_{x}=0$
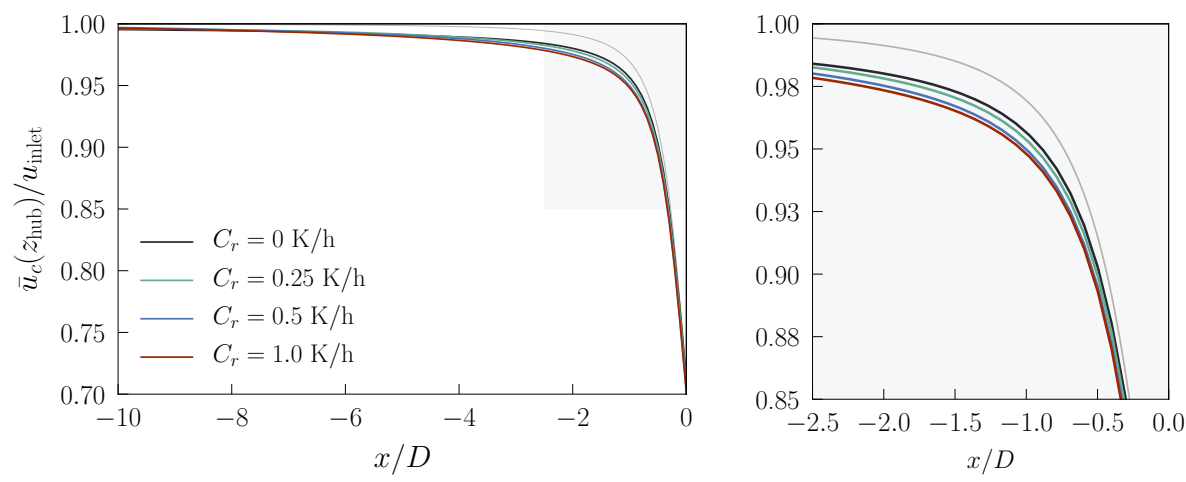

(b) $S_{x}=5 D$
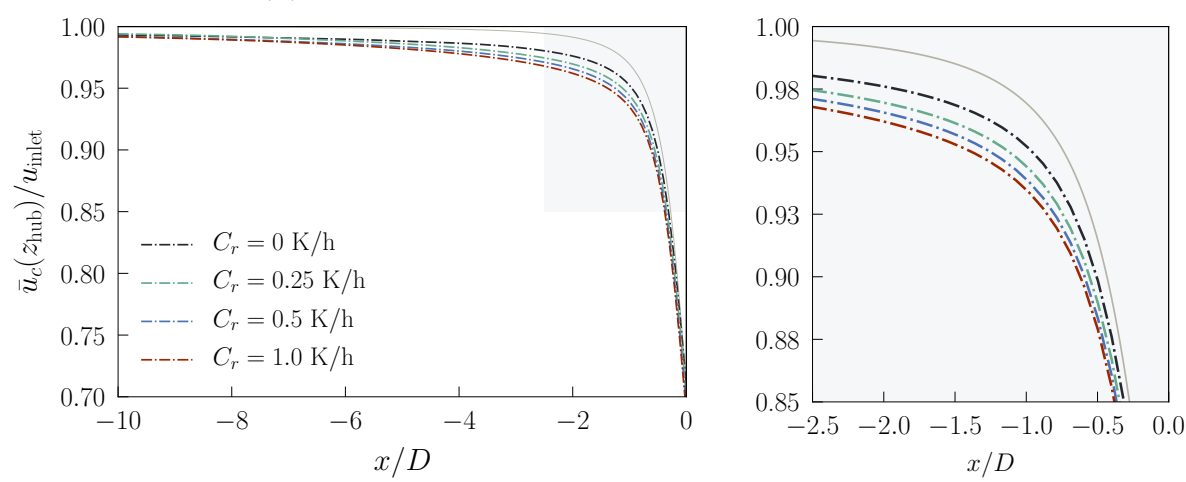

(c) $S_{x}=1.75 D$
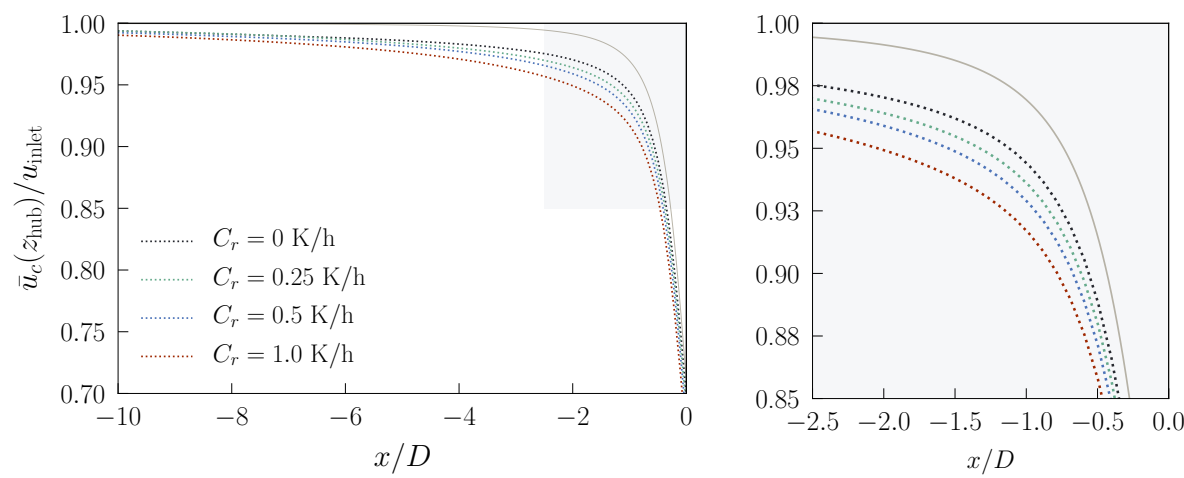

Figure 6.9: The time-averaged velocity at hub-height along the centerline preceding each turbine normalized by the incoming value as a function of the position upstream shown for a solitary row $\left(S_{x}=0\right)$ and two wind farms where $S_{x}=5 D, 1.75 D$. The brown line is the reference for a stand-alone turbine defined in Equation 4.1 

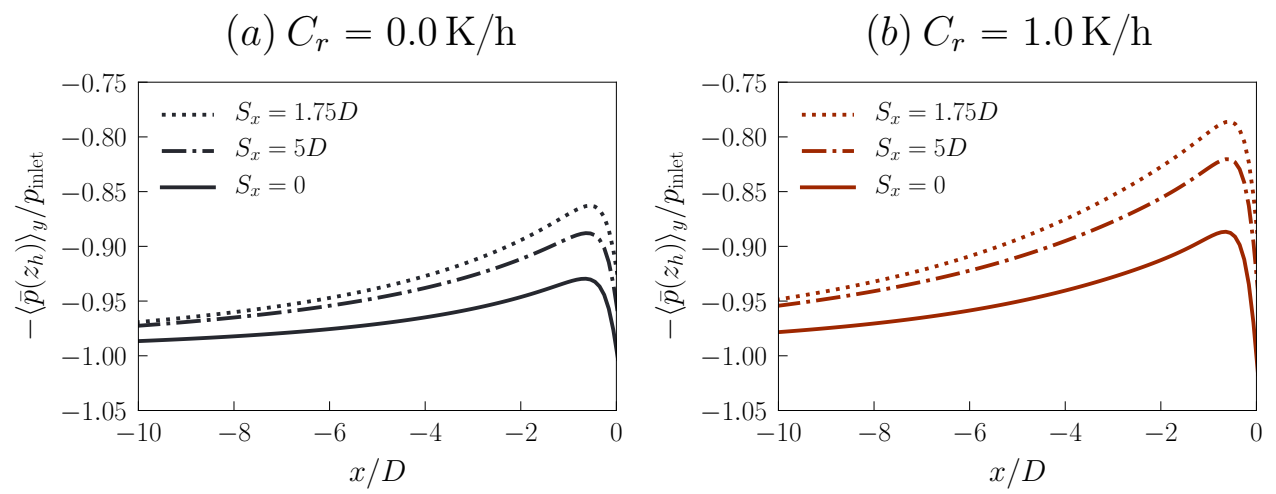

Figure 6.10: The time- and spanwise-averaged streamwise pressure at hub-height normalized by the inlet value at hub-height.

To better understand the behavior of the incoming wind, we examine the horizontal pressure preceding the wind turbine arrays. Pressure is expected to increase approaching the turbine disks as illustrated in Figure 3.3. Chapter 3 . Figure 6.10 shows the time- and spanwise-averaged streamwise pressure at hub-height normalized by the inlet value at hub-height for different wind farm layouts in varied stability conditions. The pressure perturbation is shown as a function of distance upstream and we focus on the induction zone. In Figure 6.10 we observe the adverse pressure gradients preceding the turbine arrays causing the wind slow-down, inherent of the induction. Furthermore, in both figures, adjusting the spacing of downstream turbines influences this pressure change, even when the atmosphere is neutral. We observe that the impact of changing the streamwise turbine spacing is more pronounced in stable conditions. Moreover, when the surface cooling rate increases, the adverse pressure gradient is more prominent as the atmosphere becomes more stable. These trends are reflected in the enhanced induction that we observed.

Altogether, we justify the power reduction caused by blockage effects as follows. Clustering the turbines together creates a high-pressure region and the enhanced adverse pressure gradient slows the wind which is deflected over the wind farm. Placing the downstream turbines closer exacerbates this effect, reducing the power output at the entrance of the wind farm. This effect also increases when the atmosphere becomes more stable. We postulate that the blockage effect from clustering the wind turbines forces a cool parcel of air aloft and a local high-pressure anomaly is created below due to the hydrostatic law [30] and therefore, increasing the stability will enhance the adverse pressure gradient and the induction accordingly. In summary, placing turbines close together creates blockage which can be enhanced by stable stratification, ultimately reducing the expected power output at the first row of a wind farm. 


\subsection{Conclusions}

The purpose of this study was to use large eddy simulations to investigate how blockage is influenced by stable stratification of the atmosphere. First, we examined how the different wind farm layouts performed in stable atmospheric conditions. By systematically adjusting the surface cooling rate, we confirmed that the performance throughout the wind farm is affected by turbulent mixing, entrainment, rotation, and the presence of a low-level jet. In order to isolate blockage effects, we compared the first row of a wind farm to a solitary row of turbines. We observed that the first row of a wind farm does not receive undisturbed inflow, as we also demonstrated in Chapters 4 and 5 and has been

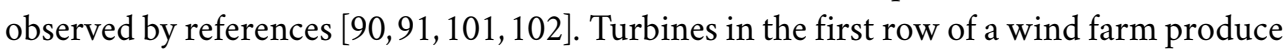
less power when downstream turbines are placed more closely and this effect is amplified when the atmosphere is more stably stratified. The magnitude of the blockage effect is less than that of wake effects, but should still be considered in wind farm planning.

We confirmed that the power reduction in the first row is due to induction. When compared to a solitary row of turbines, the first row of a wind farm produces less power because incoming airflow is inclined to pass over the turbine array as opposed to through it. We observed that a wind turbine array acts as an obstruction to incoming flow, exhibited by a slow-down of the wind at hub-height in the induction region as flow is deflected upwards. Both the distance between downstream turbines and thermal stratification are key factors influencing the incoming airflow. The velocity reduction preceding the array is more pronounced and extends farther upstream when the streamwise turbine spacing decreases. Additionally, we observed that induction was further exacerbated by increasing stability, despite the fact we do not impose a capping inversion and no gravity waves are present [115 118]. Enhanced induction in stable conditions was also observed by Gomez et al. [133] who attributed this trend to changes in turbulent vertical motions. We show that the behavior observed in the induction region is related to the inherent adverse pressure gradient which is stronger when the turbine density or stable stratification increases. Overall, these upstream effects, and their dependence on turbine spacing and atmospheric stability, influence power output and should be considered when making measurements or predictions for the region at the entrance row of a wind farm.

More research is required to better understand how blockage is impacted by thermal stability. For instance, this analysis can be furthered by considering the turbulence energetics involved [152]. We also recommend that future studies should consider a solitary turbine as a reference case, accompanied by even more realistic conditions such as finite-size arrays. Additionally, varying the spanwise turbine spacing will influence how wind flows around the turbines. Nonetheless, we have shown that flow blockage can influence wind farms, especially when the atmosphere is stably stratified. 
CHAPTER 7
Conclusions and outlook

CHAPTER 7
Conclusions and outlook

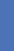

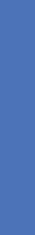

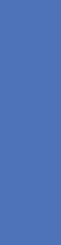

111

Conclusi

\section{CHAPTER 7}

\section{a}

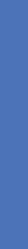

(?)

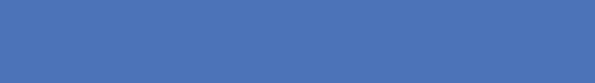

P

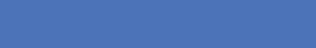

(

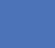

(n)

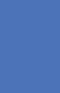
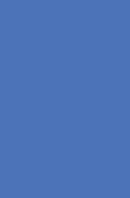
112

CONCLUSIONS AND OUTLOOK 
Satisfying our global energy needs with sustainable sources is necessary in combating humandriven climate change. As the wind energy industry thrives, optimizing the power produced by wind farms becomes increasingly relevant. Models and simulations based on turbulent physics are powerful tools for predicting wind farm performance. This thesis uses these techniques to better understand the downstream and upstream phenomenon which influence turbine productivity. Part I focused on reducing downstream power losses related to wakes by vertically staggering the wind turbines. Part II shed light on the relatively unexplored upstream influence of turbines, known as blockage, and how it impacts wind farm performance.

Chapter 2 explored the potential benefit of vertically staggered wind farms using an analytical model. A well-established top-down model [16 18.37. was further developed and applied to study the scenario in which relatively short turbines are placed within a fixed array of taller turbines. The results showed that adding short turbines to an existing array of tall turbines increases the wind farm's total power production. Moreover, the power contribution of the short turbines outweighs the hindrance of increased wake interactions. This simple model generates an abundance of results quickly, allowing the relative importance of wind farm design parameters to be investigated. The results indicated that the benefit of vertical staggering is influenced by the short turbine density (number per tall turbine), hub-height, and diameter, as well as the surface roughness scale. However, wind farm planning considers both the productivity and accompanying costs of a potential wind farm. Therefore, a simplified cost analysis was performed to verify if the power contribution offsets the expenses of the additional turbines. The results suggested that this method of vertical staggering may not be profitable, but this depends on the particular characteristics of the wind farm. More research is recommended to consider vertical staggering as a viable option in wind farm design. Future studies should focus on supplementing and further validating this model using high-fidelity models.

Of course, many interesting scenarios remain and can be explored using this analytical model. For instance, as the technology advances, adding taller turbines to a fixed array of 'short' turbines may be more applicable to retro-fitting existing wind farms with turbines that are even larger than current turbines. Also, this model can be applied to new vertically staggered wind farms: a scenario in which both the short and tall turbine spacing can be simultaneously adjusted and optimized. The model itself can also be further enhanced. For instance, incorporating thermal stratification or finite-size arrays with downstream development would make the model more realistic 31, 32, 56. However, as is, the model could not distinguish the relative positioning of the short turbines, which is therefore addressed in the following chapter using large eddy simulations. Nonetheless, basic trends were established and useful insight was obtained for future studies. 

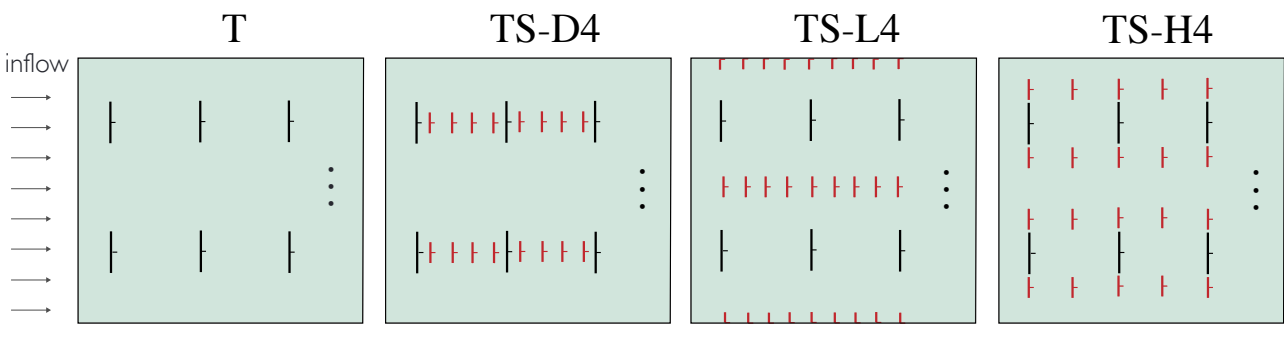

Figure 7.1: Diagram of the reference wind farm with only tall turbines (T) and the vertically staggered wind farms (TS) with varied relative positioning of the short turbines (red).

Chapter 3 explored vertically staggered wind farms using more accurate, high-fidelity large eddy simulations. In this chapter, large eddy simulations are described in detail, showcasing the basics of the wind farm analysis which is also applied in Part II. Parallel to the previous chapter, the scenario in which short turbines are added to a fixed array of tall turbines is considered. Limited cases could be explored due to the high computational cost of these simulations. Therefore, insight from the analytical model is used, focusing on the effect of the short turbine density and the surface roughness scale. Additionally, a key advantage of the large eddy simulations is that the importance of the relative position of the short turbines can be investigated. Therefore, short turbines were added to a reference case, placing them either downstream or laterally in between the tall turbine columns. In agreement with Chapter 2 the results convincingly show that the short turbines contribute more than they hinder to the total power production. In fact, the cumulative power gain $\left(\Delta P=\left(P_{\text {cum }, \mathrm{TS}}-P_{\mathrm{cum}, T}\right) / P_{\mathrm{cum}, T}\right)$ was higher even when four short turbines were added directly downstream of each tall turbine. Nonetheless, the largest gain was observed in offshore conditions and when short turbines were placed between the tall turbine columns, benefiting from horizontal staggering as well.

Moreover, in Chapter 3 the vertical and lateral alignment of the short turbines with respect to the tall turbines was adjusted; however, the short turbines were always placed directly downstream with respect to each other (refer to Figure 7.1 TS-D4, TSL4). Offsetting the short turbines with respect to each other could reduce their wake interactions, which is briefly explored here with an additional TS-H4 layout. Figure 7.2 shows the time- and horizontally-averaged streamwise velocity profile for each of the configurations illustrated in Figure 7.1 The velocity at hub-height is lower for the TS-H4 layout, indicating that the short turbines are extracting more momentum than when they are placed in-line with each other. However, the short turbines in the TS-H4 layout may impact the performance of the tall turbines more due to their closer proximity. Therefore, in Table 7.1 the cumulative power gain of the TS- $\mathrm{H} 4$ case is compared to the 


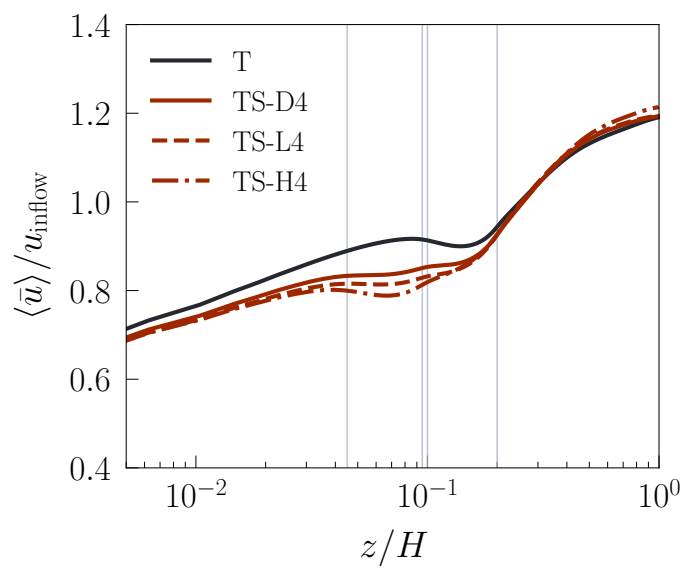

Figure 7.2: Time- and horizontally-averaged streamwise velocity profile as a function of height for the wind farm where $z_{0} / H=10^{-6}, H=1000 \mathrm{~m}$, and $s_{T}^{2}=5.24 \times 7.85$. Vertical gray lines indicate the range of the rotor-swept areas of short $\left(H_{S}-D_{S} / 2 \leqslant z \leqslant H_{S}+D_{S} / 2\right)$ and tall turbines $\left(H_{T}-D_{T} / 2 \leqslant z \leqslant H_{T}+D_{T} / 2\right)$.

TS-D4 and TS-L4 results of Chapter 3 Despite their negative impact on the tall turbines, the short turbines in this layout contribute more to the total wind farm power output resulting in the highest gain due to the fact that the short turbines experience less wake interactions. Nonetheless, TS-H4 is not necessarily the optimal configuration, and future studies should consider layouts beyond the limited cases presented in this thesis. Short turbine placement becomes a more complex challenge with more degrees of freedom and requires further research.

\begin{tabular}{ccc}
\hline Layout & $\Delta P\left(z_{0} / H=10^{-4}\right)$ & $\Delta P\left(z_{0} / H=10^{-6}\right)$ \\
\hline TS-D4 & $37.8 \%$ & $44.2 \%$ \\
TS-L4 & $48.3 \%$ & $54.9 \%$ \\
TS-H4 & $58.1 \%$ & $68.8 \%$ \\
\hline
\end{tabular}

Table 7.1: Cumulative power gain of vertically staggered layouts (TS) compared to the reference case without short turbines $(\mathrm{T})$. Onshore and offshore conditions correspond to the roughness scales $z_{0} / H=10^{-4}$ and $z_{0} / H=10^{-6}$, respectively. 
(a)

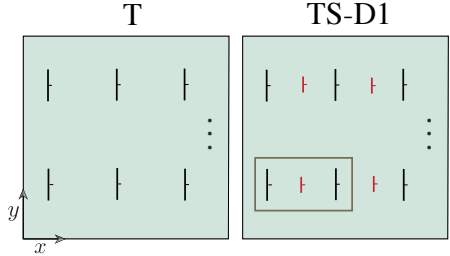

(b)

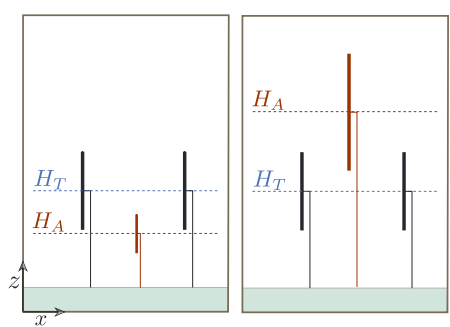

$(c)$

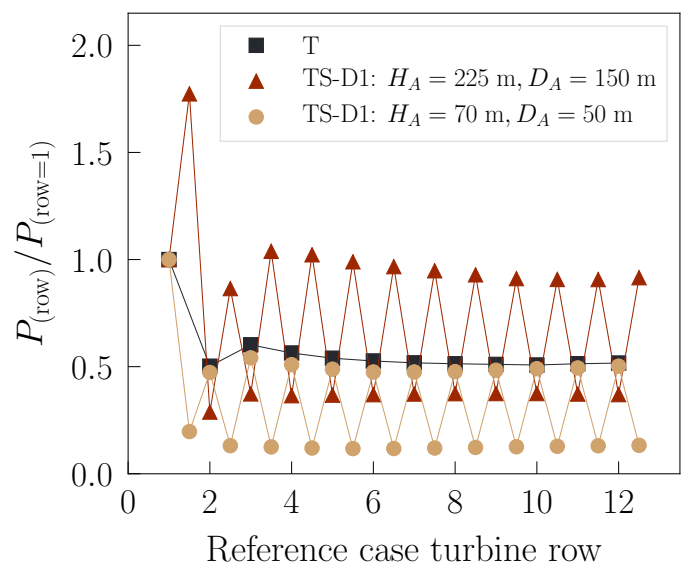

Figure 7.3: Sketches depicting (a) the reference layout ( $\mathrm{T}$ ) with only tall turbines and vertically staggered layout (TS-D1) with added turbines (red) and (b) the two options for added turbines. (c) Average power output of wind turbine rows in offshore conditions normalized by the first row for the reference case and the two vertically staggered wind farms where the added downstream turbines are either larger or smaller $\left(H_{A}, D_{A}\right)$.

The technology continues to develop such that turbines are becoming even larger and more productive [4]. With this in mind, it may be someday feasible to create vertically staggered wind farms by retro-fitting existing wind farms with new, taller turbines. This approach would take advantage of the higher velocity wind above current wind farms while not requiring extra land. Therefore, in this outlook a scenario is briefly explored where even taller turbines $\left(H_{A}=225 \mathrm{~m}, D_{A}=150 \mathrm{~m}\right)$ are added to the reference case $\left(H_{T}=150 \mathrm{~m}, D_{T}=100 \mathrm{~m}\right)$, as illustrated in Figure $7.3 \mathrm{a}$ ) and (b). In contrast to the original configuration with short additional turbines, this new scenario has significant vertical overlap and therefore higher wake exposure. The average power of each row normalized by the first row for both vertically staggered wind farms (TS-D1) is shown in Figure 7.3 c) alongside the reference wind farm (T). The figure shows that the first row of the reference-size turbines, which is not exposed to wake, produces less power than the even taller turbines added downstream. In fact, the first row of even taller turbines produces almost twice as much power. This result is not unexpected because vertical staggering is quite effective at the front of the farm, the wind is stronger, higher up in the atmosphere, and the turbine rotor-size is $2.25 \times$ larger. Nonetheless, these exceptionally tall turbines generate and experience substantial wake interactions, 
such that this assigned spacing is likely not ideal. The increased wake also hinders the performance of the reference-size turbines. Specifically, the reference-size turbines in the vertically staggered farm deviate farther from the reference case when the even taller turbines are added as opposed to the smaller turbines. However, when the even taller turbines are added to the reference layout, the cumulative power gain is $\Delta P=148.8 \%$ compared to $\Delta P=16.5 \%$ when the short turbines are added. Notwithstanding, the difference in installed capacity and costs of implementation would be vastly different. Therefore, although this brief exploration provides some intriguing insight, it would be useful in future studies to compare the productivity of reference wind farm cases in which the additional turbines are alone, as well as the accompanying costs.

Chapter 4 substantiated wind farm blockage in a neutral atmospheric boundary layer (ABL) using large eddy simulations. Flow blockage is the influence that an upcoming obstacle has on the inflow and is only recently gaining attention in relation to wind farms. Therefore, assuming that the first row of a wind farm receives undisturbed flow was sufficient when analyzing downstream wake effects, but this assumption is not comprehensively valid due to upstream blockage effects. In this study, spanwise and streamwise blockage effects were distinguished by comparing a stand-alone turbine, a solitary infinite row of turbines, and an entire wind farm with eight rows. This comparison showed that due to the deflection of incoming flow, close spanwise-neighboring turbines can benefit each other while close downstream turbines hinder the performance of the first row of a wind farm. By adjusting the spanwise and streamwise turbine spacing, it became obvious that blockage increases when spacing decreases and is most prominent in dense turbine arrays $(s<4)$. These results are in good agreement with recent wind tunnel experiments [90] and simulations [99, 113, 123], but this study emphasizes that wind farm blockage is highly dependent on the specific turbine layout. Additionally, the blockage effect appeared to be influenced by the turbine hub-height, but was not dependent on the turbulence intensity at hub-height indicating that blockage is a nonturbulent phenomenon. The potential impact of the spanwise row length on the blockage effect was briefly examined, finding that the magnitude of the effect decreases when the rows are finite but that the overall trends remain the same. Blockage exists and though the magnitude of the effect is less substantial than wake-related power losses, this effect should be considered in wind farm planning and the first row of a wind farm is not a perfect reference.

This research focused on the performance of the first row where blockage effects are most obvious. However, if and how consecutive rows are potentially affected by blockage caused by downstream turbines is another compelling topic that should be investigated. Here, this topic is briefly addressed by comparing four wind farms with the same layout $\left(s_{y}=1.57, s_{x}=1.96, C_{T}^{\prime}=4 / 3\right)$ while adjusting the number of downstream rows $\left(N_{T, x}=8,4,2\right)$. The second row in the $N_{T, x}=2$ configuration 
is subjected to wakes but does not experience blockage due to downstream turbines. So, using this second row as a reference case $P_{2(\mathrm{row}=2)}$, the performance of the second row of each wind farm is compared to get an idea of blockage implications throughout the wind farm. The normalized power output of the second row in the wind farm with four rows is $P_{4(\text { row }=2)} / P_{2(\text { row }=2)}=0.93$. Therefore, the second row in a wind farm with only two rows performs better than when two additional rows are placed closely downstream. When four more rows are added, and there are eight rows in total, the relative performance of the second row decreases further to $P_{8(\mathrm{row}=2)} / P_{2(\mathrm{row}=2)}=$ 0.90 due to increased blockage from downstream turbines. Further investigation is required to better understand the effects of blockage throughout the wind farm which may unknowingly induce power losses.

Chapter 5 focused on how the blockage effects, established in the previous chapter, are dependent on the turbine thrust coefficient. The thrust coefficient was systematically varied while comparing a stand-alone turbine, a solitary infinite row of turbines, and a wind farm with eight rows. The same trends for spanwise and streamwise blockage effects were observed, but also the blockage was clearly dependent on the thrust coefficient. Specifically, the spanwise blockage effect appeared to have a linear relationship with the disk-based thrust coefficient $C_{T}^{\prime}$, such that the relative power of turbines within a row perform increasingly better than a stand-alone turbine as $C_{T}^{\prime}$ increases. On the other hand, the streamwise blockage effect appeared to decrease linearly with the thrust coefficient $C_{T}$, such that the relative power of the first row of a wind farm normalized by the solitary row decreases as $C_{T}$ increases. Overall, this chapter established that as the thrust force of each turbine increases, the magnitude of the blockage they induce will increase accordingly.

Chapter 6 investigated flow blockage in a stably stratified ABL. A stable temperature profile limits turbulent motions which reduces wake recovery, and exacerbates blockage caused by downstream turbines. The large eddy simulations were adapted to include buoyancy and rotational forces. Specifically, streamwise blockage effects were examined in a stable ABL by comparing the first row of a wind farm to a solitary infinite row while systematically varying the streamwise turbine spacing and the surface cooling rate. The results showed that when turbines are placed more closely downstream, or stable stratification increases, the velocity preceding the wind farm at hub-height decreases as more air is deflected over the wind farm as opposed to passing through it. Therefore, turbines at the entrance of the wind farm produce less power than they would without downstream turbines or in the absence of thermal stratification.

The computational cost of the simulations was even higher than the cases without thermal stratification and therefore less scenarios could be investigated. Future studies should also consider the effect of the spanwise turbine spacing. Although not presented here, additional simulations indicated that blockage may be reduced for select cases 
where the spanwise spacing is larger and the wind flows more readily between the rows. However, more research is required to draw any conclusions. Furthermore, the benefit previously observed from close spanwise neighboring turbines in neutral ABL should also be explored in a stable ABL. Therefore, future studies should incorporate spanwise-finite layouts and a stand-alone turbine as a reference case. Nonetheless, we have shown that blockage should be considered in wind farm planning, especially in stable ABL conditions.

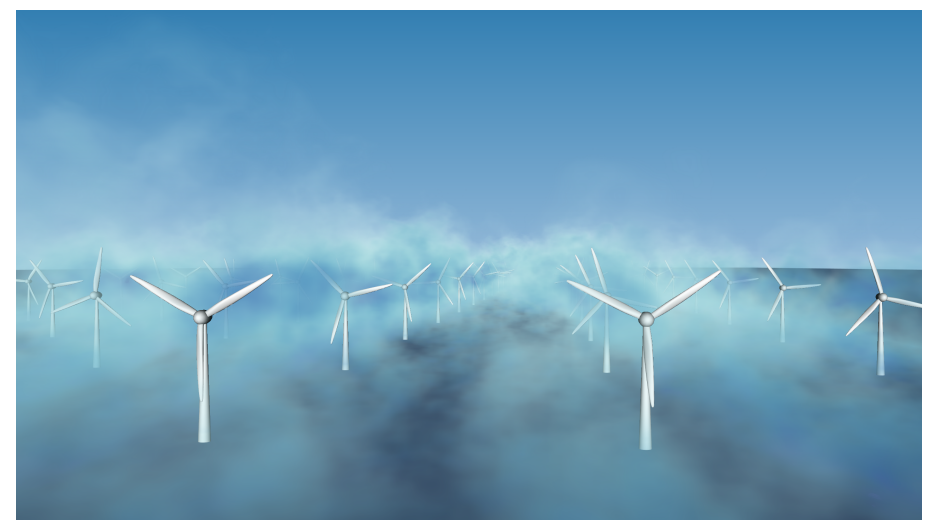

Figure 7.4: Visualization of one of our large eddy simulations by Dr. Cristian C. Lalescu. 


\section{Summary}

In spite of the current climate crisis, our expanding population is demanding more energy resources. However, reducing our carbon emissions is imperative in mitigating humandriven climate change, therefore necessitating the development of sustainable sources such as wind energy. Maximizing the productivity of wind farms while minimizing the space that they occupy requires understanding the turbulent fluid physics involved. For instance, each turbine generates a slowed, yet more turbulent flow downstream, known as a wake. Also, turbines influence the incoming flow through blockage. Therefore, the interactions between these turbines and the atmosphere will determine the productivity of the entire wind farm. Modeling and numerical simulations are excellent tools for understanding and optimizing wind farms prior to construction. In this thesis, a simple analytical model and high-fidelity large eddy simulations (LES) are used to investigate the effects of wakes and blockage on the productivity of large-scale wind farms.

In Part I of this thesis, we address wake-related power losses. Specifically, we explore vertically staggering wind turbines as a method to circumvent negative wake effects and take advantage of relatively undisturbed flow. In each chapter, we examine the effectiveness of adding short turbines to a fixed array of tall turbines. First, in Chapter 2 an analytical top-down model is further developed and applied to vertically staggered wind farms. Though simple, the readiness of this model elucidates basic trends, showing that the potential benefit of vertically staggered wind farms is highly dependent on the wind turbine height, diameter and spacing, as well as the surface roughness scale. We observe that adding relatively short turbines to a wind farm can increase the wind farm's total productivity, depending on the aforementioned variables. Furthermore, although more productive, our simplified cost analysis reveals that such a configuration may not be more profitable. Sometimes LES is more. Therefore, in Chapter 3 more accurate LES are performed, using insight from the analytical model, to better understand when vertical staggering is more productive and the sensitivity to these parameters. Good agreement between the analytical model and LES is found when comparing the cumulative gain in power. Also, both the analytical model and LES suggest that vertical staggering is more beneficial in offshore conditions. However, in contrast to the analytical model, 
the LES could distinguish the relative position of the added turbines which is shown to substantially impact their power contribution. For instance, adding short turbines can be more even more advantageous when also horizontally staggered and when the first row is not exposed to wakes. Nonetheless, the expenses which accompany each wind farm configuration must be accounted for in order to further asses the advantage of vertical staggering in wind farm design.

In Part II of this thesis, we investigate a relatively unexplored phenomenon in wind farms: flow blockage. Although assuming that the first row of a wind farm receives undisturbed flow is acceptable when studying downstream phenomenon such as wakes, this assumption is generally not valid due to upstream blockage effects. In Chapters 4 and 5. we study blockage using LES in a neutral atmospheric boundary layer. We consider both spanwise and streamwise blockage by comparing a stand-alone turbine, an infinite row of turbines and a wind farm with eight rows. We observe that the slow-down of wind preceding the turbine arrays, known as induction, is exacerbated when turbines are placed close together compared to the stand-alone case. Furthermore, flow deflection allows close spanwise neighboring turbines to benefit from each other. Meanwhile, close downstream turbines hinder the first row of a wind farm as the flow is inclined to pass over the array. We systematically explore the effect of multiple parameters such as the inter-turbine spacing, hub-height, thrust coefficient, and the turbulence intensity at hub-height. We determine that blockage effects are highly dependent on the turbine spacing and thrust coefficient. Essentially, blockage increases when turbines are placed closer together and their thrust increases. However, the atmospheric boundary layer is not commonly neutral, so in Chapter 6 , we execute LES in a stable boundary layer while systematically varying the surface cooling rate. In doing so, we also observe that blockage increases when the atmosphere is stably stratified. The ambiguity and lack of consensus that still surrounds this phenomenon maintains that blockage is still relatively unaccounted for when designing wind farms.

In Chapter 7 we provide more detailed conclusions and perspectives than presented here. As wind farms become increasingly prevalent and turbines become larger, understanding wakes and blockage will be even more pertinent in next generation wind farms. For instance, we briefly entertain the feasibility of vertical staggering by retro-actively placing even larger turbines into a wind farm. Also, we have established that the first row of a wind farm does not receive undisturbed flow, however, we also broach blockage effects throughout the wind farm. Many compelling questions remain regarding wakes and blockage in large-scale wind farms and I hope that this thesis inspires future work. 


\section{Samenvatting}

Samenvatting door Jessica Strickland: Het klimaat is kapot. Windparken helpen daarbij. Dit boek beschrijft hoe ze dat doen. Windturbines maken elektriciteit uit wind en in principe moet je windturbines niet zo dicht bij elkaar plaatsen. Het eind (kwak).

Vertaling door Maaike Rump: Ondanks de klimaatcrisis vraagt de groeiende bevolking van de wereld steeds naar meer stroom. Ondertussen is het van groot belang dat de uitstoot van broeikasgassen vermindert, waardoor het noodzakelijk is om de ontwikkelingen in duurzame energiebronnen, zoals windturbines, te stimuleren. Om de productiviteit van windmolenparken te verhogen op een zo'n klein mogelijk oppervlak vereist begrip van de betrokken turbulente luchtstromingen. Een voorbeeld is de vertraging in de snelheid van de luchtstroom achter een windturbine, oftewel de windschaduw. Ook blokkeren de turbines de wind van voren. Daarom zijn het de interacties tussen de windturbines en de atmosfeer die de productiviteit van het gehele windmolenpark bepalen. Het modelleren van zulke parken is een ideale methode om de windmolenparken te begrijpen en optimaliseren voordat deze gebouwd worden. In dit proefschrift worden een simpel analytisch model en large eddy simulaties (LES) gebruikt om de invloeden te bestuderen van de windschaduw en het blokkeren van de luchtstroom op de productiviteit van grootschalige windmolenparken.

In Deel 1 van dit proefschrift richten we ons op het verlies in vermogen door effecten van de windschaduw. Om precies te zijn, we kijken naar de mogelijkheid om verschillende hoogtes van turbines te gebruiken om negatieve invloeden van de windschaduw te vermeiden en voordeel te halen uit de daardoor onverstoorde luchtstroom. In elk hoofdstuk onderzoeken we hoe voordelig het zou zijn om lagere windturbines toe te voegen aan een bepaalde reeks van hogere turbines. Als eerste, in Hoofdstuk 2, is een top-down analytisch model verder ontwikkeld en is het toegepast op een windmolenpark met verschillende turbinehoogtes. $\mathrm{Al}$ is het eenvoudig, dit bestaande model licht simpele trends toe, waardoor duidelijk is geworden dat de mogelijke winst van verschillende turbineshoogtes gebruiken zeer afhankelijk is van de hoogte en diameter van de turbine, afstand tussen turbines, en de schaal van ruwheid van het oppervlak. De resultaten laten 
zien dat relatief korte turbines toevoegen aan een windmolenpark kan de totale productiviteit verhogen, rekening houdend met de variabelen die hiervoor benoemd zijn. Daarnaast geeft de analyse van kosten, ondanks de verhoogde productiviteit, wel aan dat dit niet per se tot meer winst leidt. In Hoofdstuk 3 worden LES met meer detail gebruikt, met behulp van wat er is geleerd uit het eenvoudige model, om beter te begrijpen wanneer de verschillende turbinehoogtes productief zijn en hoe gevoelig dit is voor de gebruikte variabelen. Er is een goede overeenkomst tussen het analytisch model en de LES als het aankomt op de totale toename aan stroom. Daarnaast geven zowel het analytisch model en de LES indicaties dat het gebruik van verschillende hoogtes van windturbines een groter voordeel heeft als het windmolenpark in de zee staat. Daarentegen laten de resultaten van de LES zien, in tegenstelling tot het analytisch model, wat de invloed is van de relative positie van de toegevoegde lagere turbines, waaruit blijkt dat dit een grote invloed heeft op de totale stroom die gemaakt kan worden. Bijvoorbeeld, het toevoegen van lagere turbines is nog voordeliger als de windturbines niet in dezelfde lijn achter elkaar staan zolang the voorste rij niet in de windschaduw staat. Maar om duidelijk het voordeel in te kunnen schatten van verschillende turbinehoogtes, moeten ook de constructiekosten worden meegenomen voor de verschillende ontwerpen.

In Deel 2 van dit proefschrift bestuderen we een nog vrij onbekend fenomeen voor windmolenparken, namelijk het blokkeren van de luchtstroom door de turbines. Ook al wordt het geaccepteerd dat de voorste rij in een windmolenpark een onverstoorde luchtstroom toegevoerd krijgt om bijvoorbeeld de windschaduw te bestuderen, klopt deze aanname eigenlijk niet door het blokkeringseffect op de inkomende luchtstroom. In Hoofdstuk 4 en 5 bestuderen we dit blokkeringseffect met gebruik van LES in een neutrale atmosferische grenslaag. Hierbij nemen we zowel blokkering loodrecht als stroomsgewijs mee door te kijken naar zowel een alleenstaande turbine, als een oneindige rij van turbines, en een windmolenpark van acht rijen. De resultaten laten zien dat luchtstroom vertraagd voordat het bij de turbine aankomt, en dat dit wordt versterkt als meerdere turbines dicht naast elkaar staan. Ook heeft een windturbine voordeel van zijn buren door de afgebogen luchtstroom van de andere turbine. Maar een turbine dicht achter deze eerste rij staat werkt weer in het nadeel voor de eerste rij doordat de luchtstroom meer geneigd is om over de rij heen te gaan. We hebben systematisch het effect van meerdere parameters bestudeerd, zoals de afstand tussen turbines, de hoogte van de turbine, de stuwkracht, en de intensiteit van de turbulentie ter hoogte van de turbine. Hieruit is gekomen dat het blokkeringseffect het meest wordt beïnvloed door de afstand tussen turbines en de stuwkracht. Wat we zien is dat de blokkering toeneemt als de turbines dicht bij elkaar staan en de stuwkracht toenemend. Er moet wel rekening gehouden worden met dat de atmosferische grenslaag meestal niet neutraal is. Daarom beschrijft Hoofdstuk 6 LES in een stabiele grenslaag waar systematisch de afkoeling van het oppervlak wordt verandert. Hierdoor zien we ook dat de blokkering toeneemt zodra de atmosfeer een gelaagde 
stroom is. Dat er rondom het bltokkeringsfenomeen nog veel onenigheid is, wordt het vaak niet meegenomen bij het ontwerpen van een windmolenpark.

In Hoofdstuk 7 gaan we in op meer details van de conclusies en perspectieven die zien getrokken. Doordat er steeds meer windmolenparken zijn met grotere turbines is het essentieel om de invloed van de windschaduw en het blokkeringseffect te begrijpen. We kunnen bijvoorbeeld achteraf nog grotere windturbines plaatsen in een bestaand park, om de voordelen van de hoogteverschillen te gebruiken. We hebben ook laten zien dat de voorste rij van een windmolenpark geen onverstoorde luchtstroom toegevoerd krijgt, al beginnen we ook over het blokkeringseffect binnenin het park. Er zijn nog veel vragen die beantwoord moeten worden over de windschaduw en de windblokkering in een groot windmolenpark, en hopelijk is deze thesis een inspiratie. 



\section{References}

[1] I. Eames and J. B. Flor, "New developments in understanding interfacial processes in turbulent flows”, Phil. Trans. Soc. A 369, 702-705 (2011), doi:10.1098/rsta.2010.0332

[2] J. J. Monaghan and J. B. Kajtar, "Leonardo da Vinci's turbulent tank in two dimensions", European Journal of Mechanics - B/Fluids 44, 1-9 (2014), doi:10.1016/j.euromechflu.2013.09.005

[3] V. Masson-Delmotte, A. P. P. Zhai, S. B. S. L. Connors, C. Péan, N. Caud, Y. Chen, L. Goldfarb, M. I. Gomis, M. Huang, K. Leitzell, E. Lonnoy, J. B. R. Matthews, T. K. Maycock, T. Waterfield, O. Yelekçi, R. Y. (ed.), and B. Z. (ed.), "Summary for policymakers. in: Climate change 2021: The physical science basis. contribution of working group $i$ to the sixth assessment report of the intergovernmental panel on climate change", Technical Report, Intergovernmental Panel on Climate Change (2021), cambridge University Press. In Press.

[4] Global Wind Energy Council, "Global wind energy report 2021", (2021).

[5] D. G. Shepherd, "Historical development of the windmill", National Aeronautics and Space Administration Contractor Report 4337 (1990), doi:10.2172/6342767

[6] M. Pasqualetti, R. Righter, and P. Gipe, "History of wind energy", Elsevier Encyclopedia of Energy 6, 419-433 (2004), doi:10.1016/B0-12-176480-X/00045-0

[7] A. Lucas, Wind, Water, Work: Ancient and Medieval Milling Technology (Brill Academic Publishers) (2006), ISBN: 978-90-04-14649-5

[8] www.theguardian.com, "Dutch electric trains become $100 \%$ powered by wind energy”, The Guardian (2017).

[9] A. Betz, Introduction to the Theory of Flow Machines (Elsevier) (1966), ISBN:9781483180908 
[10] F. Porté-Agel, C. Meneveau, and M. B. Parlange, "A scale-dependent dynamic model for large-eddy simulation: application to a neutral atmospheric boundary layer", J. Fluid Mech. 415, 261-284 (2000), doi:10.1017/S0022112000008776

[11] R. J. A. M. Stevens, M. Wilczek, and C. Meneveau, "Large-eddy simulation study of the logarithmic law for second and higher-order moments in turbulent wall-bounded flow", Journal of Fluid Mechanics 757, 888-907 (2014), doi:10.1017/jfm.2014.510

[12] N. O. Jensen, "A note on wind generator interaction", Risø-M-2411, Risø National Laboratory, Roskilde (1983), ISBN:87-550-0971-9

[13] J. Choi and M. Shan, "Advancement of Jensen (PARK) wake model", EWEA Conference, Vienna, February 2013 (2013), ISBN: 978-1-63266-314-6.

[14] A. Peña, P. E. Réthoré, and O. Rathmann, "Modeling large offshore wind farms under different atmospheric stability regimes with the Park wake model", Renewable Energy 70, 164-171 (2014), doi:10.1016/j.renene.2014.02.019

[15] N. O. Jensen, "Change of surface roughness and the planetary boundary layer", Q. J. R. Meteorol. Soc. 104, 351-356 (1978), doi:10.1002/qj.49710444009

[16] S. Frandsen, "On the wind speed reduction in the center of large clusters of wind turbines", J. Wind Eng. Ind. Aerodyn. 39, 251-265 (1992), doi:10.1016/01676105(92)90551-K

[17] S. Frandsen, R. J. Barthelmie, S. Pryor, O. Rathmann, S. Larsen, J. Højstrup, and M. Thøgersen, "Analytical modeling of wind speed deficit in large offshore wind farms", Wind Energy 9, 39-53 (2006), doi:10.1002/we.189

[18] M. Calaf, C. Meneveau, and J. Meyers, "Large eddy simulation study of fully developed wind-turbine array boundary layers”, Phys. Fluids 22, 015110 (2010), doi:10.1063/1.3291077

[19] S. B. Pope, Turbulent Flows (Cambridge University Press, Cambridge) (2000), doi:10.1017/CBO9780511840531.

[20] P. Davidson, Turbulence: An Introduction for Scientists and Engineers (Oxford University Press, Oxford) (2004), ISBN-13:9780198722588.

[21] B. Cushman-Roisin and J. Beckers, Introduction to Geophysical Fluid Dynamics: Physical and numerical aspects, 2nd edition (Academic Press) (2011), ISBN9780120887590 
[22] J. Wieringa, A. G. Davenport, C. S. B. Grimmond, and T. R. Oke, "New revision of davenport roughness classification”, 3EACWE (3rd European \& African Conference on Wind Engineering), Eindhoven, The Netherlands (2001), doi:10.1016/01676105(92)90434-C

[23] F. Porté-Agel, M. Bastankhah, and S. Shamsoddin, "Wind-turbine and wind-farm flows: A review”, Boundary-Layer Meteorol. 74, 1-59 (2020), doi:10.1007/s10546019-00473-0

[24] P. K. Kundu and I. M. Cohen, Fluid mechanics (Elsevier) (2001), ISBN:9780124059351

[25] O. Reynolds, "An experimental investigation of the circumstances which determine whether the motion of water shall be direct or sinuous and of the law of resistance in parallel channels", Phil. Trans. R. Soc. A 174, 935-982 (1883), doi:10.1098/rstl.1883.0029.

[26] D. Zoric and V. A. Sandborn, "Similarity of large reynolds number boundary layers", Boundary-Layer Meteorology 2, 326-333 (1972), doi:10.1007/BF02184773

[27] D. Mehta, A. H. van Zuijlen, B. Koren, J. G. Holierhoek, and H. Bijl, "Large eddy simulation of wind farm aerodynamics: A review", J. Wind Eng. Ind. Aerodyn. (2014), doi:10.1016/j.jweia.2014.07.002

[28] J. Smagorinsky, "The role of numerical modeling", Bull. Am. Meteorol. Soc. 48, 89-93 (1967), url: jstor.org/stable/26249982.

[29] D. K. Lilly, "The representation of small-scale turbulence in numerical simulation experiments", Proc. IBM Sci. Comp. Symp. on Environmental Sciences (1967), doi:10.5065/D62R3PMM

[30] R. B. Smith, "Gravity wave effects on wind farm efficiency", Wind Energy 13, 449458 (2009), doi:10.1002/we.366

[31] C. Meneveau, "The top-down model of wind farm boundary layers and its applications", J. Turb. 13, 1-12 (2012), doi:10.1080/14685248.2012.663092

[32] R. J. A. M. Stevens, "Dependence of optimal wind-turbine spacing on wind-farm length”, Wind Energy 19, 651-663 (2016), doi:10.1002/we.1857

[33] L. P. Chamorro, R. E. A. Arndt, and F. Sotiropoulos, "Turbulent flow properties around a staggered wind farm", Boundary-Layer Meteorol. 141, 349-367 (2011), doi:10.1007/s10546-011-9649-6. 
[34] Y. T. Wu and F. Porté-Agel, "Simulation of turbulent flow inside and above wind farms: Model validation and layout effects", Boundary-Layer Meteorol. 146, 181205 (2013), doi:10.1007/s10546-012-9757-y

[35] R. J. A. M. Stevens, D. F. Gayme, and C. Meneveau, "Effect of turbine alignment on the average power output of wind-farms”, ICOWES 2013 611-623 (2013).

[36] R. J. A. M. Stevens, D. F. Gayme, and C. Meneveau, "Large eddy simulation studies of the effects of alignment and wind farm length”, J. Renew. Sustain. Energy 6, 023105 (2014), doi:10.1063/1.4869568

[37] S. Xie, C. L. Archer, N. Ghaisas, and C. Meneveau, "Benefits of collocating verticalaxis and horizontal-axis wind turbines in large wind farms”, Wind Energy 20, 45-62 (2017), doi:10.1002/we.1990

[38] T. Chatterjee and Y. T. Peet, "Exploring the benefits of vertically staggered wind farms: Understanding the power generation mechanisms of turbines operating at different scales”, Wind Energ. 22, 283-301 (2018), doi:10.1002/we.2284

[39] M. Zhang, M. G. Arendshorst, and R. J. A. M. Stevens, "Large eddy simulations of the effect of vertical staggering in large wind farms", Wind Energy 22, 189-204 (2019), doi:10.1002/we.2278.

[40] C. Koning, "Influence of the propeller on other parts of the airplane structure", in Aerodynamic Theory, 361-430 (Springer) (1935), doi:10.1007/978-3-642-91487-44

[41] H. Glauert, Airplane Propellers Aerodynamic Theory Volume IV edited by William Frederick Durand (The Dover edition) (1963), doi:10.1007/978-3-642-91487-4

[42] M. Zhang and R. J. A. M. Stevens, "Exploring a better turbine layout in vertically staggered wind farms”, J. Phys. Conf. Ser. 1037, 072041 (2018), doi:10.1088/17426596/1037/7/072041

[43] Y. Wu, T.-L. Liao, C.-K. Chen, C.-Y. Lin, and P.-W. Chen, "Power output efficiency in large wind farms with different hub heights and configurations", Renewable Energy 132, 941-949 (2019), doi:10.1016/j.renene.2018.08.51

[44] M. H. Vested, N. Hamilton, J. N. Sørensen, and R. B. Cal, "Wake interaction and power production of variable height model wind farms”, J. Phys. Conf. Ser. 1012169 (2014), doi:10.1088/s1742-6596/524/1/012169

[45] L. P. Chamorro, N. Tobin, R. E. A. Arndt, and F. Sotiropoulos, "Variable-sized wind turbines are a possibility for wind farm optimization”, Wind Energy 10, 1483-1494 (2014), doi:10.1002/we.1646. 
[46] Y. Chen, H. Li, K. Jin, and Q. Song, "Wind farm layout optimization using genetic algorithm with different hub height wind turbines", Energy Convers. Manag. 70, 56-65 (2013), doi:10.1016/j.enconman.2013.02.007

[47] K. Chen, M. X. Song, X. Zhang, and S. F. Wang, "Wind turbine layout optimization with multiple hub height wind turbines using greedy algorithm”, Renewable Energy 96, 676-686 (2016), doi:10.1016/j.renene.2016.05.018

[48] L. P. Chamorro, R. E. A. Arndt, and F. Sotiropoulos, "Turbulence within variablesize wind turbine arrays", J. Phys. Conf. Ser. 555, 012098 (2014), doi:10.1088/17426596/555/1/012098.

[49] L. Wang, A. C. C. Tan, M. Cholette, and Y. Gu, "Comparison of the effectiveness of analytical wake models for wind farm with constant and variable hub heights", Energy Conservation and Management 124, 189-202 (2016), doi:10.1016/j.enconman.2016.07.017

[50] B. DuPont, J. Cagen, and P. Moriarty, "An advanced modeling system for optimization of wind farm layout and wind turbine sizing using a multi-level extended pattern search algorithm”, Energy 106, 802-814 (2016), doi:10.1016/j.energy.2015.12.033

[51] A. P. J. Stanley, J. Thomas, A. Ning, J. Annoni, K. Dykes, and P. Fleming, "Gradientbased optimization of wind farms with different turbine heights", $35^{\text {th }}$ Wind Energy Symposium, AIAA SciTech AIAA 2017-1619, 1-13 (2017), doi:10.2514/6.20171619.

[52] A. Vasel-Be-Hagh and C. L. Archer, "Wind farm hub height optimization", Applied Energy 195, 905-921 (2017), doi:10.1016/j.apenergy.2017.03.089

[53] A. P.J. Stanley, A. Ning, and K. Dykes, "Benefits of two turbine rotor diameters and hub heights in the same wind farm", $36^{\text {th }}$ Wind Energy Symposium, AIAA SciTech AIAA 2018-2016, 1-13 (2018), doi:10.2514/6.2018-2016.

[54] A. P. J. Stanley, A. Ning, and K. Dykes, "Optimization of turbine design in wind farms with multiple hub heights, using exact analytic gradients and structural constraints", Wind Energy 22, 605-619 (2019), doi:10.1002/we.2310

[55] A. P. J. Stanley and A. Ning, "Coupled wind turbine design and layout optimization with nonhomogeneous wind turbines", Wind Energy Science 4, 99-114 (2019), doi:10.5194/wes-4-99-2019 
[56] M. Abkar and F. Porté-Agel, "The effect of free-atmosphere stratification on boundary-layer flow and power output from very large wind farms", Energies 6, 2338-2361 (2013), doi:10.3390/en6052338

[57] J. Meyers and C. Meneveau, "Optimal turbine spacing in fully developed wind farm boundary layers”, Wind Energy 15, 305-317 (2012), doi:10.1002/we.469

[58] R. J. A. M. Stevens, B. Hobbs, A. Ramos, and C. Meneveau, "Combining economic and fluid dynamic models to determine the optimal spacing in very large wind farms", Wind Energy 20, 465-477 (2017), doi:10.1002/we.2016

[59] W. M. Organization, Guide to Meteorological Instruments and Methods of Observation, seventh edition (World Meteorological Organization (WMO), Geneva) (2008), ISBN: 978-92-63-10008-5

[60] P. B. T. Stehly and P. Duffy, "2019 cost of wind energy review", National Renewable Energy Laboratory, NREL/TP-5000-78471, Golden, CO (2019), www.nrel.gov.

[61] U.S. Energy Information Administration, "Cost and performance characteristics of new generating technologies, annual energy outlook 2021”, (2021), www.eia.gov.

[62] R. J. A. M. Stevens, J. Graham, and C. Meneveau, "A concurrent precursor inflow method for large eddy simulations and applications to finite length wind farms", Renewable Energy 68, 46-50 (2014), doi:10.1016/j.renene.2014.01.024

[63] J. D. Albertson, "Large eddy simulation of land-atmosphere interaction", Ph.D. thesis, University of California (1996).

[64] J. D. Albertson and M. B. Parlange, "Surface length-scales and shear stress: implications for land-atmosphere interaction over complex terrain”, Water Resour. Res. 35, 2121-2132 (1999), doi:10.1029/1999WR900094.

[65] M. Germano, U. Piomelli, P. Moin, and W. H. Cabot, "A dynamic subgrid-scale eddy viscosity model”, Phys. Fluids A 3, 1760-1765 (1991), doi:10.1063/1.857955

[66] R. J. A. M. Stevens and C. Meneveau, "Flow structure and turbulence in wind farms", Annu. Rev. Fluid Mech. 49, 311-339 (2017), doi:10.1146/annurev-fluid-010816060206

[67] E. Bou-Zeid, C. Meneveau, and M. B. Parlange, "A scale-dependent Lagrangian dynamic model for large eddy simulation of complex turbulent flows", Phys. Fluids 17, 025105 (2005), doi:10.1063/1.1839152 
[68] C.-H. Moeng, "A large-eddy simulation model for the study of planetary boundary-layer turbulence", J. Atmos. Sci. 41, 2052-2062 (1984), doi:10.1175/15200469(1984)041<2052:ALESMF>2.0.CO;2

[69] W. Munters, C. Meneveau, and J. Meyers, "Shifted periodic boundary conditions for simulations of wall-bounded turbulent flows", Phys. Fluids 28, 025112 (2016), doi:10.1063/1.4941912

[70] E. Branlard, Momentum Theory, In: Wind Turbine Aerodynamics and VorticityBased Methods. Research Topics in Wind Energy, volume 7 (Springer, Cham) (2017), doi:10.1007/978-3-319-55164-79.

[71] T. Burton, D. Sharpe, N. Jenkins, and E. Bossanyi, Wind Energy Handbook (John Wiley \& Sons, New York) (2001), doi:10.1002/9781119992714.

[72] J. N. Sørensen and W. Z. Shen, "Numerical modeling of wind turbine wakes", J. Fluids Eng. 124, 393 (2002), doi:10.1115/1.1471361

[73] B. Sanderse, S. P. van der Pijl, and B. Koren, "Review of computational fluid dynamics for wind turbine wake aerodynamics”, Wind Energy 14, 799-819 (2011), doi: $10.1002 /$ we. 458

[74] J. Meyers and C. Meneveau, "Large eddy simulations of large wind-turbine arrays in the atmospheric boundary layer", $48^{\text {th }}$ AIAA Aerospace Sciences Meeting Including the New Horizons Forum and Aerospace Exposition AIAA 2010-827 4 - 7 January 2010, Orlando, Florida AIAA 2010-827 (2010), doi:10.2514/6.2010-827

[75] A. Reinecke, D. Zhao, J. Cater, E. Goh, and N. Han, Wind Turbines and Aerodynamics Energy Harvesters (Academic Press) (2019), ISBN: 978-0-12-817135-6.

[76] R. J. A. M. Stevens and C. Meneveau, "Temporal structure of aggregate power fluctuations in large-eddy simulations of extended wind-farms", J. Renew. Sustain. Energy 6, 043102 (2014), doi:10.1063/1.4885114

[77] C. R. Shapiro, D. F. Gayme, and C. Meneveau, "Filtered actuator disks: Theory and application to wind turbine models in large eddy simulation", Wind Energy 1-7 (2019), doi:10.1002/we.2376

[78] R. J. A. M. Stevens, D. F. Gayme, and C. Meneveau, "Effects of turbine spacing on the power output of extended wind-farms", Wind Energy 19, 359-370 (2016), doi:10.1002/we.1835 
[79] Y.-T. Wu and F. Porté-Agel, "Large-eddy simulation of wind-turbine wakes: Evaluation of turbine parametrisations”, Boundary-Layer Meteorol. 138, 345-366 (2011), doi:10.1007/s10546-010-9569-x.

[80] Y.-T. Wu and F. Porté-Agel, "Atmospheric turbulence effects on wind-turbine wakes: An LES study”, Energies 5, 5340-5362 (2012), doi:10.3390/en5125340

[81] R. J. A. M. Stevens, L. A. Martínez-Tossas, and C. Meneveau, "Comparison of wind farm large eddy simulations using actuator disk and actuator line models with wind tunnel experiments”, Renewable Energy 116, 470-478 (2018), doi:10.1016/j.renene.2017.08.072.

[82] E. Barlas, S. Buckingham, and J. van Beeck, "Roughness effects on wind-turbine wake dynamics in a boundary-layer wind tunnel”, Boundary-Layer Meteorology 158, 27-42 (2015).

[83] M. Abkar and F. Porté-Agel, "Influence of atmospheric stability on wind-turbine wakes: A large-eddy simulation study”, Phys. Fluids 27, 035104 (2015).

[84] R. J. A. M. Stevens, D. F. Gayme, and C. Meneveau, "Using the coupled wake boundary layer model to evaluate the effect of turbulence intensity on wind farm performance”, J. Phys. Conf. Ser. 625, 012004 (2015), doi:10.1088/17426596/625/1/012004.

[85] R. B. Cal, J. Lebrón, L. Castillo, H. S. Kang, and C. Meneveau, "Experimental study of the horizontally averaged flow structure in a model wind-turbine array boundary layer”, J. Renew. Sustain. Energy 2, 013106 (2010), doi:10.1063/1.3289735

[86] C. S. Ferreira, H. A. Madsen, M. Barone, P. Deglaire, and I. Arduin, "Comparison of aerodynamic models for vertical axis wind turbines”, J. Phys.: Conf. Ser. 524, 012125 (2014), doi:10.1088/1742-6596/524/1/012125

[87] F. Ottermo and H. Bernhoff, "An upper size of vertical axis wind turbines", Wind Energy 17(10), 1623-1629 (2014), doi:doi.org/10.1002/we.1655.

[88] S. Shamsoddin and F. Porté-Agel, "Large eddy simulation of vertical axis wind turbine wakes”, Energies 7, 890-912 (2014), doi:10.3390/en7020890

[89] V. Mendoza, P. Bachant, C. S. Ferreira, and A. Goude, "Near-wake flow simulation of a vertical axis turbine using an actuator line model", Wind Energy 22(2), 171-188 (2019), doi:10.1002/we.2277. 
[90] A. Segalini and J.-Å. Dahlberg, "Blockage effects in wind farms", Wind Energy 23, 120-128 (2020), doi:10.1002/we.2413

[91] N. G. Nygaard, S. T. Steen, L. Poulsen, and J. G. Pedersen, "Modeling cluster wakes and wind farm blockage”, J. Phys. Conf. Ser. 1618, 062072 (2020), doi:10.1088/17426596/1618/6/062072

[92] D. Medici, S. Ivanell, J.-Å. Dahlberg, and P. H. Alfredsson, "The upstream flow of a wind turbine: blockage effect", Wind Energy 14, 691-697 (2011), doi:10.1002/we.451

[93] E. Simley, L. Y. Pao, P. Gebraad, and M. Churchfield, "Investigation of the impact of the upstream induction zone on LIDAR measurement accuracy for wind turbine control applications using large-eddy simulation”, J. Phys. Conf. Ser. 524, 012003 (2014), doi:10.1088/1742-6596/524/1/012003

[94] K. B. Howard and M. Guala, "Upwind preview to a horizontal axis wind turbine: a wind tunnel and field-scale study", Wind Energy 19, 1371-1389 (2016), doi:10.1002/we.1901

[95] E. Simley, N. Angelou, T. Mikkelsen, M. Sjöholm, J. Mann, and L. Y. Pao, "Characterization of wind velocities in the upstream induction zone of a wind turbine using scanning continuous-wave lidars", J. Renew. Sustain. Energy 8, 013301 (2016), doi:10.1063/1.4940025

[96] A. R. Meyer Forsting, N. Troldborg, J. P. M. Leon, A. Sathe, N. Angelou, and A. Vignaroli, "Validation of a CFD model with a synchronized triple-lidar system in the wind turbine induction zone", Wind Energy 20, 1481-1498 (2017), doi:110.1002/we.2103.

[97] A. R. Meyer Forsting, M. P. van der Laan, and N. Troldborg, "The induction zone/factor and sheared inflow: A linear connection?”, J. Phys. Conf. Ser. 1037, 072031 (2018), doi:10.1088/1742-6596/1037/7/072031

[98] S. McTavish, S. Rodrigue, D. Feszty, and F. Nitzsche, "An investigation of in-field blockage effects in closely spaced lateral wind farm configurations", Wind Energy 18, 1989-2011 (2015), doi:10.1002/we.1806.

[99] A. R. Meyer Forsting, N. Troldborg, and M. Gaunaa, "The flow upstream of a row of aligned wind turbine rotors and its effect on power production", Wind Energy 20, 63-77 (2017), doi:10.1002/we.1991 
[100] R. Ebenhoch, B. Muro, J. Dahlberg, P. Berkesten Hägglund, and A. Segalini, "A linearized numerical model of wind-farm flows”, Wind Energy 20, 859-875 (2017), doi:10.1002/we.2067

[101] J. Bleeg, M. Purcell, R. Ruisi, and E. Traiger, "Wind farm blockage and the consequences of neglecting its impact on energy production”, Energies 11, 1609 (2018), doi:10.3390/en11061609.

[102] E. Branlard and A. R. Meyer Forsting, "Assessing the blockage effect of wind turbines and wind farms using an analytical vortex model”, Wind Energy 23, 2068 2086 (2020), doi:10.1002/we.2546

[103] E. Branlard, E. Quon, A. R. Meyer Forsting, J. King, and P. Moriarty, "Wind farm blockage effects: comparison of different engineering models", J. Phys. Conf. Ser. 1618, 062054 (2020).

[104] J. Bleeg, "A graph neural network surrogate model for the prediction of turbine interaction loss", J. Phys. Conf. Ser. 1618, 062054 (2020), doi:10.1088/17426596/1618/6/062054

[105] A. Segalini and J.-Å. Dahlberg, "Global blockage effects in wind farms", J. Phys. Conf. Ser. 1256, 012021 (2019), doi:10.1088/1742-6596/1256/1/012021

[106] A. Sebastiani, F. Castellani, G. Crasto, and A. Segalini, "Data analysis and simulation of the Lillgrund wind farm”, Wind Energy 24 (2020), doi:10.1002/we.2594

[107] A. Jimenez, A. Crespo, E. Migoya, and J. Garcia, "Advances in large-eddy simulation of a wind turbine wake”, J. Phys. Conf. Ser. 75, 012041 (2007), doi:10.1088/1742$6596 / 75 / 1 / 012041$

[108] N. Troldborg, J. N. Sørensen, and R. Mikkelsen, "Numerical simulations of wake characteristics of a wind turbine in uniform inflow”, Wind Energy 13, 86-99 (2010), doi:10.1002/we.345

[109] J. N. Sørensen, "Aerodynamic aspects of wind energy conversion", Annu. Rev. Fluid Mech. 43, 427-448 (2011), doi:10.1146/annurev-fluid-122109-160801.

[110] M. P. van der Laan, N. N. Sørensen, P. E. Réthoré, M. C. Kelly, and J. Mann, "Efficient turbulence modeling for CFD wake simulations", Ph.D. thesis, DTU Wind Energy (2014).

[111] P. E. Réthoré, M. P. van der Laan, N. Troldborg, F. Zahle, and N. N. Sørensen, "Verification and validation of an actuator disc model", Wind Energy 17, 919-937 (2014), doi:10.1002/we.1607. 
[112] N. Troldborg and A. R. Meyer Forsting, "A simple model of the wind turbine induction zone derived from numerical simulations", Wind Energy 20, 2011-2020 (2017), doi:10.1002/we.2137.

[113] S. McTavish, D. Feszty, and F. Nitzsche, "A study of the performance benefits of closely-spaced lateral wind farm configurations", Renewable Energy 59, 128-135 (2013), doi:110.1016/j.renene.2013.03.032

[114] J. M. I. Strickland and R. J. A. M. Stevens, "Effect of thrust coefficient on the flow blockage effects in closely-spaced spanwise-infinite turbine arrays", J. Phys. Conf. Ser. 1618, 062069 (2020), doi:10.1088/1742-6596/1618/6/062069

[115] K. L. Wu and F. Porté-Agel, "Flow adjustment inside and around large finite-size wind farms”, Energies 10, 2164 (2017), doi:10.3390/en10122164.

[116] D. Allaerts and J. Meyers, "Boundary-layer development and gravity waves in conventionally neutral wind farms", J. Fluid Mech. 814, 95-130 (2017), doi:10.1017/jfm.2017.11

[117] D. Allaerts and J. Meyers, "Gravity waves and wind-farm efficiency in neutral and stable conditions”, Boundary-Layer Meteorol. 166, 269 (2018), doi:10.1007/s10546017-0307-5.

[118] D. Allaerts and J. Meyers, "Sensitivity and feedback of wind-farm-induced gravity waves”, J. Fluid Mech. 862, 990-1028 (2019), doi:10.1017/jfm.2018.969.

[119] A. R. Meyer Forsting and N. Troldborg, "The effect of blockage on power production for laterally aligned wind turbines", J. Phys. Conf. Ser. 625, 012029 (2015), doi:10.1088/1742-6596/625/1/012029.

[120] F. Porté-Agel, Y.-T. Wu, and C. H. Chen, "A numerical study of the effects of wind direction on turbine wakes and power losses in a large wind farm", Energies 6, 5297-5313 (2013), doi:10.3390/en6105297.

[121] R. J. Barthelmie, G. Larsen, S. Pryor, H. Jørgensen, H. Bergström, W. Schlez, K. Rados, B. Lange, P. Vølund, S. Neckelmann, S. Mogensen, G. Schepers, T. Hegberg, L. Folkerts, and M. Magnusson, "ENDOW (Efficient Development of Offshore Wind farms): Modelling wake and boundary layer interactions", Wind Energy 7, 225-245 (2004), doi:10.1002/we.121

[122] D. Medici and P. H. Alfredsson, "Measurement on a wind turbine wake: 3D effects and bluff body vortex shedding”, Wind Energy 9, 219-236 (2006), doi:10.1002/we.156 
[123] S. McTavish, D. Feszty, and F. Nitzsche, "An experimental and computational assessment of blockage effects on wind turbine wake development", Wind Energy 17, 1515-1529 (2014), doi:10.1002/we.1648.

[124] R. B. Stull, An Introduction to Boundary Layer Meteorology (Kluwer Academic publishers, Boston) (1988), doi:10.1007/978-94-009-3027-8

[125] S. N. Gadde and R. J. A. M. Stevens, "Interaction between low-level jets and wind farms in a stable atmospheric boundary layer", Phys. Rev. Fluids 6, 014603 (2021), doi:10.1103/PhysRevFluids.6.014603

[126] B. Kosović and J. A. Curry, "A large eddy simulation study of a quasi-steady stably stratified atmospheric boundary layer”, J. Atmos. Sci. 57, 1052-1068 (2000), doi:10.1175/1520-0469(2000)057<1052:ALESSO>2.0.CO;2.

[127] R. Stoll and F. Porté-Agel, "Large-eddy simulation of the stable atmospheric boundary layer using dynamic models with different averaging schemes”, Boundary-Layer Meteorol. 126, 1-28 (2008), doi:10.1007/s10546-007-9207-4

[128] A. Sescu and C. Meneveau, "A control algorithm for statistically stationary largeeddy simulations of thermally stratified boundary layers”, Q. J. R. Meteorol. Soc. 140, 2017-2022 (2014), doi:10.1002/qj.2266

[129] A. Sescu and C. Meneveau, "Large-eddy simulation and single-column modeling of thermally stratified wind-turbine arrays for fully developed, stationary atmospheric conditions”, J. Atmospheric Ocean. Technol. 32, 1144-1162 (2015), doi:10.1175/JTECH-D-14-00068.1

[130] J. A. G. R. Stoll, S. T. Salesky, W. Anderson, and M. Calaf, "Large-eddy simulation of the atmospheric boundary layers”, Boundary-Layer Meteorology 177, 541-581 (2020), doi:10.1007/s10546-020-00556-3.

[131] M. Abkar, A. Sharifi, and F. Porté-Agel, "Large-eddy simulation of the diurnal variation of wake flows in a finite-size wind farm”, J. Phys. Conf. Ser. 625, 012031 (2015), doi:10.1088/1742-6596/625/1/012031

[132] S. N. Gadde, A. Stieren, and R. J.A. M. Stevens, "Large-eddy simulations of stratified atmospheric boundary layers: Comparison of different subgrid models", BoundaryLayer Meteorol. 178, 363-382 (2021), doi:10.1007/s10546-020-00570-5.

[133] M. S. Gomez, J. K. Lundquist, J. D. Mirocha, R. S. Arthur, and D. Munoz-Esparza, "Quantifying wind plant blockage under stable atmospheric conditions", Submitted to Wind Energy Science (2021). 
[134] S. N. Gadde and R. J. A. M. Stevens, "Effect of Coriolis force on a wind farm wake", J. Phys. Conf. Ser. 1256, 012026 (2019), doi:10.1088/1742-6596/1256/1/012026

[135] S. N. Gadde, "Large eddy simulations of stratified atmospheric boundary layers and wind farms", Ph.D. thesis, University of Twente (2021), doi:10.3990/1.9789036551809.

[136] Á. Jiménez, A. Crespo, and E. Migoya, "Application of a LES technique to characterize the wake deflection of a wind turbine in yaw", Wind Energy 13, 559-572 (2010), doi:10.1002/we.380

[137] M. Calaf, M. B. Parlange, and C. Meneveau, "Large eddy simulation study of scalar transport in fully developed wind-turbine array boundary layers”, Phys. Fluids 23, 126603 (2011), doi:10.1063/1.3663376

[138] R. Verstappen, "When does eddy viscosity damp subfilter scales sufficiently?", J. Sci. Comp. 49, 94 (2011), doi:10.1007/s10915-011-9504-4

[139] M. Abkar, A. Sharifi, and F. Porté-Agel, "Wake flow in a wind farm during a diurnal cycle”, J. Turb. 17, 420-441 (2016), doi:10.1080/14685248.2015.1127379

[140] M. Abkar and P. Moin, "Large eddy simulation of thermally stratified atmospheric boundary layer flow using a minimum dissipation model", Boundary-Layer Meteorol. 165, 405-419 (2017), doi:10.1007/s10546-017-0288-4

[141] C. Canuto, M. Y. Hussaini, A. Quarteroni, and T. A. Zang, Spectral Methods in Fluid Dynamics (Springer, Berlin) (1988), doi:10.1007/978-3-642-84108-8

[142] J. H. Ferziger and M. Perić, Computational methods for fluid dynamics (Springer) (2002), doi:10.1007/978-3-642-56026-2

[143] W. Brutsaert, Evaporation into the atmosphere: theory, history and applications, volume 1 (1982), doi:10.1007/978-94-017-1497-6

[144] V. Kumar, G. Svensson, A. A. M. Holtslag, C. Meneveau, and M. B. Parlange, "Impact of surface flux formulations and geostrophic forcing on large-eddy simulations of diurnal atmospheric boundary layer flow", J. Appl. Meteorol. Climatol. 49, 14961516 (2010), doi:10.1175/2010JAMC2145.1

[145] D. G. Dommermuth and D. K. P. Yue, "A high-order spectral method for the study of nonlinear gravity waves", J. Fluid Mech. 184, 267-288 (1987), doi:10.1017/S002211208700288X 
[146] L. Lanzilao and J. Meyers, "Set-point optimization in wind farms to mitigate effects of flow blockage induced by atmospheric gravity waves", Wind Energ. Sci. 6, 247-271 (2021), doi:10.5194/wes-6-247-2021

[147] R. J. Beare, M. K. Macvean, A. A. M. Holtslag, J. Cuxart, I. Esau, J.-C. Golaz, M. A. Jimenez, M. Khairoutdinov, B. Kosović, D. Lewellen, T. S. Lund, J. K. Lundquist, A. Mccabe, A. F. Moene, Y. Noh, S. Raasch, and P. Sullivan, "An intercomparison of large eddy simulations of the stable boundary layer", Boundary-Layer Meteorol. 118, 247-272 (2006), doi:10.1007/s10546-004-2820-6

[148] A. K. Blackadar, "Boundary layer wind maxima and their significance for the growth of nocturnal inversions”, Bull. Am. Meteorol. Soc. 38, 283-290 (1957), doi:10.1175/1520-0477-38.5.283

[149] S. Gadde, L. Liu, and R. Stevens, "Effect of low-level jet height on wind farm performance”, Journal of Renewable and Sustainable Energy 13, 0013305 (2021), doi:10.1063/5.0026232

[150] M. Magnusson and A. S. Smedman, "Air flow behind wind turbine", J. Wind Eng. Ind. Aerodyn. 80, 169-189 (1999), doi:10.1016/S0167-6105(98)00126-3

[151] S. Gadde, L. Liu, and R. Stevens, "Effect of low-level jet on turbine aerodynamic blade loading using large-eddy simulations”, J. Phys. Conf. Ser. 1934, 012001 (2021), doi:10.1088/1742-6596/1934/1/012001

[152] S. S. Zilitinkevich, T. Elperin, N. Kleeorin, I. Rogachevskii, I. Esau, T. Mauritsen, and M. W. Miles, "Turbulence energetics in stably stratified geophysical flows: Strong and weak mixing regimes”, Q. J. R. Meteorol. Soc. 134, 793-799 (2008), doi:10.1002/qj.264 


\section{Acknowledgements}

They say a calm sea never made a good sailor. Well, for my sake, I hope it holds true. I have always loved science and learning. Yet, growing up how I did, a lot of people already wrote me off from the beginning. Later, when I worked a minimum-wage job to save up and focus on my mental health, people kept telling me I would never go back to university. And when I did, I still had some people telling me I wouldn't make it. Well, here I am. It hasn't been easy, especially finishing a $\mathrm{PhD}$ in another country during a global pandemic, but I made it. I have had so many beautiful experiences along the way and I want to take this space to thank the people who supported me and helped me - not only during these four years. I wouldn't be here without you, truly.

First of all I would like to thank Detlef Lohse and Richard Stevens. Quite literally, I would not be here without you because you selected me for this project.

Detlef, who knew in my earlier years, in a tiny town, when I was citing you in a Sonoluminescence report, that I would end up here. It has been a privilege to be a part of your group. Thank you not only for your time, but also for your empathy and support throughout my $\mathrm{PhD}$.

Richard, thank you particularly for selecting me for such a cool project. I was one of your first PhD students and growing and working together over these years has been a privilege. I have greatly appreciated your time, your insight, and your listening to my grievances. Also, the bonding you foster in the small wind farm group that you created has helped to make this a pleasant experience. Thank you for the kind words and constructive criticism that has helped me grow into a better scientist. And, thank you for always reminding me that things will never be perfect.

I would also like to express my gratitude to my committee Roberto Verzicco, Wilko Rohlfs, Carlos Simão Ferreira and Anna von der Heydt, for taking the time to read and evaluate my thesis. Anna, the fact that you taught my first GFD course makes your presence especially nice. And, thank you to my chair person Srirang Manohar for your comforting words in preparation for the defense. 


\section{My Paranymphs}

Anja, I know I've told you times before how glad I am you're here, but here it is in writing: Thank you for being by my side today. You've been by my side quite literally in the office, but you've also been there for most of the fun (and not-so-fun) moments throughout my PhD. Having such a great friend working on what you do is truly such a blessing. I cannot imagine my PhD without you. Of course, thanks for all of your help and expert opinions, especially in the making of this thesis. However, I'm also so grateful for all the chats and laughs that made me feel sane. Last, I gotta say it: thanks for being older than me.

Ricardo, my fellow North American. Once I insignificantly texted you about an event and you replied "you can count on me". I thought in that moment: I've always been able to. You've always been there for me and I've told you so many times how grateful I am for you. As internationals so far from family, and academia that constantly flows, I'm so glad I got to keep you for four years. I've never met such a kind and hard-working person that doubts their kindness and hard work like you do. Yet, somehow with all this, you still manage to be there for your friends. Thank you for all of the support and all of the adventures. We'll never get to do all the things we said we would do, but I think we did a great job trying.

\section{The other wind farmers}

Srinidhi Nagarada Gadde, thanks for being patient when I was calling you Srin (and in general). Wow. You've been here since the beginning and there's so much I couldn't have done without you. Thank you for everything, including all the fun we have had together. I feel like we have both grown so much in four years, with rough patches only making us stronger. You have been a great friend and it's been an honor to work with you. You're so gifted and I'm sure you have great things ahead of you, especially with Mitali by your side.

Luoqin, you work so hard and are such a friendly and helpful person. You have always made me feel comfortable asking you questions in and out of science. You may be leaving, but I'm grateful you stayed as long as you did. Thank you as well for introducing me to your beautiful family: Danqi and Peppa are absolute delights. I'm so happy for you all in your next chapter.

Though our group is small, yet there are some people I only overlapped with briefly. Mengqi, thank you for introducing me to vertical staggering and thank you for your desk :p. Ariane, we did not get to do much during the pandemic, but it was still nice to have another person in the group. And finally, Jens, welcome to the group. You just got here, and yet you've already been a big help. I wish you so much success and I hope that you somehow manage to have even more good times in this group than I did. 


\section{'Pof'fertjes}

I'm so grateful to have worked in this ever-changing, talented group. People come and go, but so many of you have touched me in different ways and helped me along my journey, whether it be scientific input, encouragement, or allowing a safe place for me to complain - as I like to do. The memories I have made here, I will cherish for a lifetime and the following tributes will simply not do any of you the justice you deserve.

To start, I would like to acknowledge the OG RUN group that had little to do with running in the end. In fact, as opposed to running, many of you helped me face problems. First, Myrthe, I don't think I would have sported half as much without your nudge. You also helped give me confidence in ways. Thank you for bringing so much energy and fun. Liz, thank you for always making me so comfortable, yet inspiring me to make people uncomfortable: I am so truly grateful for women like you who are not afraid to use their voices. It's people like you who actually change things. Carola, thank you for the times you were there for me and your expert opinions. And, maybe this is not obvious to you, but you inspire me so much with your strength. Also, thank you for all the times you reminded me I can stop digging at any time. Maaike, thank you for having been such a special friend to me. You're so kind, sensitive, and understanding. You have a warmth that resonates and an enthusiasm that fires me up. You were one of my first friends at pof and you have been ever since. So, we have way too many memories to list, which I am of course grateful for, but also you have helped me so much along this journey. The best way to sum it up is to say thank you for having been a part of my family here. And to all of you, I hope we run into each other again someday.

I have had quite a few office-mates over the years. And to all of you, I want to say: you're welcome. I'm joking, of course. I really don't know how any of you got anything done with me around. To the first office-mates of ME248: Borge, thank you for reminding me there are more important things in life sometimes. Anaïs, without you and a miscommunication, I would never have known that I can walk my Kat. Yaxing, your friendship has been so special to me because we are so different, yet we understand each other in many ways. I appreciate the comfort, the openness, and the laughs. And of course, thank you for helping me analyze my emails. Since you all, I have had new office-mates. Steven, you were always great to talk to and thank you for ignoring how disruptive the rest of us could be. Edgar, I think you tried to win me over with bonus chocolate, and of course it works. Also, thanks for checking in during the pandemic. Morgan, you're the newest addition, yet you probably know me so well with all the conversations I have dumped on you. It may have taken 6 months to find out what you do, but it's because we were so enthusiastically chatting about so many things. Thanks for being a good friend, especially coming out of these rough times. Much like your baking, you are a delight. 
To the neighboring offices, especially Bernardo, Daniël, Diana, and Yanshen, thank you for your patience. I could hear you sometimes, which meant you had to hear me always. Yanshen, you practically lived in that office. You are such an interesting character, which I knew that moment you offered me some smuggled-in soda at the theater, but I had also smuggled in my own. Thanks for the laughs and also the comforts you gave me. Diana, thank you for sharing mutual American immigrant problems and potential solutions. Daniël, thank you for enduring my many pop-ins and for always being so friendly. Bernardo, thanks for giving me confidence that the fun-times of the group are in good hands in the next generation. On the other side of our office, with a thicker wall, I made sure to drop by constantly so you could hear my voice. Utkarsh, thanks for being a safe place to dump the dark stuff and for the dank memes. It's great to have friends who understand the lessons that only the Trailer Park Boys can teach. Martin A., I appreciate your style of humor so much and I have missed your presence in the end. You don't find too many people willing to jump into a ball pit with you at 3am. Speaking of balls, Jelle, thanks for the stupid smiles I couldn't wipe off my face if I tried. Also, I'm sorry that you suck at Yu-Gi-Oh cards and that you feel the need to make excuses for that.

This group is full of such cool and diverse people that I have had the pleasure of meeting. Unfortunately (for you), I am apparently very social and this paragraph will be long. If you're still reading this, I am surprised you didn't just skim for your name - I even put it in bold for you. Ali, thank you for being party MVP. Çayan, you just got here but thanks for being such a friendly neighbor. It makes me feel more at home. Charu, thanks for being a downer sometimes. Our conversations are so refreshing. Duco, you also just got here, but maybe that is a good thing, because I would never get anything done with the amount that you and I can talk. Farz(h)an, I'm glad that we have connected over the last while. You're a great person to complain to and thank you for setting the standard for volleyball so low. I'm joking. You say that I make it not-funny when I say that I am joking. And well, I don't need to remind you you're an expert in the that because the mirror does everyday. I'm joking, jeeez. Marvin, I wouldn't have joined Harambee if not for you and it changed my life during the pandemic. But in general, thank you inspiring me to do new things, for encouraging me to dance, even if I can't, and to sing, even if I probably shouldn't. And, thank you for all-round just being a great friend. Mathieu, thank you for helping me and Luca, but for also just being a great colleague to talk to especially about pandemic struggles and cats. I don't know how you and Sandra pull off what you do, but it inspires me. Michiel, thank you for letting me razz you and all the lunch walk chats we had over the years. Pierre, thanks for being 'mean' when I asked for opinions. I really mean that. Robert (bobby), thank you for your help. But in general, I feel bad for anyone who falls victim to you and I conversing. Your enthusiasm is infectious. I don't understand how you turned out to be such a happy-spirited guy raised on the creepy German lessons you share. That being said, I will never not eat my soup. Vanshu, thank you for being so 
understanding and supporting me against Robert's controversial ice coffee opinions. W. Pim W., frat boy, you are a surprising character and I'm so grateful for a neighbor like you. Thanks for looking out. Youssef, you kept running away, but I'm grateful for the times you were here because you are a good soul and we have had lots of fun. Yoan, thanks for being such a kind and friendly face. Now, thanks to the guys who kept inviting me to play cricket while definitely going easy on me. Minkush, you are such a delight. You are so kind and thoughtful to everyone. Also, thank you for letting me take your turbulence notes forever ago when I started the $\mathrm{PhD}$ and immediately left. Srinath, thank you for always welcoming me into your home and for the fun times we have shared. Yogesh, it's been nice knowing when I'm at the office at $11 \mathrm{pm}$ that there is another burdened soul wandering the halls, willing to share their coffee milk. Vatsal, thank you for your help but also your comfort, especially during the harder times. There are so many other people in pof that I share memories with or who have provided well-needed distractions: Alexandros, Ambre, Ben, Binglin, Chris, Dawid, Ellis, Giulia, Hadi, Hari, Jochem, Lijun, Martin E., Nathan, Nayoung, Saeed, Sarah, Timothy, Udo, Vincent, Xiaolai, and Yibo - thank you all for making this experience such a memorable one.

I would be remiss not to mention that the professors and technical staff at pof have also been very friendly and helpful throughout the years. I would like to give special mention to Baas, as a numerics person, thank you for dealing with a bulk of my issues and showing me so many things. Dennis vG., to put it simply, thank you for being an ally. Gert-Wim, I may not have needed you in the same way that most people in pof do, but I still appreciated your kind words and of course the eggs. The chats we had on the outing had a large impact on me. And finally, Joanita, we all appreciate you because you keep everything going. But, I want to thank you also for being so much more. When I am so far from home and family, warm and caring people like you have helped to ease that pain.

Well, if you think that list was long, I am sorry, but I still need to express my gratitude to several pof people who have come and gone. Biljana, one of my first pof friends, thank you for your help in the bouldering hall and for being there when Ruben ridiculously said there are no feminine women in engineering. Ruben, you were ridiculous sometimes, but very welcoming in other ways. You were one of the first people to make me feel welcome in the group and always made time for a chat and a Dutch sandwich. Vamsi, you were also so welcoming when I first arrived and really brought me into the group. Pablo, thank you for bestowing your humor on pof. Peter D., I'm glad that you stuck around after you finished because you bring such an energy to the group that is unlike anyone else. Never doubt your worth. Kirsten, I admired your boldness, and I think the night-dip you initiated is a good example of that. Pallav, I didn't get to see you much, being locked away downstairs, but you were always enthusiastic in group activities, helping to film movies and decorate pof for Christmas together. CS, with you I think of betrayal, baking, and bows. And of course, Cindy's amazing food and your professional presentation feedback. 
I used to go to lunch, because an hour is well-wasted on the conversations this group came up with. Thank you, Álvaro, Alex, Dennis B., Ivan, José, Mazi, Misha, and Rodrigo. I appreciated the authenticity, and the diversity of opinions, but more than anything the laughs. Alex, thank you for helping me, but also for making me feel like I am not crazy. Inside and outside of pof, Álvaro, you never failed to make me smile. Thank you for always making me feel included and for bringing an unmatched joy to everything. Dennis B., I would never have conquered a $13 \mathrm{~km}$ obstacle run without you, let alone even dare to think about trying it. Ivan, you warned me that you were a special bunch and you were right. That being said, I'm grateful our paths crossed. Also, $X$ gon' give it to $y a$ is the best hype-up song. José, thank you for one of the best work days I have ever had, not at work but on the Geertrui. Thank you for all the times you let me vent and made me laugh. Mazi, you have such as ease I admire and thank you for the times you made me feel included. Misha, thank you for being such a good listener - I know it couldn't have been easy sometimes haha. Rodrigo, hey Mexico, thank you for being an empathetic ear to complain to, even now. I didn't see it coming, but I should've, considering someone as sweet as Carola was so into you. To all the oldies, it has been a privilege to have shared even a fraction of this time with you.

\section{Outside Pof}

First, I would like to express my gratitude to Utrecht University and the IMAU group for the scholarship that allowed to start my studies in the Netherlands and the amazing program they provided for studying Climate through a physics lens.

I took a big leap moving to another country for my MSc and I am so grateful to those of you who made the transition easier. Vera, my official assigned Dutch buddy. Who knew, you would be helping me with so many Dutch things beyond the first weeks. But more importantly, you've been such a good friend all these years. You are very kind, fun, and a great listener. You have helped to make me feel safe and welcome when I'm so far from home. I still remember my first Sinterklaas, the time you brought chocolate to my house on a really sad day, and how you played my mom at my MSc graduation. We have way too many memories to list, but luckily we have all the snaps. Robin, thank you for being one of my first and most cherished Dutch friends. I was so happy to have a classmate like you and so heartbroken when you left. Somehow in our 20's, we were still getting in trouble for giggling in class. Those 'flat bottom approximations', though. You are so fun and so you. We have many memories and adventures that I cherish, like hiking in the mud singing Now that we're men and going to Manchester - twice. You turned an awkward moment for me into me my most beloved Dutch souvenir that I will keep forever and thank you also for playing my dad at my MSc graduation, mustache included.

Outside of the classroom and office, many people have kept me going over the years. I 
will start with my two best friends. Luca, I felt like stealing your well-put acknowledgement and just writing it back to you but I don't want you to feel that I feel like... Allora, thank you for being such family and comfort these last years (also from Kat). I am so blessed to have shared my home and this chapter with such a hilarious, beautiful and empathetic soul that resonates so deeply with mine. You have been a light in the dark, a laugh in despair, and I hope the world never dulls your sparkle. Excuuuuuuuuse me. Luiza, you are so unique and special, as simple as that. When we part ways, there will be a spot that can never be replaced. You have been such a great friend these years, and every time we are together my heart becomes fuller. Thank you for the laughter, the equinoxes, the slumber parties, the singing, the dancing, and just for being you. You are a beautiful person and I hope this world never hardens you like it has tried to. I wish there was an appropriate nerd quote that I could remember, but what comes to mind is from Winnie-the-Pooh: "You are braver than you believe, stronger than you seem, smarter than you think, and loved more than you know."

I have met so many beautiful people throughout my $\mathrm{PhD}$ that I cannot mention them all. But, to name a few: nanoFe, I tell you, so you know, but I still think you cannot imagine how grateful I am that you came into my life when and how you did. You will always have welcome a place in my heart and my home. You are so strong (I don't mean the working out) and yet delightfully soft. Thank you for all your support, and I hope you crush all of your goals. Você tem. Wenyi, thank you for inspiring me by always being your authentic self. Hossein, thank you, most importantly, for reminding me at $3 \mathrm{am}$, that it's supposed to feel impossible sometimes. Thank you for helping to take care of me and giving me the space to be myself.

I also want to express my gratitude to the good partners I have had in my life, Ernest, Truls and Koen. Each of you got a different version of me but you loved me unconditionally, were my biggest supporters, and helped me grow into the person I am today.

\section{Canada}

My success here is based on the foundation I received in my home province of Newfoundland. Before I left my BSc, I gave a letter to each of the professors who made such a profound difference in my life, and the sentiments still hold true. As I have progressed, I have just become increasingly grateful for all of your guidance, time, and continued support. I would not have pursued research without you.

Outside of Grenfell, but during my time there, I had the privilege of completing multiple research projects with multiple supervisors. I am grateful to each of you to have had the opportunity to do so as you helped to spark my passion for research. I would like to specifically mention Dr. Manuela Venturi who greatly impacted my life in science. I hid so much before I met you, a brilliant physicist with fuzzy pink stationary. You are 
an inspiration and thank you for your guidance at CERN. I made quite a few friends along my path and I am grateful to them all. I would like to express specific gratitude to Dr. Chelsea and soon-to-be Dr. Megan, two incredible physicists. Thank you for your friendship and help despite the time and space.

Even earlier, it was the support of many teachers who cultivated my love for education. To highlight some, Ms. Hynes, thank you for not giving up on me and for truly seeing me. Thank you, along with Ms. Crane and later Dr. Ganz, for giving me a great introduction to writing with techniques that I still use today. More importantly, thank you for giving me the confidence to do so. Also, thank you to Mr. Sanders, for making physics seem like the funnest thing you can imagine. To the rest of my teachers who saw me shine, helped me grow, and overlooked my failings, I can never thank you enough.

Outside of school, I am very grateful for the opportunities I was given thanks to The Royal Canadian Sea Cadets. Their programs gave me a taste for adventure, the interpersonal skills which are so useful to this day, and a passion for teaching. More importantly, here I truly learned to fail, which changed me for the better.

I have come as far as I have, in no small part, due to the family and friends I have had throughout my life that have loved me in failings and in successes. To start at the beginning, I had some pretty great friends growing up Kayla, Brittany, Shauna, and Harley, who welcomed my urge to dump information on people and never made me feel bad for being a brainer. Speaking of nerds: Charles, we met at a math competition over a decade ago and have been friends ever since. Your unapologetic weirdness even before university has always inspired me and you never fail to make me laugh no matter how much time passes. You are truly yourself. Thank you for your help, but more importantly for always being there. PS, wow look how old we are. Sarah, my longest friend. I cherish all of our memories, and I look forward to seeing you and Brandon every time I come home. You help make home feel like home which has kept me grounded when I struggle, so far away. I am so blessed to have had someone all these years who understands where I come from and is there no matter where I go. Thank you for the slumber parties at 8 and 28. I'll always be there to catch you when you fall. Not.

Amy, God bless Sarah for bringing you into my life. Thank you for every bit of help you ever gave me, including during my $\mathrm{PhD}$, like letting me use your office and when I got stuck, I wouldn't have gotten back the second time without you and Taylor. But more importantly, thank you for being a safe port. Places like floorbed always ground me when things are overwhelming. Jena, thank you for hearing me about the hard things. Your company is such a safe and cozy space. Mike, you have been a much-needed constant in my variable life. Thank you for the love and support you have given me all these years. Sometimes I felt like you put me on a pedestal, but at the same time it is such a blessing to have a friend who thinks you're greater than you are, especially when things are hard. You take such good care of your friends, and I am grateful to have been one of them. And last, 
Nicole, my Newfie Dad. We met during my introvert phase so I'm so glad that Grenfell forced you to do a Physics course and that you were a librarian or else we may not be here today. I appreciate that we can talk excitedly for hours or quietly do math. You're the only friend to visit me in Twente and it's been so refreshing to have a friend who understands two of my worlds. Especially when I was stuck during the pandemic, I made it through partially because of you, so thank you. Most importantly, thank you for reminding me that with good friends, I can be happy anywhere: Flavor of love and McDonald's in Paris seem to be a pretty good example.

\section{Family}

Is it strange to start with my cat? I guess not when she is literally on the cover. Kat, you can't read, so I will just give you extra wet food tomorrow or something. My cat has been a life-saver, pestering me ahem $^{*}$ I mean reminding me, that there is always time for snuggles and rest. Thank you for making my house in the Netherlands feel like a home.

Back in Canada, although we are not super close, I have many Aunts and Uncles who have supported me in different ways and I am very grateful. I would like to give a special mention to my Late Great Aunt Murt, who was so excited for me going to CERN and who actually read part of my Master's thesis. I would also like to acknowledge my Great Aunt Ruby. Thank you for telling me how beautiful I am and suggesting that I quit school and go into modeling. I got it half right.

Nan Strickland, thank you for adamantly telling her that I am good at school and I should stay. But seriously, thank you, especially for being there and being the support I needed during my depression and for always supporting my dreams. You are so wise and you give all of yourself. I am forever grateful for your ability to make everyone feel loved and how you express this often on my facebook posts. I love you and miss you terribly. Pop, thanks for always giving me the tightest hugs and for helping to give me an appreciation for nature that I still have today. I know you're worried about me not having a husband, but at least I have this thesis. Nan Stimatze, or Nanny far far away, you are so spirited and I love you. I am grateful to have had you as an inspiration of a strong woman who worked hard and made a life for herself in another country. And yet, ironically, thank you for being one of the ones who stuck around. I cherish every dinner, drive, and gossip session. I may be good at math, but I will never be able to count cards and dice at your caliber, so thanks for doing it for me every time.

Lori, thank you for being such a good friend over all these years. Thank you for every hug and every pick-me-up. When I was a kid freaking out about my future, you told me which high-school courses (sciences) I would need to do. I wrote them down, and I guess I accidentally stuck to the plan a bit. Even at 28, thanks for cutting up my apples and making me the best hot chocolate, foldy. Me Ol' Fadder, when you were there, you 
quizzed me and pushed me with questions constantly and I think a great deal of that made me the scientist I am today asking 'Why?'. You find so much joy in sharing information, which is definitely something that I find in me, and also in Jaron. Thank you for all the sharing and thank you for giving me Jeffrey's math even though it was grades ahead of me. You always made me feel that I have a voice that should be heard and that I could do anything and be anything. Love, weenie. Jeffrey, yer me brudder, and so I don't know who I would be without you having been there. I am glad to have someone who has felt and understands things that only we share. But most importantly, thank you for your son.

Jaron, my little jeezler, thank you for grounding me and for always reminding me that Saturday mornings are for playing and cartoons. You are smart, patient, and kind. Thank you for the adventures, the stories, and the hugs. But even more, thank you for thinking I'm cooler and younger than I will ever be again. It makes me so happy that you are as proud of me catching a shark in animal crossing as you are about my science. At my lowest, you lift me up. When I am down, Nanny reminds me how much you love me and I look back on all the years we've spent together and all the adventures we have ahead.

Me Ol' Mudder, where do I start when everything started with you? Some of my favorite parts of me come from you. Thank you for being my home. Thank you for every struggle and every snuggle. Because of you I have had an example such a strong woman since I was born. I appreciate so much, that after everything you have faced, you have given everything you could to us. Thank you, not only for always making sure I had what I needed, but for reading to me every night, making sure I did my homework, and teaching me the importance of education. Thank you for always giving me the space to be me and make my own choices. This thesis itself means so little because you have loved me unconditionally. Yet, it means so much because you always supported all of my ever-changing dreams from being a poet, journalist, photographer, and finally a scientist.

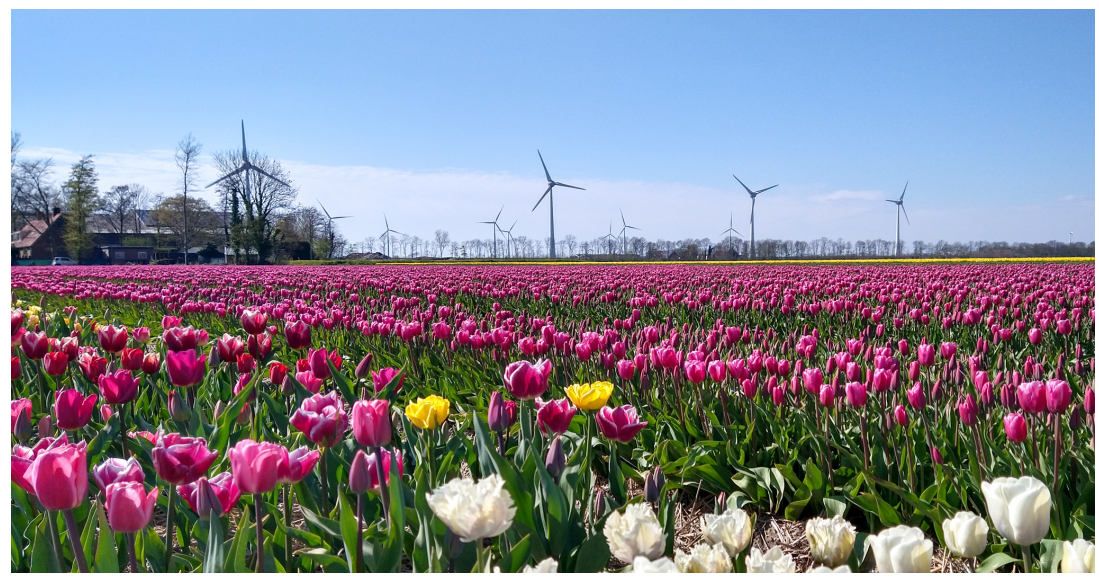




\section{About the author}

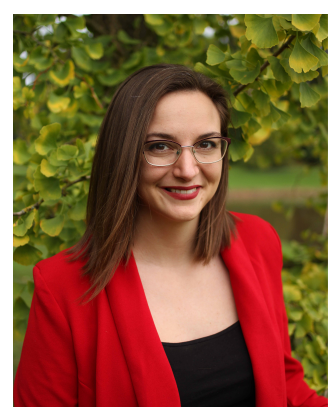

Jessica Marjorie Irene Strickland was born in Corner Brook, Newfoundland, on July $10^{\text {th }}, 1992$. It is nearly impossible to grow up in such a place without developing a fascination for climate and a deep appreciation of the environment. Her interest in physics and teaching was sparked during sailing courses given by the Royal Canadian Sea Cadets, for whom she later worked for as a sail instructor for many years. In 2015, she obtained a Bachelor's degree in Physics with a minor in Geography from Memorial University of Newfoundland and was awarded a medal of excellence for physics. Throughout this degree, she gained experience working as a supplemental instructor, lab assistant, observatory assistant, and barista (if you can call slinging coffee for Tim Hortons that). She was privileged to accept multiple Undergraduate Student Research Awards from NSERC, specializing in high energy particle physics. Her first research project involved investigating theoretical dark matter force carriers under the supervision of Dr. Aleksandrs Aleksejevs, in Canada. Next, she completed two internships working with the ATLAS experiment, first with Prof. Michel Lefebvre and the Institute of Particle Physics at CERN and in Canada, and second with Dr. Reda Tafirout at TRIUMF in Canada. Inspired by her foundation in physics, her taste for adventure combined with her passion for the environment lead her to the Netherlands. She was awarded the Utrecht University excellence scholarship and began a Master's program in Meteorology, Physical Oceanography and Climate. In 2017, she obtained a Master's degree in Climate Physics (because they changed the name), completing a final project with Dr. Rupert Holzinger, applying mass spectrometry to semi-volatile organic compounds in the atmosphere atop the Cabauw tower. These experiences prepared her to pursue a PhD in the Physics of Fluids group at the University of Twente. Supervised by Dr. Richard Stevens, she studied atmospheric turbulence related to the performance of large-scale wind farms, as presented in this thesis. Besides research, she explored many new hobbies throughout her doctoral studies (and the pandemic), such as sword fighting, volley ball, and learning Dutch. 




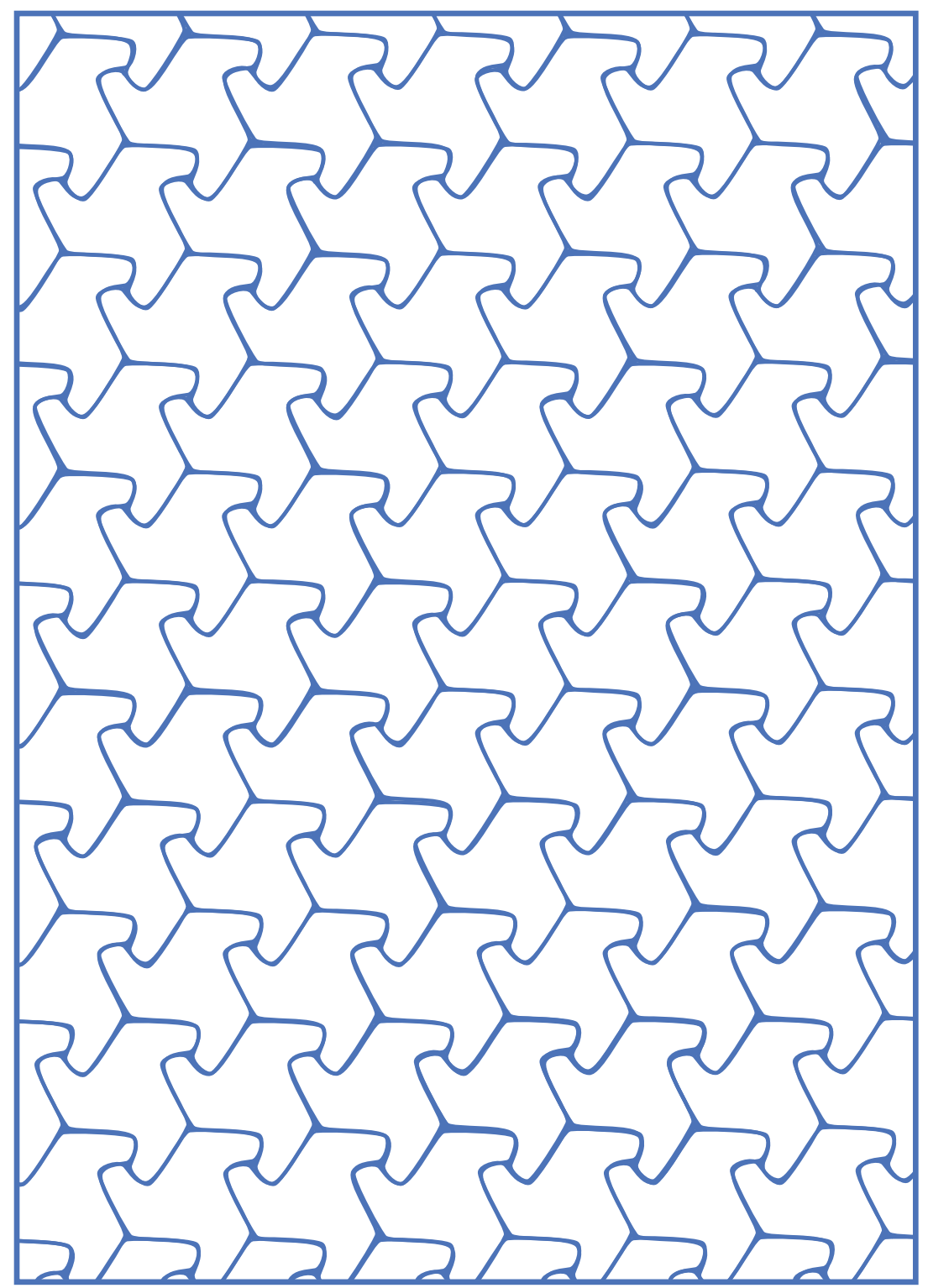


